\title{
Toroidal Effects on Drift Wave Turbulence
}

\author{
M.J. LeBrun, T. Tajima, M.G. Gray, G. Furnish, W. Horton \\ Institute for Fusion Studies \\ University of Texas at Austin \\ Austin, TX 78712
}

The universal drift instability and other drift instabilities driven by density and temperature gradients in a toroidal system are investigated in both linear and nonlinear regimes via particle simulation. Runs in toroidal and cylindrical geometry show dramatic differences in plasma behavior, primarily due to the toroidicity-induced coupling of rational surfaces through the poloidal mode number $m$. In the toroidal system studied, the eigenmodes are seen to possess (i) an elongated, nearly global radial extent (ii) a higher growth rate than in the corresponding cylindrical system, (iii) an eigenfrequency nearly constant with radius, (iv) a global temperature relaxation and enhancement of thermal heat conduction. Most importantly, the ineasured $\chi_{i}$ shows an increase with radius and an absolute value on the order of that observed in experiment. On the basis of our observations, we argue that the increase in $\chi_{i}$ with radius observed in experiment is caused by the global nature of heat convection in the presence of toroidicity-induced mode coupling. PACS: 52.65.tz, 52.25.Fi, 52.35.Kt

\section{DISCLAIMER}

\begin{abstract}
This report was prepared as an account of work sponsored by an agency of the United States Government. Neither the United States Government nor any agency thereof, nor any of their employees, makes any warranty, express or implied, or assumes any legal liability or responsibility for the accuracy, completeness, or usefulness of any information, apparatus, product, or process disclosed, or represents that its use would not infringe privately owned rights. Reference herein to any specific commercial product, process, or service by trade name, trademark, manufacturer, or otherwise does not necessarily constitute or imply its endorsement, recommendation, or favoring by the United States Government or any agency thereof. The views and opinions of authors expressed herein do not necessarily state or reflect those of the United States Government or any agency thereof.
\end{abstract}




\section{Introduction}

Drift wave instabilities and their subsequent turbulence are believed to be an important component of the experimentally observed anomalous transport $t^{1,2}$ of heat in tokamak plasmas. In this work we present our study of electron drift wave turbulence and ion temperature gradient driven drift wave turbulence through the particle simulation technique. We focus on the effect of toroidicity on these instabilities, the associated turbulence, and an particle and energy transport.

A nomuniform plasma in a shear-free magnetic field is unstable to an electron drift wave with a growth rate proportional to $(d n / d x)^{2}$ from either collisions or the electron Landau response. Due to the dependence on the square of the density gradient the mode is called the universal mode ${ }^{3}$ although there are mechanisms for stabilizing the instability.

The presence of shear, however, fundamentally alters the character of the mode, and necessitates a nonlocal treatment. ${ }^{4}$ The resulting differential eigenmode equation gives rise to a family of normal-mode solutions $\left(\phi_{l}(x), l=0,1,2, \ldots\right)$, localized to the rational surface $x=0$ where $k_{\|}=0$. Inclusion of the non-resonant kinetic electron effects near the mode rational surface in a sheared slab is found to make the drift wave absolutely stable for any finite amount of shear. ${ }^{5,6}$ The non-resonant electron stabilization arises from the region where $\left|k_{\|}\right|<\omega / v_{e}$, which is expected to be strongly influenced by both toroidicity and turbulent electron diffusion.

One important destabilizing mechanism for the drift wave is the effect of toroidal geometry, including poloidal mode coupling and trapped particles. In a toroidal system, the variation of magnetic field quantities in the poloidal direction $(\theta)$, as well as the magnetic drifts of the particles, can strongly couple the poloidal harmonics. Thus, drift waves at different mode rational surfaces will also be coupled. Using a simplified model, Taylor ${ }^{7}$ examined this case and found that linear toroidal mode coupling could eliminate the shear ion damping of the drift wave. An improved analysis of this problem by various 
researchers ${ }^{8-10}$ confirmed the existence of a marginally stable mode in the toroidal system. This new drift-wave branch is known as the "toroidicity-induced" mode, and inclusion of additional destabilizing effects have shown it to be absolutely unstable for small values of $k_{\perp} p_{i}(-10.3)$ and values of shear relevant to tokamaks. ${ }^{11-14}$

In addition to the electron drive associated with the universal mode, there are the driving mechanisms of the electron and ion temperature gradients and the unfavorable magnetic curvature on the outside of the torus. In fact recent experiments ${ }^{15,16}$ which raised the temperature gradients with auxiliary heating report enhanced heat transport over that without auxiliary heating. The destabilization of the ion drift wave by the combination of the inn temperature giadient and the infavorable magnetic curvature in the torus was predicted by Horton et al. ${ }^{17}$

While the aforementioned works have provided much information and insight as to the behavior of density and temperature gradient driven modes, they generally suffer several drawbacks. Due to the complexity of the analysis, many simplifying essumptions are often made and application to realistic systems may be difficult. In addition, nonlinear study is needed. Theory illustrates how dramatically toroidicity can alter the properties of drift wave instabilities and turbulence. Toroidal effects may likewise prove crucial for adequate understanding of the drift wave turbulence in tokamak plasmas and their transport properties. We should find that not only the electron drift wave but also other drift wave modes and the associated levels of transport are significantly affected by toroidal effects.

In the following section we describe the Toroidal Particle Code (TPC) simulation program. In Section III we study the results of universal mode turbulence simulation runs employing toroidal and cylindrical topologies. These runs employ a single high toroidal mode number and $\eta_{i}=\eta_{e}=0$. In Section IV, these results are extended to the ion tem. perature profile case, where we present runs with several values of $\eta_{i}$ using an adiabatic electron response. For $\eta_{i}=\eta_{e}=1$, comparisons between runs with kinetic and adiabatic 
electrons are made. Additional discussion and conclusions are given in Section $V$. 


\section{Toroidal Particle Code (TPC)}

\section{A. Computational Methods}

The Toroidal Particle Code, or TPC, is a multi-geometry, multi-model (primarily kinetic), and multi-platform simulation code developed at the Institute for Fusion Studies (IFS). ${ }^{18}$ TPC exceeds 60,000 lines and is written in MPPL (A More Productive Programming Language), a public domain Fortran preprocessor. A modular and hierarchical design is used, allowing the code to support a variety of dynamics types and/or field solvers, switchable via MPPL macro variables and conditional compilation.

A toroidal $(r, \theta, \zeta)$ metric is employed. Typically a global radial discretization $(r=0$ to $r=a)$ is employed, with a nonuniform grid spacing, although local $\left(r=r_{\min }\right.$ to $\left.r=r_{\max }\right)$ discretizations are also supported. The field representation is two or three-dimensional, where either a grid or mode expansion can be used to represent the azimuthal coordinate. The code is capable of operating in either the cylindrical or slab limit, which is used to assess the effects of toroidicity in a controlled fashion. Simulation runs are analyzed using the Tornidal Post Processor (TPP) which is the post run analysis program for TPC generated nutput. The post-processor includes a variety of independent diagnostic packages, including diagnostics for: spectral analysis and display, time evolution of potential, snapsloot diagnostics (density, temperature, etc.), transport analysis (e.g. thermal diffusivity as a function of radius or time), test particle trajectories, and interferogram analysis, among others.

The particle species in TPC are represented by a variety of descriptions, including full kinetic, drift kinetic, gyrokinetic, Boltzmann (adiabatic). Each of these can be modeled 1using standard particle-in-cell (PIC) treatment or a Lagrangian Vlasov (or $\delta f$ ) representation, and there is an optional Monte Carlo collision operator. At the time of this writing, TPC is being tested and used with the gyrokinetic and Lagrangian Vlasov models. For this paper we focus on results employing more conventional particle-in-cell models. That is, 
the inn species is advanced with the full Lorentz force, and the electron species is either drift kinetic or adiabatic. Additional details of the various algorithms employed by TPC are available elsewhere. ${ }^{18}$

Nithough the conventional full dynamics PIC model is known to suffer from inherent noise problems, the method is robust and free of some of the uncertainties of more modern approaches. Further, while noise may mask the growth of weak instabilities, it is not necessarily the dominant factor in the underlying transport, and has little effect on the real part of the frequency. A method, albeit an expensive one, for discovering limits to the simulation's validity is to increase the number of particles to see if the result changes, and this has been done for some cases in this work. For the rnajority of simulation runs presented in this work, the intrinsic noise is seen to be far overshadowed by the strength of the plasma instability (cf. Section IV).

\section{B. Geometry and field representation}

The geometry we employ is illustrated in Figure 1. The $(r, \theta, \phi)$ coordinate system we use is orthogonal and right-handed, with the surfaces of constant $r$ being nested, concentric circles of revolution (tori). The internal representation of the coordinates is such that the cole may run in the cylindrical limit simply by letting the toroidal curvature $(1 / R)$ go to zero, and the slab limit is obtained by letting the cylindrical curvature $(1 / r)$ go to zero. The metric components for this system are $h_{r}=1, h_{x}=r / r_{0}$, and $h_{\zeta}=R / R_{0}$, where $R=R_{0}+r \cos \theta, \zeta=-R_{0} \phi$, and the differential arc length is $d s^{2}=d r^{2}+r^{2} d \theta^{2}+R^{2} d \phi^{2}=$ $d r^{2}+h_{\chi}^{2} d \chi^{2}+h_{\zeta}^{2} d \zeta^{2}$.

For the runs discussed in this work the magnetic axis lies at $r=0$, and flux surfaces lie onl surfaces of constant $r$, corresponding to an idealized zero beta tokamak. Tokamak-like magnetic fields are used with

$$
\begin{aligned}
& B_{\phi}=B_{\phi_{0}} \frac{R_{0}}{R} \\
& B_{\theta}=\frac{r}{R_{0} q(r)} B_{\phi},
\end{aligned}
$$


where $q(r)$ is the safety factor, varying from $q(0) \simeq 0.6$ to $q(a) \simeq 4.0$ in the present studies. Electrostatic field quantities $(\rho, \Phi, \mathbf{E})$ are discretized using a nonuniform grid in $r$, a uniform grid in $\theta$, and a mode expansion (i.e. gridless) representation in $\zeta$. The nonuniform radial grid allows improved resolution near the rational surfaces, while being efficient and straightforward to implement when radial finite differences are used in the field solver. ${ }^{18}$ In $\theta$, field quantities are transformed between real and mode space as necessary using a Fast Fourier transform. The gridless representation in $\zeta$ allows a high degree of accuracy (and hence lower noise) in simulation of a single "high" toroidal mode number. A full three dimensional (3-d) grid representation is also available but not used in the runs described here. An example of the $(r, \theta)$ grid showing a typical nonuniform radial grid is given in Figure 2.

Note that in our system, a mode of the form $f(r) \exp (i m \theta-i n \phi)$ has a direct representation, in contrast to models which employ a square grid cross-section. This representation allows simple control of the cross-field resolution, a predictable fall off in mode strength with increasing mode number $m$ from the finite size particle effect (i.e. filtering), and a good match between the simulation quantities and the diagnostic package.

\section{Field Solver}

TPC solves the Poisson equation

$$
\nabla^{2} \Phi=-4 \pi \rho
$$

and computes the electric field $\mathbf{E}=-\nabla \Phi$ with the Laplacian decomposed into the two components given by

$$
\nabla^{2}=\nabla_{c}^{2}+\mathcal{C}
$$

where $\Gamma_{c}^{2}$ and $\mathcal{C}$ are defined by

$$
\begin{aligned}
\Gamma_{\mathrm{c}}^{2} & =\frac{\partial^{2}}{\partial r^{2}}+\frac{1}{r} \frac{\partial}{\partial r}+\frac{1}{r^{2}} \frac{\partial^{2}}{\partial \theta^{2}}+\frac{\partial^{2}}{\partial \zeta^{2}} \\
\mathcal{C} & =\frac{\cos \theta}{R} \frac{\partial}{\partial r}-\frac{\sin \theta}{R} \frac{1}{r} \frac{\partial}{\partial \theta}+\frac{R_{0}^{2}-R^{2}}{R^{2}} \frac{\partial^{2}}{\partial \zeta^{2}} .
\end{aligned}
$$


Here $\Gamma_{c}^{2}$ is the cylindrical Laplacian, and $\mathcal{C}$ represents the toroidal contribution $(\zeta \equiv$ $\left.-R_{1,} \phi\right)$. Eq. (3) is solved by Fourier analysis in $\theta$ and $\zeta$, finite differencing in $r$, and iteratively solving the resulting tridiagonal system of equations. Convergence scales with inverse aspect ratio, with less than 10 iterations typically required for 5 decimal places accuracy. The charge density is given by $\rho=\rho^{\prime} / J$ where $\rho^{\prime}$ is the flat-space charge density and $J=\partial(r, \chi, \zeta) / \partial(x, y, z)$ is the transformation Jacobian for the toroidal coordinate system (which also includes the effect of the nonuniform radial grid).

For an axisymmetric tokamak the Hamiltonian of the system is independent of $\zeta$ (or p). The Fourier modes $(n)$ in this direction are thus independent; $n$ is the so-called good quantum number of linear theory. On the other hand, the symmetry in $\theta$ is broken due to the effect of toroidicity, leading to a linear coupling between poloidal $(m)$ modes with a conpling coefficient on the order of the inverse aspect ratio $(\epsilon=0.2$ at $r / a=0.5$ in the runs reported here). Coupling between different $n$ modes occurs only nonlinearly such as through mode-mode coupling and with an effective coefficient of $e \Phi / T \sim 0.01-0.05$, which is much weaker than the $m$ coupling.

Therefore for the runs performed in this work we choose a gridless representation for the fields in $\zeta$, with a single toroidal $n$ mode retained. Additional toroidal modes may be arded in a measured fashion (to be examined in a later work). This approach makes for a good lowest-order calculation, in which the effects of the strong $\theta$ coupling are accurately modelled while retaining good radial resolution around the rational surfaces. Further, this situation is directly relevant to the case of a plasma with a limited set of dominant toroidal $n$ modes such as might occur near marginal stability.

In the limited-n configuration the simulation runs are quieter than for a full $3-d$ run, all clse being equal, which compensates somewhat for the intrinsic noise of a full dynamics model for the ions. Retaining only a single or limited set of toroidal modes allows a distance of several radial grid points to separate adjacent rational surfaces. Nonlinearities such as the advective nonlinearity are retained through following the exact (or nearly 
exact) particle dynamics.

\section{Particle Dynamics}

In the standard case the ion motion is given by the Lorentz equations of motion

$$
\begin{aligned}
& \frac{d \mathbf{x}}{d t}=\mathbf{v} \\
& \frac{d \mathbf{v}}{d t}=\frac{q}{m}(\mathbf{E}+\mathbf{v} \times \mathbf{B})
\end{aligned}
$$

which are advanced in time using a non-dissipative leap frog algorithm. ${ }^{18}$

The electron motion is given by the drift equations

$$
\begin{aligned}
\frac{d \mathbf{x}}{d t} & =\mathrm{v}_{d}+v_{\|} \mathbf{b} \\
\mathrm{v}_{d} & =\mathrm{u}_{E}+\frac{\mathbf{b}}{\Omega_{e}}<\left\{(\mu / m) \nabla B+v_{\|}^{2}(\mathbf{b} \cdot \nabla \mathbf{b})\right\} \\
\frac{d v_{\|}}{d t} & =-\frac{e}{m} E_{\|}-\frac{\mu}{m} \mathbf{b} \cdot \nabla B
\end{aligned}
$$

where $\mathbf{u}_{E}=\mathbf{E} \times \mathbf{b} / B, \mathbf{b}=\mathbf{B} / B, \mu=\frac{1}{2} m v_{\perp}^{2} / B$, and $\Omega_{e}=-e B / m c$. Eqs. $(9-11)$ are advanced in time via a predictor-corrector algorithm.

\section{E. Diagnostics}

Most of the diagnostics used by TPC are fairly standard and will not be discussed here. An exception is the transport analyzer, which is used extensively in analysis of the simulation runs in Section IV. The diffusivity is traditionally obtained macroscopically, by dividing the particle flux by the (negative) gradient of the density profile. Similarly, the thermal diffusivity is traditionally obtained by dividing the energy flux by the product of the mass density and the (negative) gradient of the temperature profile. The problem with either of these methods is that the density and/or temperature gradient may become arbitrarily small in a given radial region, resulting in an extremely noisy measurement for $D$ and $\chi$. This can be solved most efficiently by using a microscopic means of calculation, based on the statistical mechanics of a random-walk process. Namely, the diffusivity can 
be written as

$$
D=\frac{\sum_{j=1}^{N}\left[x_{j}(t)-x_{j}(0)\right]^{2}}{2 t N}
$$

fur a sample of $\mathrm{N}$ particles (indexed by $j$ ) in a given region of space, and $x$ is the cross field coordinate. The thermal diffusivity can be written in a similar manner by

$$
x=\frac{\sum_{j=1}^{N}\left[T_{j}(t) x_{j}(t)-T_{j}(0) x_{j}(0)\right]^{2}}{2 t N T^{2}}
$$

where $T$, is the kinetic energy for the $j$ th particle and $T$ is the bulk kinetic energy. Instead of $t=0$, an appropriately chosen initial time or a sliding window may be used. This formula is clearly capable of representing both conductive and convective heat transport, and test runs of convective transport show good agreement between the microscopic and inacroscopic methods of calculation. Details of these tests will appear in a separate work. For the simulation runs reported here, a sample of $N=2048$ or $N=8192$ particles for transport statistics was used. This is sufficient to obtain transport coefficients as a function of both $r$ and $t$ from the microscopic formulae, although the decrease in the number of particles in the tail causes poorer statistics there (the transport markers are sampled from the particles making up the bulk plasma, and thus have the same density and temperature profiles). In addition the data at smaller radius $(r / a \lesssim 0.1)$ is not as reliable for the same reason, as the vanishing of the Jacobian near $r=0$ leads to a similar decrease in the number of particles for a given value of density there. Thus, plots of transport coefficients presented in Section IV typically range from $0.1 \leqq r / a \leqq 0.5$ only. :

In this work we express $\chi$ in units $\rho_{i}^{2} v_{i} / L_{n}$, whereas the natural units for the postprocessor are $\rho_{i 0}^{2} \Omega_{i}$, with $\rho_{i 0}$ the mean value of $\rho_{i}$ across the entire plasma. The conversion between the two systems of units raises the question of which "average" value to use for the ion Larmor radius. Any average value that ignores the localization of the mode will lo incorrect, since it is not representative of the value felt by the physical conductive or cnnvective process. Since $\chi$ is thought to scale with some power of $\rho_{i}$, it makes the most physical sense to average only over the active region when calculating $\rho_{i}$. This scaling 
gives a conversion factor of $\left(\rho_{i 0}^{2} / \rho_{i}^{2}\right)\left(L_{n} / \rho_{i}\right)=23.2$, where we have taken the center of the active region to be at $q(r)=1$ (note that for the cases studied, the central mode is $m=9$, $n=9$, and is close to the location of maximum power). 


\section{Universal Mode Turbulence}

In this section we compare theory and simulation results for the universal drift wave instability and the associated turbulence in slab, cylindrical, and toroidal geometries.

\section{A. Linear Theory and Simulation in a Slab Plasma}

In nrder to describe the linear eigenmode structure and the stability of the universal mode in a sheared slab plasma, we need to compute the perturbed electron and ion densities and invoke quasineutrality. ${ }^{19}$ The electron density response $\bar{n}_{e}$ may be written ${ }^{5}$ as

$$
\tilde{n}_{e}=\frac{e n_{0}}{T_{e}}\left[1+\zeta_{e} Z\left(\zeta_{e}\right)\left(1-\frac{\omega_{e}^{*}}{\omega}\right)\right] \dot{\Phi}
$$

and the ion response $\bar{n}_{i}$ as

$$
\bar{n}_{i}=-\frac{e n_{0}}{T_{i}}\left[1+\zeta_{i} Z\left(\zeta_{i}\right)\left(1-\frac{\omega_{i}^{*}}{\omega}\right) \Gamma_{0}\left(b_{i}\right)-\zeta_{i} Z\left(\zeta_{i}\right)\left(1-\frac{\omega_{i}^{*}}{\omega}\right) \frac{d \Gamma_{0}}{d \dot{b}} \rho_{i}^{2} \frac{\partial^{2}}{\partial x^{2}}\right] \hat{\Phi}
$$

where $n_{n}$ is the average local plasma density, $T_{j}$ the temperature of the $j$ th species $(j=e$ or $i$ ), and where $\zeta_{j}=\omega / \sqrt{2}\left|k_{\|}\right| v_{j}, v_{j}=\left(T_{j} / m_{j}\right)^{1 / 2}, b_{i}=k_{\perp}^{2} \rho_{i}^{2}, \rho_{i}=v_{i} / \Omega_{i}, \rho_{0}^{2}=\tau \rho_{i}^{2}$, $T=T_{e} / T_{i}, \Gamma_{0}\left(b_{i}\right)=I_{0}\left(b_{i}\right) \exp \left(-b_{i}\right), I_{0}$ is the modified Bessel function, and $Z$ is the plasma dispersion function. The diamagnetic frequencies can be written in a convenient dimensionless form as

$$
\frac{\omega_{e}^{*}}{\Omega_{i}}=-\tau \frac{\omega_{i}^{*}}{\Omega_{i}}=\left(\kappa \rho_{0}\right)\left(k_{\perp} \rho_{0}\right)
$$

where $\kappa$ is the inverse density scale length. By imposing the quasineutrality condition $\bar{n}_{g} \approx \bar{n}_{i}$, we derive the eigenmode equation which takes on the form of a differential equation in the radial direction ( $r$ or the local radial coordinate $x$ ):

$$
\left[\rho_{a}^{2} \frac{d^{2}}{d x^{2}}+Q(x, \omega)\right] \hat{\Phi}=0
$$

where

$$
\begin{aligned}
Q= & {\left[-\zeta_{i} Z\left(\zeta_{i}\right)\left(1-\frac{\omega_{i}^{*}}{\omega}\right) \frac{d \Gamma_{0}}{d b}\right]^{-1} } \\
& \left\{\left[1+\zeta_{e} Z\left(\zeta_{e}\right)\left(1-\frac{\omega_{e}^{*}}{\omega}\right)\right]+\tau\left[1+\zeta_{i} Z\left(\zeta_{i}\right)\left(1-\frac{\omega_{i}^{*}}{\omega}\right) \Gamma_{0}\left(b_{i}\right)\right]\right\}
\end{aligned}
$$


This equation is solved subject to the appropriate boundary conditions, that is, outgoing waves for $x= \pm \infty$. For large $|x|$ the solutions of Eq. 17 are given by the Wentzel, Kramers and Brillouin (WKB) condition

$$
\dot{\Phi}(x) \sim Q^{-1 / 4}(x) \exp \left\{ \pm i \int^{x} \frac{d x^{\prime}}{\rho_{0}}\left[Q\left(x^{\prime}\right)\right]^{1 / 2}\right\}
$$

where the outgoing wave boundary condition requires that for $\operatorname{Im} \omega>0$ the choice of $\pm i$ is such hat $|\Phi(x)| \rightarrow 0$ for $|x| \rightarrow \infty$. Since this formulation is symmetric about the mode rational surface $(x=0)$, the equation may be solved in the half-space between $x=\|$ and $x \rightarrow+\infty$, with the specification of even or odd parity about $x=0$ for the additional boundary condition. The method of numerical integration for determining the eigenfunction and eigenvalues from Eq. 17 and the outgoing wave boundary conditions is given by Sydora et al. ${ }^{20}$

Simulation of the slab drift wave was performed using the two-dimensional slab limit of TPC, obtained by setting $h_{x}=1$ and $h_{\zeta}=1$. A weakly nonuniform grid in $x$ was employed to improve the resolution about the mode rational surface. An exponential density profile of the form $n(x) \sim n_{0} \exp (-\kappa x)$ was used, with $\kappa=|\partial \ln n / \partial x|=L_{n}^{-1}$ the constant inverse density scale length.

The remaining simulation parameters are given by : $N_{p}=49152$ (the number of particles in each species), $L_{x}=L_{y}=64 \Delta_{0}\left(\Delta_{0}\right.$ is the average grid spacing in $\left.x\right), N_{\bullet}=$ $N_{y}=64, \Delta t=2.0 \omega_{e}^{-1}, v_{t h}=2.5 \Delta_{0} \omega_{e}, T_{e} / T_{i}=1, m_{e} / m_{i}=0.01, \Omega_{e} / \omega_{e}=10$, and particle "size" of a grid spacing in both $x$ and $y$ (using two passes of the binomial digital filter in $\left.x^{18}\right)$. The shear and densily scale lengths are given by $L_{a} / L_{n}=13.6, \kappa \rho_{i}=0.17$, with $\rho_{i} / \Delta_{0}=2.5$. The mode rational surface is at $x=x_{0}$, where $x_{0}=L_{x} / 2$ and $x$ ranges from 0 to $L_{x}$. The condition that the ion resonance layers remain within the system is given by the condition that $\left|x_{i}-x_{0}\right| \leq L_{x} / 2$, where $x_{i}$ is given by the resonance condition $\omega=k_{\|}\left(x_{i}\right) v_{i}$. Since the drift wave frequency is smaller than the diamagnetic frequency, 
this will be satisfied if $\omega^{*}=k_{\|}\left(x_{i}\right) v_{i}$. Thus, we have

$$
\begin{aligned}
\left|x_{i}(\omega)\right|<\left|x_{i}\left(\omega^{*}\right)\right| & =\frac{\omega^{*} L_{s}}{k_{y} v_{i}} \\
& =\rho_{i} \frac{L_{\dot{\theta}}}{L_{n}}
\end{aligned}
$$

where $k_{\|}=k_{y} x / L_{s}$. For the present parameters, $x_{i}(\omega)$ is just inside the simulation region for the lowest mode $\left(k_{y} \rho_{s}=0.24\right)$, and well inside for higher mode numbers.

The energy for the run was conserved to within $0.3 \%$ for 6000 time steps. The first five modes (in $y)$ of the potential $\Phi\left(x, k_{y}\right)$ are stored for analysis. For $k_{y}=2 \pi m / L_{y}$, with $m=1, \ldots, 5$, we have

$$
k_{y} \rho_{\mathrm{i}}=0.24,0.49,0.74,0.98,1.23
$$

for the five modes examined.

As $k_{y} \rho_{i}$ is increased, the frequency decreases due to finite ion Larmor effects. This reduction in frequency in turn causes the eigenfunction to become increasingly localized since the ion resonance moves closer to the rational surface. Both effects are seen in the simulation. The theoretical and observed frequencies for the $m=3$ and $m=5$ modes agree within the accuracy of the spectral estimator, and frequencies for the remainder agree within a factor of two. The greatest difference between the observed eigenfunctions and eigenvalues occurred for the lowest mode number, $m=1$; this is most likely caused by interaction of the mode with the boundary. As illustration we show the measured eigenmode structure for the $m=2\left(k_{y} \rho_{i}=0.49\right)$ mode shown in Figure $3(\mathrm{~s})$ and the theoretical result in Figure 3(b).

\section{B. Theory of Drift Waves in Toroidal Geometry}

The shear stabilization of the drift wave in the slab plasma as discussed in the introluction is fundamentally altered by the spatial inhomogeneities, i.e. the breakdown of symmetry brought about by toroidal geometry (measured by $r / R$ ). In a toroidal configuration with realistic $r / R$ the magnetic fields have a strong variation with the poloidal 
conrdinate $\theta$, and the Laplacian operator itself has $\theta$-dependent terms. This $\theta$ dependence results in coupling between drift wave eigenmodes at neighboring mode rati nal surfaces, which can inhibit or increase the convection of energy. Since it is this convection that is primarily responsible for the stabilization of the mode in the slab model, the stability and mode structure properties of the drift wave are substantially modified.

The model employed by Taylor ${ }^{7}$ was that of a plane slab, in winch the magnetic field strength or shear varied strongly in the "poloidal" direction (represented here by the $y$ coordinate). This variation coupled the poloidal Fourier components, resulting in a differential-difference equation in the $(x, y)$ coordinates. The electron response was assumed to be adiabatic, and a fluid ion response was used. The assumption of weak dependence on poloidal mode number $(m)$ allowed $m$ to be approximated as a continuous quantity. This "strong-coupling" approximation reduced the two dimensional partiai differential equation to an ordinary differential equation, expressed as a combination of both $x$ and $m$. The main conclusion of Taylor's work is that shear damping normally associated with the drift wave is severely reduced, resulting in a mode that is radially extended over an appreciable length of the plasma. By contrast, the slab-like drift mode is very localized around the mode rational surface.

The nullification of shear damping in a toroidal system was subsequently explored by a number of researchers, using more realistic theoretical models. The radially extended nature of the mode was verified by Ross and Miner. ${ }^{21}$ The two-dimensional eigenmode. equation in a toroidal configuration was considered by Horton et al, ${ }^{22}$ who obtained silutions in both the weak-coupling and strong-coupling limits. Subsequent research efforts ${ }^{10,8,8,23,11,24}$ have typically employed the ballooning mode representation to express perturbed quantities, in order to rigorously satisfy periodicity in both $\theta$ and $\phi$. With this approach, the validity of the solutions formally span the range from weak to strong coupling. The majority of the research efforts, however, have not pursued a fully kinetic treatment (represented in the nonadiabatic particle response), nor solved the higher order 
radial problem.

The published literature on the toroidal drift wave problem shows considerable variation in the approximation used for reducing the particle response, which is a consequence of the complexity of these terms in toroidal geometry. Even when the trapped particle motion is taken into account a small $r / R$ approximation $\left(B=B_{0}(1-r / R \cos \theta)\right)$ is used to shtain the elliptic integrals $E(m), K(m)$, with $m=v_{\|}^{2} / \epsilon v_{\perp}^{2}$. Adiabatic electrons and fluid inns are often employed, perhaps with the addition of a destabilizing trapped electron response term. This approach is sufficient for investigating the qualitative character of the inode, including its radially-extended ballooning character. However, the importance of the full kinetic effect on the stability is hard to gauge. A formal expression of the full particle response was given in 1980 by Connor et al, ${ }^{25}$ but the expressions must undergo considerable approximation before an actual solution is possible. If nonadiabatic terms are kept, the manner of solution will necessarily employ either an integral formulation or an iterative method, which makes theoretical analysis much more difficult.

The nonadiabatic electron passing response was included perturbatively by Chen and Cheng ${ }^{9}$ and using an integral method by Hesketh. ${ }^{24}$ Each of these works employed kinetic ions (under the assumption $\omega \gg \omega_{D_{i}}$ ) and obtained qualitatively similar results. The growth rate was found to increase without bound as $k_{\perp} \rho_{i}$ is increased, as well as with increasing toroidicity (measured by the parameter $\epsilon_{n}=L_{n} / R_{0}$ ). For the parameters studied by Hesketh, the mode reached a maximum growth for a shear of $\hat{s} \sim 1.7$. The neglect of the ion drift resonances, however, is not justified at larger values of $k_{ \pm} \rho_{i}$, since the real frequency decreases as this parameter increases. The inclusion of this resonance proves to be an important stabilizing effect, as was shown by subsequent papers. ${ }^{12-14}$ The result is that the growth is maximized at a value of $k_{\perp} \rho_{i} \sim 0.3-0.4$. A more thorough thenretical treatment of the toroidal drift universal wave problem, including the nonadiabatic electron response and ion drift resonance, is found in a paper by Schep and lenema. ${ }^{14}$ This investigation was based on the earlier work of Schep et al, ${ }^{26}$ which con- 
sidered approximate forms for the integral trapped and passing electron responses. This work shows that when both response terms are kept, large cancellations may occur, and the total response gives the proper behavior when one passes to the infinite aspect ratio limit (plane slab). Using these methods in the toroidal drift wave problem, the real and imaginary frequency variation was found ${ }^{14}$ to be qualitatively similar to that predicted by Cheng and Tsang ${ }^{12}$ and by Hesketh. ${ }^{13}$ To expedite comparison of theoretical findings til the simulation results, we choose pararneters for the simulation run similar to those studied by Schep and Venema. ${ }^{14}$

An additional point discussed in the literature concerns the relation of the toroidicityinduced mode to the slab-like mode, as the toroidicity (i.e. $\epsilon_{n}$ ) is increased. In the work of Hastie et $a l^{10}$ and Choi and Harton, ${ }^{8}$ the slab mode was observed to evolve continuously into the toroidal mode, while Chen and Cheng ${ }^{\theta}$ argue that the mode is a completely different branch. The apparent conflict between these observations was resolved in a separate investigation by Schep and Venema, ${ }^{27}$ where it was shown that the slab mode always connects to one of the harmonics of the toroidicity-induced mode. The connection is usually one of the higher, more heavily damped harmonics, depending on parameters.

The appearance of multiple drift wave harmonics in toroidal geometry can be understood by analogy to the band structure in solid state physics. If the radial variation of the equilibrium quantities is sufficiently weak, the potential will be symmetric with respect to translation in the radial coordinate over an integral number of rational surfaces. This local translation symmetry admits solutions which are Bloch functions, resulting in a band structure for the eigenvalues known as the Brillouin effect. Similarly, the toroidal drift wave eigenmode equation admits a number of solutions in terms of multiple harmonics, resulting from the additional degrees of freedom in the presence of multiple mode rational surfaces. Although the fundamental harmonic will have the strongest growth, the higher harmonics may be unstable or only weakly damped as well. However, the higher order harmonics are usually not considered in the theory. Depending on parameters, the slab- 
like mode may connect to the lowest even harmonic (as observed in the earlier papers) or more usually, to a higher even harmonic. Parameter studies by Schep and Venema ${ }^{27}$ were presented in which the slab-like mode connected to the $n=6$ or $n=8$ toroidicity-induced mode.

The theoretical treatment of the toroidal drift wave problem is in principle similar to that of the slab problem. The particle distributions are assumed to satisfy the Vlasov equation, in which the lowest order distributions represent the equilibrium solutions. For an electrostatic perturbation, the perturbed distributions are obtained by a time integral along the unperturbed particle orbits of the electrostatic potential and zeroth order particle distribution functions. The perturbed densities are obtained from the velocity space integration of the perturbed particle distribution functions, and the quasineutrality condition gives an integral equation for the potential.

In the slab analysis, the integral equation for the potential is approximated as a differential equation of a single variable. In this case, the orbit integral of $\phi\left(x\left(t^{\prime}\right)\right)^{*}$ is evaluated using the Taylor expansion of the potential in $\left(x\left(t^{\prime}\right)-x\right)^{n}$, which is valid for sufficiently small radial wavenumbers $k_{x} \rho_{i} \ll 1$. For the toroidal problem, however, two points should be noted. First, the perturbed quantities must be a function of both $r$ and $\theta$ due to the poloidal inhomogeneities. This leads to a partial differential equation of two variables, which is required in order to describe the shift of the dominant poloidal mode number with a translation in radius. Second, an integral term will be necessary in the toroidal problem even for small $k_{x} \rho_{i}$ to retain the trapped particle responses. This integral term occurs due to the increased complexity of the particle motion, which cannot be given in a simple, closed form for finite $r / R$. The perturbed distribution functions can be obtained through adoption of gyrokinetic coordinates ${ }^{25}$; however, the resulting expressions are very complicated and do not exist in closed form. Although approximate forms of the perturbed distributions exist, ${ }^{25,26}$ the subsequent velocity space integrations required for the perturbed densities present serious additional difficulties. 
Here we consider for illustration a simplified theoretical model which retains many of the features of a more precise treatment. The fluid ion response is adopted, and an electron response of the form

$$
n_{e}=n_{0}\left(\frac{e \Phi}{T}\right)(1-i \delta)
$$

where $\delta$ is some simple anti-hermitian operator that governs the nonadiabatic electron response. The perturbed potential is written in the form

$$
\phi(\theta, \rho) \exp [i(N \zeta-M \theta)-i \omega t]
$$

where $\rho=r-r_{0}$ is the radial distance from the mode rational surface given by $M=$. $N q\left(r_{0}\right), q$ is the safety factor, and $N$ is the toroidal mode number. The toroidal dependence can be dropped by axisymmetry, and the resulting eigenvalue problem takes on the general form

$$
\hat{\mathcal{L}}(\theta, \rho ; \omega) \phi(\theta, \rho)=0
$$

The operator $\dot{\mathcal{L}}$ is in general an integral operator, which is represented by the $i \delta$ operator in this analysis. With some approximation, $\dot{\mathcal{L}}$ can be given by

$$
\hat{\mathcal{L}}=\frac{\partial^{2}}{\partial x^{2}}-\sigma^{2}\left(\frac{\partial}{\partial \theta}+i x\right)^{2}-\epsilon\left(\cos \theta+i \hat{s} \sin \theta \frac{\partial}{\partial x}\right)-\lambda
$$

where $k=n q / r, \hat{s}=r q^{\prime} / q, x=k \rho \hat{s}$, and the parameters $\sigma$ and $\epsilon$ are given by

$$
\sigma=\frac{\epsilon_{n}}{q \tau b \hat{s}}, \quad \epsilon=\frac{2 \epsilon_{n}}{\tau b \dot{s}^{2}}
$$

where $\epsilon_{n}$ is the toroidicity $\left(L_{n} / R_{0}\right)$ and $b=k^{2} \rho_{i}^{2}$. The eigenvalue $\lambda$ is related to the frequency by

$$
\lambda=\frac{\omega / \omega^{*}-1+b(1+\tau)-i \delta}{b \dot{s}^{2}(1+\tau)} .
$$

Vote that Connor and Taylor ${ }^{28}$ included an additional term, resulting from the variation if $\omega^{*}$ with radius. 
For the drift wave problem; the modes of interest have long parallel wavelengths and short perpendicular wavelengths. The requirement of long parallel wavelength is given as

$$
\mathbf{B} \cdot \nabla \Phi \approx 0 \text {. }
$$

This condition is well satisfied in the present analysis only near the rational surface, i.e. $q(r)=M / N$. Alternately, we can adopt an eikonal form of the perturbed potential as

$$
\Phi(r, \theta) \exp [i S(r, \theta, \zeta)]
$$

such that

$$
\mathbf{B} \cdot \nabla S(r, \theta, \zeta)=0
$$

This representation satisfies the long wavelength requirement, but the resulting functions $\Phi(r, \theta)$ and $S(r, \theta, \zeta)$ are periodic in theta only at a mode rational surface. The dual requirements of periodicity and long parallel wavelength can be satisfied through use of the ballooning mode representation for the perturbed potential. ${ }^{20,30}$ We then make the expansion

$$
\Phi(x, \theta)=\sum_{m=-\infty}^{+\infty} \exp (-i m \theta) \int_{-\infty}^{+\infty} d \dot{\theta} \dot{\Phi}(\hat{\theta}, x) \exp (i m \hat{\theta})
$$

where the dependent coordinate in the poloidal direction has become $\hat{\theta}=\theta+2 \pi n$ (the "extended" poloidal variable), and $\hat{\Phi}$ is given by

$$
\dot{\Phi}(\dot{\theta}, x)=A(x) \exp \left[-i x\left(\dot{\theta} \pm \theta_{k}\right)\right] f(\dot{\theta}, x) .
$$

Here $A(x)$ is a slowly varying envelope for the mode, and $f(\hat{\theta}, x)$ satisfies to lowest order a one-dimensional eigenmode equation in the extended variable $\hat{\theta}$ with a parametric dependence on $x\left(\theta_{k}\right.$ is a slowly varying function of radius, and is not specified to the lowest irder). The lowest order eigenmide equation in the toroidal model is given in terms of the extended poloidal variable $\theta$, in contrast to the radial variable $\rho$ in the slab model. This change occurs as a result of the poloidal inhomogeneities and give additional wave trapping terms in the one-dimensional mode equation. The radial envelope dependence 
in the present model can be obtained via a radial eigenmode equation to higher order in the expansion parameter $1 / N$.

With inclusion of the nonadiabatic response terms (and ion drift resonances), the equation for $f(\hat{\theta}, x)$ takes on the general form

$$
\left[\frac{d^{2}}{d \dot{\theta}^{2}}-V(\dot{\theta}, \omega)\right] \alpha(\dot{\theta}, \omega) \dot{\Phi}=\int d \dot{\theta}^{\prime} K\left(\dot{\theta}, \dot{\theta}^{\prime} ; \omega\right) \dot{\Phi}(\dot{\theta})
$$

(from Schep and Venema ${ }^{14}$ ). Their method of solution for Eq. (33) employs a shoot. ing code for solving the homogeneous equation by initially neglecting the integral term; this first order perturbed potential $\dot{\Phi}(\hat{\theta})$ is then numerically integrated to obtain the $\dot{\theta}$-dependent right hand side as part of an iteration procedure.

This theoretical analysis predicts the existence of unstable solutions. The most un: stable mode is the lowest harmonic $(l=0)$, and is the only harmonic considered. The ion drift resonance has a stabilizing influence on the electron drift mode, so that the perpendicular wavenumber of maximum growth occurs at about $k_{\perp} \rho_{i} \sim 0.2$, for system parameters of $\hat{\boldsymbol{s}}=q=\tau=1, \epsilon_{n}=0.1, m_{i} / m_{e}=1836$ and $\epsilon=0.1$ (inverse aspect ratio). For comparison purposes, these parameters were duplicated as closely as possible in the simulation, but numerical constraints prevent an exact match (most significantly in the mass ratio). Furthermore, the need to maintain adequate radial resolution ensures that the equilibrium quantities will vary significantly over the width of the mode. The quantities that vary in radius in the simulation include the shear $(\hat{\boldsymbol{j}})$, toroidicity $\left(\epsilon_{\boldsymbol{n}}\right)$, and diamagnetic frequency $\left(\omega^{*}\right)$. The variation of equilibrium quantities in the simulation may require the higher order equation for the radial dependence to be solved in order for close correspondence with theory. In addition, the theory is strictly valid only in the limit of high toroidal mode number; the relative smallness of the toroidal mode number in the simulation $(n=9)$ may require calculation to higher order.

The effect of varying the diarragnetic frequency has been examined by Connor and Taylor, ${ }^{28}$ where it is shown thri a decrease in the strength of coupling between modes at neighboring mode rational surfaces will result. This coupling crucially determines the 
qualitative nature of the mode. For very weak coupling, the Fourier modes at neighboring mode rational surfaces are nearly independent and are radially localized, as in the slab limit. These modes possess weak "sidebands" on neighboring mode rational surfaces, which becone stronger as the coupling is increased. For sufficiently strong coupling, the Fourier modes sum to become a single "quasimode", which extends over many mode rational surfaces. The quasimode tends to be localized in $\theta$, so that it may appear to "balloon" towards the outside of the torus. The parameter regime considered by the simulation does produce the theoretically predicted ballooning behavior, although effects due to the radial variation of $\omega^{*}$ may also be present.

\section{Toroidal Simulation}

Here we present simulations for $\eta_{i, e}=0$ in a toroidal and cylindrical configuration using a single toroidal mode number, $n=9$. The rational surfaces contained within the system range from $q=5 / 9(m=5)$ to $q=15 / 9(m=15)$. Undesired rational surfaces are excluded by eliminating the appropriate Fourier components of the electric potential. For these runs the slab-like drift ion resonance layers for each rational surface are contained within the simulation region, satisfying the boundary conditions necessary for stabilization of the slab-like drift wave eigenmodes. For reference, we summarize all the runs performed in this work in Table 1, along with those modes expected to contribute to the fluctuations and transport.

The parameters for the simulation run are as given in Table 2 (note: for this run nnly, the maximum poloidal mode number is 15 ). The radial variation of the initial electron density, the variation of grid spacing in $r$, and the number of particles per cell as a function of cell number is shown in Figure 4. The marks above the nonuniform grid ticks indicate the locations of the mode rational surfaces. See Figure 5 (a) for the radial variation of the safety factor $q$, shear parameter $\dot{\boldsymbol{j}}$, and the toroidicity parameter $\epsilon_{n}$. The electron/ion profiles have been loaded in such a way as to minimize the departure from 
quasineutrality. The simulation was run for a total of 15,000 time-steps $\left(T=6000 \Omega_{i}^{-1}\right)$. Energy conservation was $15 \%$ over the length of the run. The gradual increase in energy is caused by inaccuracy in the time integration due to undersampling with respect to the highest frequency waves, and this slow increase in the thermal energy can be ignored (it is stochastic in nature and does not affect the results presented here).

The toroidal run shows instability in the vicinity of the $m=7-9$ rational surfaces $(r a \sim 0.4)$ with growth rate $\gamma: \omega^{*} \sim 0.12$, close to the theoretical value when trapped electruns are taken into account. ${ }^{31}$ This was accompanied by weak profile flattening in the same radial region, which diminished after saturation of tile mode. The growth rate as measured hy the simulation run is higher than the value given by Schep and Venema ${ }^{14}$ due th a combination of effects: presence of trapped electrons, a larger inverse aspect ratio, and a larger mass ratio $\left(m_{e} / m_{i}=0.01\right)$ than in that work (note: all of these effects are destabilizing). Saturation for these modes occurs at a level of $|e \Phi / T| \sim 0.04$. Recall that $k_{\theta} \rho_{i} \sim 0.2$, and using $\rho_{i} / L_{n} \sim 0.025$, we have $1 / k_{\theta} L_{n}$ for the observed modes (see Fig. 5). By contrast, the cylindrical run showed no discernible unstable modes and profile modification.

We next examine the mode structure of the potential on long time scales via spectral analysis, with the power spectrum is calculated by the maximum entropy method. This technique employs a frequency-space approximation that is well suited for resolving sharp spectral features. ${ }^{32}$ The observed frequency spectrum and its dependence on radius and mode number has new noteworthy features.

In Figure $6(\mathrm{a})$ is the spatially averaged spectral density as a function of frequency. Note the rather broad spectrum similar to that observed in laboratory tokamak plasmas, although the peaks from the linear mode spectrum are still apparent. The broad spectral rngion decays as $S(\omega) \sim \omega^{-1}$ for $\omega \ddot{z} \omega^{*}$

In Figure 6 (b) we plot maximum power as a function of $\omega / \Omega_{i}$ for the $m=7$ mode, and in Figure $b(c)$ we plot $\omega / \Omega_{i}$ as a function of radius for the $m=7$ through $m=11$ poloidal 
modes (the arrows mark the rational surfaces). This diagnostic eliminates the spectral widths in order to see the linear physics aspects more clearly-peaks in the calculated spectrum are located numerically for each radial position and mode number, with only the strongest power peaks retained.

It is clear from Figure 6 (b), that there exists a multiplicity of modes with frequencies below the diamagnetic frequency. This result is a consequence of the sirong coupling of the poloidal modes in toroidal geometry, which allows multiple toroidal drift harmonics to appear. Further, for an individual $m$ value, this may be manifested in a nearly $\delta$-function dependence of the maximum power on frequency (each peak has a finite width, however). Plots for nther values of $m$ are surprisingly similar for the highest power peaks, though. splitting often occurs at lower power

In Figure 6 (c), we see a global preference for certain discrete frequencies, with mode activity concentrated in "bands" in frequency space. This is an expected result for poloidal modes strongly coupled by the toroidicity. Some variation of the dominant frequency between different mode numbers occurs due to nonlinear splitting and radial variation of the equilibrium. Note that the theory eliminates the radial variation through the assumption of translational invariance, and is thus only partly applicable to the situation in a realistic plasma, even when nonlinearities are mild.

The lowest two frequency bands show the highest degree of overlap between modes with different poloidal wave number $m$, and are by far the strongest modes. The lowest frequency band occurred at roughly $\omega / \omega^{*}=0.4$, in good agreement with theory. ${ }^{14}$ The cylindrical run, on the other hand, shows no band structure in its frequency response, as expected. In addition, the lowest harmonic seen in the cylindrical run has a frequency approximately that of the second harmonic in the toroidal run; the ultra-low frequency harmonic appears only in the toroidal case. The ion magnetic drift frequency is on the order of $\dot{\omega}_{D_{i}} / \Omega_{i} \sim 0.001$ at $k_{\theta} \rho_{i}=0.2$, so the ion magnetic drift resonance may significantly affect the lowest harmonic. The modes are seen to propagate in the direction of the 
electron diamagnetic drift.

Nost modes extend over a large number of mode rational surfaces, but still show some radial localization. This is consistent with the physics of the parameter regime. For this parameter regime, the diamagnetic frequency for a given poloidal mode is constant with radius, varying only with $m$. However, the diamagnetic frequency for the mode ensemble increases as $n q(r)$. It is this ensemble value that determines the overall appearance and strength of the quasimode that is formed. This effect can be seen in the spectrum as an increase in frequency with radius, with transitions occurring from one frequency to the next. Not shown is a higher frequency mode which appears at $r / a \gtrsim 0.5$ for the higher mode numbers $(m=12-15)$. Thus the mode structure cannot be completely described by either the strong coupling (ballooning mode) or weak coupling (Fourier mode) limits, but has some aspects of each. Qualitatively, the variation of $\omega^{*}$ with radius reduces the number of poloidal modes involved in a single quasimode, and limits the radial extent somewhat. Nevertheless, we find that the ensuing quasimode extends over a large radial scale, t.ypically $1 / 3$ to $1 / 2$ of the minor radius.

The radial structure obtained from the interferogram diagnostic ${ }^{33}$. shows peaking of the potential near the rational surface for the observed frequencies. The waveforms are typically highly oscillatory in $r$ and overlap an appreciable number of adjacent rational surfaces. This behavior is a consequence of the weakly damped or unstable, radially extended character of the mode, in contrast to the rapid radial decay of the slab geometry drift mode eigenfunction. Radial interferograms for the $m=12$ mode are shown in Figure 7 (a) and (b) for the toroidal and cylindrical runs. The width of the interfered potential in the toroidal case is approximately twice that in the cylindrical run, demonstrating the radially-extended nature of the toroidal mode. In the toroidal system, the finite radial width of the eigenfunction is caused by the variation of equilibrium quantities with $r$ (such as the diamagnetic frequency), and from truncation of the poloidal mode space.

The interferogram in the poloidal angle shows another aspect of the toroidal nature 
of these modes. Theoretically, strongly coupled modes exhibit "ballooning" towards the ontside of the torus. This ballooning behavior is observed in the simulation, for several frequency ranges and at many radial grid points; a representative interferogram is displayed in Figure 7 (c). For the case shown, the maximum amplitude occurs away from the $\theta=0$ axis (outward direction), although more usually the maximum occurs on the axis. 


\section{IV. $\eta_{i}$ and Trapped Particle Modes}

Recent tokamak experiments utilize Ohmic heating from the toroidal current as well as auxiliary heating such as neutral beam injection (NBI) or ion cyclotron resonance heating (ICRH) in order to obtain high central temperatures. In such tokamak experiments the interior plasma ion temperature is raised quite high-sometimes as much as $20 \mathrm{keV}$. In these plasmas experimentalists t.ypically observe both the anomalous electron heat transport typical of Ohmically heated plasmas (such as the Alcator experiment) as well as anumalous ion heat transport. ${ }^{2}$ Although the existence of temperature gradient driven drift wave instabilities has been known for quite a while, ${ }^{34}$ the experimental observations of anomalous ion heat transport prompted renewed theoretical and computational study "f this class of instability. The drive for the temperature gradient driven modes is usually expressed in terms of $\eta_{j}=\partial \ln T_{j} \partial \ln n_{j}(j=e$ or $i)$, the steepness of the temperature gradient with respect to the density gradient. In this sense the previous section deals with the situation with $\eta_{e}=\eta_{i}=0$. On the other hand the case with $\eta_{j}=\infty$ is referred to as the flat density limit of the temperature gradient instability (TGI). In tokamak plasmas almost always $\eta \geq 1$, although in other devices $\eta<0$ is possible. ${ }^{34,38}$

In this section we compare the plasma behavior with finite $\eta_{j}$ to that with $\eta_{j}=0$. Note that an initial state with a constant $\eta_{j}$ has a temperature profile given by $T_{j}(r)=$ $T_{j 0}\left(n(r) / n_{0}\right)^{\eta}$. We examine runs in both toroidal and cylindrical geometries for finite $\eta_{j}$ in this section. In addition the simulation for the $\eta_{i}$ instability and associated nonlinear: behavior may be carried out either with drift kinetic electron dynamics (as done in the previous section) or with a fluid electron description based on the Boltzmann (adiabatic) electron response.

The Boltzmann electron response is used for the majority of theoretical studies of the $\eta_{i}$ mode, and we refer to these treatments as "classical" $\eta_{i}$ mode theory. Linear kinetic theory shows that the classical $\eta_{i}$ mode theory is a good approximation provided that $\eta_{i}=\eta_{c r i t}$. where $\eta_{c r i t}$ is of order unity. A detailed example of the change from the 
trapped electron mode to the classical $\eta_{i}$ mode behavior as $\eta_{i}$ is increased is given in Figure 1 by Rewoldt and Tang. ${ }^{36}$ Thus it is instructive to compare results for runs with kinetic electrons to those with with adiabatic electrons for low values of $\eta_{i}$. Except for the variation of temperature profile and electron dynamics type, the method of analysis and runs are the same as in the previous section.

\section{A. Simulations with drift kinetic electrons}

See Figure 5 (a) for the radial variation of the safety factor $q$, shear parameter $\dot{s}$, and the t,nroidicity parameter $c$, and Figure 5 (b) for the ion Larmor radius $\rho_{i}$, ratio of ion Larmor radius to density scale length $\rho_{i} / L_{n}$, and ratio of shear length to density scale length $L_{0} / L_{n}$

Here we consider runs using drift kinetic electrons. Two runs are presented: a toroidal run with $\eta_{e}=\eta_{i}=1$, and one with the same pacameters in the cylindrical limit as a control. Although most theory employs adiabatic electron approximations to treat the $\eta_{i}$ instability, it is important to carefully delineate the physics of the $\eta_{i}$ mode when $\eta_{i}$ is near the critical threshold $\left(\eta_{i} \simeq \mathcal{O}(1)\right)$. It is suggested ${ }^{36,37}$ that near the threshold the growth rate of the $\eta_{i}$ mode with kinetic electron dynamics is substantially greater than that with adiabatic electron dynamics.

Simulation parameters are chosen identical to those of the previous $\eta=0$ run (cf. Table 2) with a few exceptions. Due to the temperature gradient, the ion Larmor radius is now a function of radial position, but the gyroradius is chosen in a way such that the average value is the same for each run. Also, the outside mode $(m=15)$ from the previous run was neglected in order to further ensure that boundary effects were minimal.

In Figures 8 (a) and 9 (a) we give contour plots of the measured spectral density as a function of $\omega$ and $r$. A strosig, low frequency mode in the electron diamagnetic direction $\left.\left(\omega^{\prime}, \omega^{\bullet} \sim 1\right) .4\right)$ is observed, spanning an appreciable radial extent $(0.2 \lesssim r / a \lesssim 0.6)$. Here the toroidal coupling induces the formation of a single frequency mode, with a 
spectral density 3-5 times stronger than that observed in the cylindrical case. In this case $\left(\eta_{e}=\eta_{i}=1\right)$, the temperature gradient causes a strong variation of the diamagnetic frequency with radius for a given $m$, with

$$
\omega^{*} / \Omega_{i}=0.0031 m \exp \left[-0.5(3.168 r / a)^{2}\right]
$$

which causes the individual $m$ modes to be more spatially localized than in the $\eta=0$ case. However, the ensemble value of $\omega^{*}$ (obtained by replacing $m$ by $n q(r)$ in the above expression) is roughly constant with radius, promoting strong coupling between modes at different rational surfaces and consistent with the observed mode strength. Some spectral density features are observed at negative frequency, i.e. with the same sign as the inn diamagnetic frequency, but in this case $(\eta=1)$ the negative frequency components are much weaker than those at positive frequency. Clearly almost all of the power is concentrated in the electron diamagnetic branch.

In Figure 8 (b) the spatial average of the spectral density is plotted versus $\omega / \Omega_{i}$ for $\omega>11$. Here the fall off with frequency is not a power-law, due to enhancement of the low frequency response. The lower hybrid mode is seen at $\omega / \Omega_{i}=1.4$ and is much weaker than the low frequency response. Figure 9 (b) shows spatial average of the spectral density plotted versus $\omega / \Omega_{i}$.

In Figures 10 and 11 we show the density and temperature profiles at two times during the run, for the electrons and ions respectively. After an initial relaxation due to $t=0$ non-equilibrium effects, the profiles change slowly in time. In Figure 12 we show the ion particle diffusion, ion and electron heat transport for the $\eta_{i}=1$ run plotted versus radius. The ion thermal diffusivity $\chi_{i}$ is greater than the electron thermal diffusivity $\chi_{e}$ by a factor of about 2 , which in turn is greater than the ion diffusivity $\left(D_{i}\right)$ by a factor if alsout 4 


\section{B. Simulations with adiabatic electrons}

For the remainder of this section, an adiabatic electron response is employed in our simulation runs. This configuration is much closer to that of the conventional theory, and is therefore easier to interpret. The $\eta_{i}=1$ case is particularly interesting in comparison to the previous kinetic electron results, so we shall focus on it separately. In each of these cases, the run parameters are chosen identical to those of previous $\eta=1$ run (cf. Table 2), except for the absence of kinetic electrons and the variation of $\eta_{i}$. Note that the $\eta_{i}$ variation, in addition to affecting the strength of the instability, affects the radial diamagnetic frequency variation through the decrease of $\rho_{i}$ with $r$.

At the beginning of each simulation run in toroidal geometry there is a transient relaxation in the temperature and density profiles, due to non-equilibrium effects, specifically in diamagnetic effects. This relaxation arises from the fact that a Maxwellian with density and temperature profiles as a function of $r$ (as the particles are loaded) is not an equilibrium solution to the zero order Vlasov equation in toroidal geometry when the ion Larmor radius is significant compared with local scale lengths $L_{n}, L_{t}$ and $r$. A perturbation expansion in orders of Larmor radius gives difference terms on the order of $\rho_{i} / r$, $\rho_{i} / L_{n}$, and $\rho_{i} / L_{t}$, consistent with our observations (the exact dependence is rather complicated and will not be presented here). Since our simulation runs do not allow development of an ambipolar electric field, such quick relaxation has little physical effect except for the modification of the background profiles. As a result, the stated values of $\rho_{i}, \eta_{i}$, and . $\omega^{\bullet}$ must be taken with some caution, as they are initial values only. As an example, for the $\eta_{i}=4$ run the initial transient causes relaxation to a value of approximately $\eta_{i}=3$ wer most of the profile. As the run progresses, the value of $\eta_{i}$ further decreases across the profile due to effects of the instability and the associated transport. For simplicity of nomenclature, we shall thus refer to each run in terms of the initial value of $\eta_{i}$ only. Also II)te that transport measurements are taken only after the initial relaxation in order to get a result for fluctuation-induced transport alone (if included, the transport due to the 
initial relaxation can severely bias the result).

Further, we note that due to the strong toroidicity $\left(a / R_{0}=0.4\right)$, hoth ion temperature gradient modes and trapped ion modes are expected to be present in general. The ion bounce frequency is comparable to the observed mode frequency in some cases, leading us to believe the trapped ion effects may be important part of the observed instability. However, separating the destabilizing effects of the trapped ions from that of the in temperature gradient instability in the simulation analysis is practically impossible. Thus, for simplicity we shall simply refer to the " $\eta_{i}$ mode" when discussing the observed phenomena, although the results may apply to the trapped ion mode as well.

\section{1. $\eta_{i}=1$}

The $\eta_{i}=1$ run with adiabatic electrons showed essentially no growth of the electric potential. This result occurs due to the absence of the kinetic electron drive and because $\eta_{i}=1$ is so close to marginal stability for the classical $\eta_{i}$ mode. Further, the spectral density intensity and transport were small compared to the other runs discussed in this work. It is nevertheless interesting to compare this run to the kinetic electron $\eta_{i}=1$ run of the previous section since they differ only by the addition of the electron drive.

Figure 13 (a) shows a contour plot of the spectral density versus $(r, w)$. The spectral intensity of the potential at positive frequency is seen to be 50 times smaller than for the kinetic electron case discussed in the previous section. The plasma response shows some similarity to that of the kinetic electron case, having roughly the same radial extent $(0.05 \lesssim r / a \lesssim 0.55)$, and approximately the same frequency $\left(\omega / \omega^{*} \sim 0.4\right)$. A relatively strong ion feature appears for $r / a \sim 1) .15$; this feature also appeared in the kinetic electron run but was hardly noticeable due to the much stronger response at positive frequency. The power is more or less evenly divided between electron and ion branches, and the individual modes are still recognizable.

Figure 13 (b) shows the spatial average of the spectral density plotted versus $\omega / \Omega_{i}$, 
logarithmic axes, positive $\omega$. The spectral density is observed to decay as $\omega^{-1.5}$. The ion cyclotron frequency is seen at $\omega / \Omega_{i}=1.0$, showing that the high frequency component is substantially weaker than the low frequency response even for this relatively quiet case. Here the lower hybrid mode is missing since the kinetic electron response is absent. The sharp peaks at higher frequencies are artifacts of the diagnostic.

Figure 14 shows the ion density and temperature profiles at two times during the run, well after the initial transient. The profile modification between these two times is clearly small.

2. $\eta_{i}=2,3$

For the $\eta_{i}=2,3$ runs, robust instability was observed, with a maximum growth rate of $\gamma=7.8 \times 10^{-4} \Omega_{i}$ at $r / a=0.45$ for the $\eta_{i}=2$ run and $\gamma=1.4 \times 10^{-3} \Omega_{i}$ at $r / a=0.42$ for $\eta_{i}=3$. The transport coefficients $D$ and $\chi$ were calculated for each and are presented in the following section. The spectral densities are given in Figures $15\left(\eta_{i}=2\right)$ and 16 $\left(\eta_{i}=3\right)$. In these figures, part (a) shows a contour plot of the spectral density $S(r, w)$, with the solid lines representing the effective $\omega^{*}$, and part (b) shows a logarithmic plot of the spatially averaged spectral density as function of $\omega / \Omega_{i}$.

In both cases, a strong mode was observed, with real frequency near $10 / \Omega_{i} \sim 0.003$. The peak energy exceeds that in the $\eta_{i}=1$ run by approximately one and two orders of magnilude for the $\eta_{i}=2$ and $\eta_{i}=3$ runs, respectively. In each case, the observed . mode has a frequency remarkably constant in $r$ compared to the effective diamagnetic frequency, and extends across many rational surfaces. The spectral density is observed to decay as $S(\omega) \sim \omega^{-1.4}$ in each case, for $\omega^{*}<|\omega|<\Omega_{i}$.

3. $\eta_{i}=4$

In this section, we show results from both toroidal and cylindrical $\eta_{i}=4$ runs. The dramatic differences that appear underscore the importance of toroidal effects to $\eta_{i}$ tur- 
bulence and transport.

The $\eta_{i}=4$ (toroidal) run showed the most robust growth of all the runs reported in this work, as well as the highest transport, with a maximum growth rate of $\gamma=2.2 \times 10^{-3} \Omega_{i}$ at $r / a=0.39$. The mode saturates at around $t=2000 \Omega_{i}^{-1}$.

In Figures 17 and 18 are pluts of the spectral density for the toroidal and cylindrical $\eta_{i}=4$ runs, respectively. Part (a) shows a contour plot of the spectral density versus $(r, \omega)$, with the solid lines representing the effective $\omega^{*}$, and part (b) shows a logarithmic

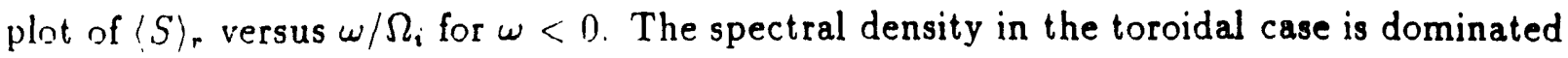
by a nearly constant frequency mode, spanning $0.2<r / a<0.5$, with $\omega / \omega^{*} \sim 0.60$ at the center of the mode. By contrast, the spectral density for the cylindrical case is an order of magnitude weaker and consists of several "blobs" spanning a radial distance of $\delta r / a \sim 0.05$, each with a slightly different frequency. The frequency dependence for the cylindrical case also shows a weak downward trend with increasing radius. Note that the frequency for the cylindrical case is not expected to track $\omega^{*}$ precisely because of finite Larmor radius effects, rather, $\omega^{*}$ represents an upper bound. There is some weak activity for $r / a \sim 0.5$ with $\omega>\omega^{*}$, but this is most likely caused by nonlinear (rather than linear) coupling.

In Figure 19 we plot the growth rate and real frequency as a function of increasing $\eta_{i}$, for the runs given in this section. A nearly linear dependence of $\gamma$ on $\eta_{i}$ is observed, consistent with the results of linear theory. The real frequency $\omega$ shows a nearly flat dependence on $\eta_{i}$, with the exception of the $\eta_{i}=1$ case for which there is no strongly excited toroidal mode. In each simulation, the potential was observed to grow for many poloidal mode numbers $m$ and many radial locations, typically near the rational surface fror each mode. A representative plot of electrostatic field energy as a function of time for the $\eta_{i}=2,3$, and 4 is shown in Figure $20(\mathrm{a}),(\mathrm{b})$, and (c), respectively. In each case there is a clear rise above the noise level as the instability sets in.

As the instability develops, we observe the formation of very large scale vortices 
("streamers") in the electric potential, especially for the $\eta_{i}=4$ run. These structures develop most strongly towards the outside of the torus and are nearly radially aligned there, however, the contours near $\theta= \pm \pi / 2$ show some outward shear caused by the variation of $q(r)$ over the length of the streamer. In Figure 21 we show the variation of the potential in $(r, \theta)$ for four separate points in time, using constant values for each contour. In (a), near the beginning of the linear growth phase, the mode has begun to show a global organization. In (b) the mode has reached maximum amplitude; here the elongated structure is clearly seen. In (c), the mode has saturated and the vortices are in the process of being torn apart. Finally, (d) shows the potential at the end of the run, well past saturation, and the large scale vortices have virtually disappeared.

In Figures 22 and 23 we show the ion density and temperature profiles at two times during the run, for the toroidal and cylindrical runs, respectively. The toroidal run shows noticeably greater levels of transport than in the cylindrical run. Also the profile modificatinn seen in the $\eta_{i}=4$ torsidal run is much greater than for the runs with smaller $\eta_{i}$ shown previously.

In the next set of figures we present the thermal diffusivity $\chi_{i}$ as a function of radius for our simulation runs, as well as the thermal diffusivity observed in experiment. For these plnts $\chi_{i}$ is expressed in units of $\rho_{i}^{2} v_{i} / L_{n}$, the so-called gyro-Bohm scaling. The experimental values are obtained for JT-60, ${ }^{38}$ TFTR, ${ }^{16}$ DIII-D ${ }^{39}$ and JET ${ }^{40}$ (note: unpublished data was used in order to get $\chi_{i}$ versus radius for JT-60). In reducing $\chi_{i}$ to dimensionless form, estimation of the radial averages of $\rho_{i}, v_{i}$, and $L_{n}$ was required. Therefore the experimental values as shown should be taken with some caution.

In Figure 24 are plots of $\chi_{i}(r)$ for the toroidal and cylindrical $\eta_{i}=4$ cases mentioned above. The cylindrical run shows the expected decrease with $r$, while the toroidal run shows an overall upward trend. The large jump seen just to the left of $r / a=0.5$ for the toroidal case coincides with the right edge of the radially extended peak in $S(r, w)$ (note a separate run using 8192 sample particles but otherwise identical parameters found a 
much smaller feature in the toroidal $\chi_{i}$ value here). The thermal diffusivity for both runs shows a large increase for $r / a \succsim 0.5$ (not shown), which we believe may represent edge effects and ior poor statistics in the tail region. A simulation run using $200 K$ particles and otherwise identical parameters resulted in a $\chi_{i}$ with essentially the same features.

In Figure 25 we plot $\chi_{i}(r)$ for toroidal simulation runs of $\eta_{i}=1,2,3$, and 4 . The thermal diffusivity shows a clear upward progression with increasing $\eta_{i}$, with an order of magnitude separation between the $\eta_{i}=4$ and $\eta_{i}=1$ diffusivities at $r / a \sim 0.5$. Finally, in Figures 26 and 27 we compare $\chi_{i}(r)$ for the $\eta_{i}=3$ and $\eta_{i}=4$ cases to that seen in several present-day tokamaks (Figure 27 contains the improved measurement using 8192 sample particles). A strikingly similar radial dependence between experiment and simulation is seen.

\section{Discussion}

The heat transport coefficient $\chi_{i}$ in toroidal geometry is observed to be nearly an order of magnitude greater than that for cylindrical geometry over a wide range of $\eta_{i}$ values, including $\eta_{i}=0$. The cylindrical geometry result for $\chi_{i}$ is about an order of magnitude too low to explain observations from tokamak data, an effect also investigated by Kotschenreuther et al. ${ }^{41}$ In that work, annular toroidal kinetic simulation was used to ubtain a value for $\chi_{i}$ of the same order of magnitude as that seen in tokamak experiments, although the radial variation did not show the characteristic increase in $\chi_{i}$ with $r$ seen in experiment.

The transport (as measured by the $\chi_{i}$ diagnostic) also increased as a function of $\eta_{i}$. The measured $\chi_{i}$ for all runs was seen to increase as the instability developed, then drop after saturation to some finite level. In order to get a steady state result, measurements if $\gamma_{1}$ were made after saturation where possible. Since saturation was just reached by the end of the run for $\eta_{i}=3$, and not reached at all for $\eta_{i}=2$, the $\chi_{i}$ curves for these runs are almost certainly influenced by the effect of the instability (longer runs may be helpful 
liere). For the $\eta_{i}=4$ case, the $\chi_{i}$ measurement thus represents essentially the saturated state value, although some effect of the linear growth phase may still be present. The increase in $\chi_{i}$ with $\eta_{i}$ is most marked towards the outside of the torus $(r / a \gtrsim 0.4)$ for these parameters. The data at larger $r(r / a \gtrsim 0.55)$ is typically omitted because of poor statistics caused by relatively few marker particles there. The $\chi_{i}$ value at small $r$ $(r / a \lesssim 0.1)$ is somewhat suspect for the same reason (a fixed density near $r=0$ implies that the number of particles vanishes there).

The temperature and density profiles are observed to relax much more rapidly as $\eta_{i}$ increases. This is consistent with the observed greater growth and greater measured transport. Likewise the relaxation of the temperature profile for the $\eta_{i}=4$ toroidal case is. much more pronounced than that for the $\eta_{i}=4$ cylindrical case. Note that the observed temperature profile relaxation in the toroidal run is global rather than local, indicating a global process of heat transport is responsible. Simulations to longer times show that as the temperature profile relaxes, $\eta_{i}$ is remains nearly constant with radius.

The toroidal runs show a real frequency $\omega$ that is nearly independent of radius, with an eigenmode structure that is broad compared to the distance between rational surfaces. By contrast, runs in cylindrical geometry were radially localized with significant variation of the eigenfrequency across $r$, as well as a lower value for the spectral density of electrostatic fluctuations. Clearly, the toroidal effects (most notably the coupling of modes at different rational surfaces) have led to the formation of a global "quasimode", evidenced by the nearly constant (in $r$ ) eigenfrequency and growth rate. The smooth variation of the mode intensity contours also supports the view that a global quasimode has formed, consistent with the results of section III.

The observation of a global quasimode in toroidal simulations is consistent with theoretical expectations. ${ }^{7-9,28}$ A more precise comparison is difficult due to the approximations typically made in theory (cf. Section III). Namely, reduction of the equation for electric potential to an ordinary differential equation in the extended poloidal variable $\dot{\theta}$ elim. 
inates all information about radial variation (translational invariance). This treatment results in a quasimode that extends radially over many rational surfaces but is otherwise of undetermined radial width. Only by taking higher order effects (typically not done) into account can a finite radial width be attained. Such effects, which serve to decorrelate the mode radially, include the variation of equilibrium quantities such as $\omega^{*}$ with radius or nunlinearities. A radial mode width on the order of $\left(d \ln \omega^{*} / d r\right)^{-1}$ or $\left(d^{2} \ln \omega^{*} / d r^{2}\right)^{-1 / 2}$ is thereby expected. ${ }^{28}$ implying that as $\eta_{i}$ is increased the global mode width decreases (everything else being equal). This argument is in conflict with the simulation results presented here. As $\eta_{i}$ is increased the mode width is seen to in fact increase (cf. Figure 17). Of course the mode strength increases dramatically with increasing $\eta_{i}$, which will also have an effect on mode width.

In the $\eta_{i}=3$ and $\eta_{i}=4$ runs the radial extent of the mode is sufficiently large so that the absolute value of the frequency exceeds that of the local (initial) diamagnetic drift frequency $\left|\omega_{i}^{*}\right|$ for $r / a \gtrsim 0.4$. Although this must be viewed with some caution as the profiles are evolving in time, it represents a possibly important mechanism for energy transport. In the interior region, where $|\omega|<\left|\omega_{i}^{*}\right|$, the global mode grows by extracting kinetic energy from the (hotter) plasina there. In the outer region where $|\omega| \sim\left|\omega_{i}^{*}\right|$ the mode releases the wave energy to the colder plasma there. The net effect is that the global mode can provide a conduit for heat flow (i.e. a heat pipe) from the interior to the exterior of the plasma. This heat pipe effect may be an important factor in the observed values for $\chi_{i}$, and explain the difference between transport in the toroidal and cylindrical systems.

In a cylindrical system there is no global mode structure; each eigenmode is localized so that heat transport is only local. Further, since each eigenmode is independent the eigenmode width scales with the ion Larmor radius. Thus the heat transport decreases with radius (i.e. decreasing temperature), and on a global scale is random rather than coherent. This view is consistent with the simulation results presented here and by other 
authors."

In a toroidal system, however, two effects combine to dramatically elevate the heat transport while giving it a global character. These effects are (a) the global nature of the mude caused by poloidal mode coupling, and (b) extension of the global mode into Incally stable regions. Recall that diffusivity is measured in units of $[d]^{2} /[t]$, where $[d]$ is distance and $|t|$ is time. The enhanced global nature of the mode increases the distance lieat can flow, while the extension of the mode into regions where it is heavily Landau damped increases the rate $(1 /[t])$ at which the wave energy may be transferred to the plasma. Both of these effects may contribute to the elevation of $\chi_{i}$. In our simulations the radial mode width is observed to decrease with decreasing $\eta_{i}$, and the observed heat transport is correspondingly reduced, consistent with this explanation. A related effect seen in the $\eta_{i}=4$ run is a jump in $\chi_{i}$ to the outside of the rational surfaces in the system. This can be interpreted as a local heat sink in light of the comments made above.

Finally, we also observe the mode strength to increase substantially near the threshold $\eta_{i}$ in the presence of kinetic electrons. Consequent transport is also substantially higher. In the kinetic electron run we can compare the ion thermal diffusivity $\chi_{i}$, electron thermal diffusivity $\chi_{e}$, and ion particle diffusivity $D_{i}$, as the free movement of electrons allows for both electron heat transport as well as ambipolar motion of both species. As mentioned previously, we found in this case that $\chi_{i}>\chi_{e}>D_{i}$, where $D_{i} \simeq D_{e}$. In our normalization $D_{i}$ can be considered the same as the convective ion heat transport coefficient, while $\chi_{i}$ or $\chi_{e}$ represent the sum of the convective and conductive ion or electron heat transport coefficient, respectively. However, in most of the simulation cases the convective contribution to the heat, transport is much smaller than the sum; thus in these cases $\chi_{i}$ or $\chi_{e}$ are effectively the measure of the conductive heat transport. Both the convective and crinductive transport are enhanced due to the fluctuations. 


\section{Summary}

In conclusion, particle simulation of toroidal plasmas has been used to observe drift/trappedparticle driven turbulence and transport as $\eta_{i}$ is increased from zero to large positive values. For toroidal runs the value $1 \leq \eta_{i} \leq 2$ marks the transition from a predominantly electron drift wave fluctuation spectrum to an ion drift wave fluctuation spectrum. The transition also marks the change from little to large ion heat transport. These results are consistent with the standard theory. ${ }^{36.42}$ Our simulation runs involving kinetic electrons show comparatively higher level of fluctuations and thus also higher level of transport. This effect is worth mentioning, as most theoretical and computational work to date employ the adiabatic electron response or the non-dynamical electron $\left(1-i \delta_{k}\right)$ response for conceptual and analytical simplicity

Our simulation run with kinetic electrons for $\eta_{e}=\eta_{i}=0$ shows clear evidence of the theoretically predicted toroidicity-induced mode. We see activity at several frequencies below the diamagnetic frequency, separated by unoccupied "bands" in frequency space. The lowest frequency mode occurs at a real and imaginary frequency close to that predicted by linear theory. Further, these modes show the radial elongation and outward ballooning characteristic of a toroidicity-induced mode.

For $\eta_{i}>1$ the plasma is shown to be strongly unstable to temperature gradient driven modes. Simulation runs in a toroidal system show marked differences from runs in a cylindrical system. The eigenfrequency in the toroidal runs was found to be nearly independent of the radius over distances long compared to the rational surface spacing. For large $\eta_{i}$ runs we observed the growth of radially extended, phase-locked modes (i.e. "streamers"), which broke up in the nonlinear phase. The heat transport peaked during the instability, then dropped to a (near) steady state value. The measured $\chi_{i}$ in the toroidal system was enhanced by nearly an order of magnitude over that in the cylindrical system. Further, the toroidal $\chi_{i}$ is seen to be an increasing function of radius in agreement with experimental observations. 
We argue that the observed heat transport is directly linked to the observed global quasimodes that are formed in the presence of strong poloidal mode coupling. The development of structures that are very long radially implies that energy can travel long distances, giving rise to an increase in $\chi_{i}$. Further, at high values of $\eta_{i}$ the mode can be robustly unstable yet stretch into a region of high dissipation (through ion Landau damping), increasing the rate of energy transfer. These observations may qualitatively explain why the heat conductivity $\chi_{1}$ in a torus tends to be a increasing function of radius as $\eta_{2}$ is raised, as seen both in our simulation runs and in experiment. On the other hand, nontoroidal simulations (slab or cylinder) or even the so-called annular torus (localized portion of a torus) simulations do not show an increasing $\chi_{i}$ as a function of minor radius. In slab or cylindrical simulation, the local value of the ion Larmor radius $\rho_{i}(x)$ determines the localized mode width. Since $\rho_{i}$ is a decreasing function of radius due to the effect of the temperature gradient, the value for $\chi_{i}$ is also observed to decrease with radius.

In a more realistic system, modes are distributed beyond the radial region we considered in the present investigation. In this case we anticipate the rise of $\chi_{i}$ with radius to continue. A physical plasma also possess a large number of toroidal Fourier $(n)$ modes, which will have an effect on the results reported here. When multiple toroidal n-modes exist, particles simultaneously experience more than one of the coupled (in $m$ ) quasiglobal modes seen in our simulations. This should lead to a smoother radial variation of the heat conductivity than in the present case (cf. Figure 27) as well as a higher level of iverall transport. Note, however, that the increase in $\chi_{i}$ caused by the presence of many $n$ modes should be mitigated somewhat (perhaps entirely) by the decorrelation caused by nonlinear coupling of the modes. These expectations are confirmed by simulation runs involving multiple $n$ modes, to be presented in a later work.

The simulations performed thus far with TPC appear to agree with the theoretical predictions in regimes where the linear effects dominate and the radial inhomogeneities are not ton severe. In other regimes where the physics is much less well understood, 
important links between transport and fluctuations are seen. Several improvements to the algorithm are currently underway, which will reduce the cost in running the code as well as inprove accessibility to actual tokamak parameter regimes. We do not, however, expect that the physical characteristics of the fluctuation and transport processes reported here will be significantly changed by these improved algorithms. 


\section{Acknowledgements}

This work was supported in part by the US Department of Energy grant DE-FG05801 ET53088 and in part by National Science Foundation (NSF) grant ATM 88-11128. A portion of this work was also carried out under the sponsorship of the Japan Institute for Fusion Theory (JIFT) exchange program and under the Science and Technology Agency of Japan (STA) fellowship program. 


\section{References}

${ }^{1}$ E. Apgar, B. Coppi, A. Gondhalekar, H. Helava, D. Komm, F. Martin, B. Montgomery, D. Pappas, R. Parker, and D. Overskei, High-density and collisional plasma regimes in the alcator programme, in Plasma Physics and Controlled Nuclear Fusion Research 1976, volume 1, pages 247-257, International Atomic Energy Agency, Vienna, 1977.

2JET TEAM, Recent JET results and future prospects, in Plasma Physics and Controlled Vuclear Fusion Research 1990, volume 1, pages 27-52, International Atomic Energy Agency, Vienna, 1991.

${ }^{3}$ L. I. Rudakov and R. Z. Sagdeev, Soviet Physics - Doklady 6, 415 (1961).

${ }^{4}$ L. D. Pearlstein and H. L. Berk, Physical Review Letters 23, 220 (1969).

${ }^{5}$ D. W. Ross and S. M. Mahajan, Physical Review Letters 40, 324 (1978).

${ }^{6}$ K. T. Tsang, P. J. Catto, J. C. Whitson, and J. Smith, Physical Review Letters 40, 327 (1978).

${ }^{7}$ J. B. Taylor, Does magnetic shear stabilize drift waves?, in Plasma Physics and Controlled Nuclear Fusion Research, pages 323-329, International Atomic Energy Agency, 1977.

${ }^{8}$ D. Choi and W. Horton, Physics of Fluids 23, 356 (1980).

${ }^{9}$ L. Chen and C. Z. Cheng, Physics of Fluids 23, 2242 (1980).

I"R. J. Hastie, K. W. Hesketu, and J. B. Taylor, Nuclear Fusion 18, 1223 (1979).

"C. Z. Cheng and L. Chen, Physics of Fluids 23, 1770 (1980).

${ }^{12}$ C. Z. Cheng and K. T. Tsang, Nuclear Fusion 21, 643 (1981).

${ }^{13} \mathrm{~K}$. W. Hesketh, Nuclear Fusion 21, 275 (1981). 
1'T. J. Schep and .Y. Venema, Plasma Physics and Controlled Fusion 27, 653 (1985).

${ }^{15}$ M. C. Zarnstorff, C. W. Barnes, P. C. Efthimion, G. W. Hammett, W. Horton, R. A. Hulse. D. K. Mansfield, E. S. Marmar, K. M. McGuire, G. Rewoldt, B. C. S. E. J. Synakowski, W. M. Tang, J. L. Terry, X. Q. Xu, M. G. Bell, M. B. N. L. Bretz, R. Budny, C. E. Bush, P. H. Diamond, R. J. Fonck, E. D. Fredrickson, H. P. Furth, R. J. Goldston, B. Grek, R. J. Hawryluk, K. W. Hill, H. Hsuan, D. W. Johnson, D. C. McCune, D. M. Meade, D. Mueller, D. K. Owens, H. K. Park, A. T. Ramsey, M. N. Rusenbluth, J. Schivell, G. L. Schmidt, S. D. Scott, G. Taylor, and R. M. Wieland, Advances in transport understanding using perturbative techniques in TFTR, in Flasma Physics and Controlled Nuclear Fusion Research 1990, volume 1, pages 109122, International Atomic Energy Agency, Vienna, 1991.

${ }^{18}$ S. D. Scutt, V. Arumasalam, C. W. Barnes, M. G. Bell, M. Bitter, R. Boivin, N. L. Bretz, R. Budny, C. E. Bush, A. Cavallo, T. K. Chu, S. A. Cohen, P. Colestock, S. L. Davis, D. L. Dimock, H. F. Dylla, P. C. Efthimion, A. B. Erharardt, R. J. Fonck, E. Fredrickson, H. P. Furth, R. J. Goldston, G. Greene, B. Grek, L. R. Gisham, G. Hammett, R. J. Hawryluk, H. W. Hendel, K. W. Hill, E. Hinnow, D. J. Hoffman, J. Hosea, R. B. Howell, H. Hsuan, R. A. Hulse, K. P. Jaehnig, A. C. Janos, S. J. Kilpatrick, P. H. LaMarche, B. LeBlanc, R. Little, D. M. Manos, D. K. Mansfield, E. Mazzucato, M. P. McCarthy, D. C. McCune, K. McGuire, D. H. McNeill, D. M. Meade, S. S. Medley, D. R. Mikkelsen, R. Motley, D. Mueller, J. A. Murphy, Y. Nagayama, R. Nazakian, D. K. Owens, H. Park, A. T. Ramsey, M. H. Redi, A. L. Roquemore, P. H. Rutherford, G. Schilling, J. Schivell, G. L. Schmidt, J. Stevens, B. C. Stratton, M. Ulrickson, S. von Goeler, R. Wieland, II. Williams, J. R. Wilson, K. L. Wong, S. Yoshikawa, K. M. Young, M. C. Zarnsturf, and S. J. Zweben, Physics of Fluids B 2, 1300 (1990).

${ }^{17}$ WV. Horton, Jr., D. Choi, and W. Tang, Physics of Fluids 24, 1077 (1981). 
${ }^{18}$ M. J. LeBrun, Drift Wave Simulation in Toroidal Geometry, PhD thesis, University of Texas at Austin, 1988.

${ }^{19}$ W. M. Tang, Nuclear Fusion 18, 1089 (1978).

${ }^{20}$ R. D. Sydora, J. N. Leboeuf, and T. Tajima, Physics of Fluids 28, 528 (1985).

${ }^{21}$ D. W. Rnss and W. H. Miner, Physics of Fluids 20, 1957 (1977).

${ }^{22}$ W. Horton, R. Estes, H. Kwak, and D. Choi, Physics of Fluids 21, 1366 (1978).

${ }^{23}$ L. Chen, M. S. Chance, and C. Z. Cheng, Nuclear Fusion 20, 901 (1980).

${ }^{24}$ K. W. Hesketh, Nuclear Fusion 20, 1013 (1980).

${ }^{25}$ J. W. Connor, R. J. Hastie, and J. B. Taylor, Plasma Physics 22, 757 (1980).

${ }^{28}$ T. J. Schep, B. J. Braams, and F. Pegoraro, Physics of Fluids 25, 1871 (1982).

${ }^{2 i}$ T. J. Schep and M. Venema, Plasma Physics and Controlled Fusion 27, 211 (1985).

${ }^{28} \mathrm{~J}$. W. Connor and J. B. Taylor, Physics of Fluids 30, 3180 (1987).

${ }^{29} \mathrm{Y}$. C. Lee and J. WV. V. Dam, Kinetic theory of ballooning instabilities, in Proceedings of the Fintte Beta Theory Workshop, pages 93-101, U.S. Department of Energy, 1979.

${ }^{30} \mathrm{~J}$. W. Connor, R. J. Hastie, and J. B. Taylor, Proceedings of the Royal Society of London 365, 1 (1979).

${ }^{31}$ A. Hirose and S. G. Tsotsonis, Nuclear Fusion 30, 2063 (1990).

32.J. P. Burg, Maximum Entropy Spertral Analysis, PhD thesis, Stanford University, Palo Alto, C.A, 975 .

${ }^{33}$ V. K. Decyk, J. .Y. Dawson, and G. J. Morales, Physics of Fluids 22, 507 (1979). 
${ }^{34}$ A. B. Mikhailovskii, Theory of Plasma Instabilities, volume 2, Consultants Bureau, New York, 1974.

${ }^{35}$ A. Y. Aydemir, H. L. Berk, V. Mirnov, O. P. Pogutse, and M. N. Rosenbluth, Physics of Fluids 30, 3083 (1987).

${ }^{36}$ G. Rewoldt and W. Tang, Physics of Fluids B 2, 318 (1990).

${ }^{37}$ W. IOrtun, J. E. Sedlak, D. Choi, and B. Ilong, Physics of Fluids 28, 3050 (1985).

${ }^{38}$.IT-611 TFAM, Recent experiments in JT-60, in Plasma Physics and Controlled Nuclear Fusion Research 1990, volume 1, pages 53-68, International Atomic Energy Agency, Vienna, 1991.

${ }^{39}$ 'r. K. Kurki-Suonio, R. J. Groebner, and K. H. Burrell, Nuclear Fusion 32, 138 (1992).

${ }^{40}$ B. Balet, D. A. Boyd, D. J. Campbell, C. D. Callis, J. P. Christiansen, J. G. Cordey, W. G. G. Core, A. E. Costley, C. A. Cottrell, A. W. Edwards, T. Elevant, L. G. Eriksson, T. Hellsten, O. N. Jarvis, P. P. Lallia, K. Lawson, C. Lowry, P. D. Morgan, P. Nielsen, G. Sadler, D. F. H. Start, P. R. Thomas, K. Thomsen, M. von Hellermann, and H. Weisen, Nuclear Fusion 30, 2034 (1990).

${ }^{41}$ M. Kotschenreuther, H. L. Berk, R. Denton, S. Hamaguchi, W. Horton, C. Kim, M. LeBrun, P. Lyster, S. Mahajan, W. H. Miner, P. J. Morrison, D. W. Ross, R. D. Sydora, T. Tajima, J. B. Taylor, P. M. Valanju, H. V. Wong, S. Y. Xiao, and Y. Zhang, Novel computational techniques to predict transport in confinement devices and applications to ion temperature gradient driven turbulence, in Plasma Physics and Controlled Nuclear Fusion Research 1991), volume 2, pages 361-366, International Atomic Energy Agency, Vienna, 1991.

${ }^{42}$ B. IIong, D. Choi, and W. Horton, Physics of Fluids 29, 1872 (1986). 


\begin{tabular}{|l|l|l|}
\hline profile & configuration & fluctuation \\
\hline$\eta_{i}=11$ & toroidal & toroidal drift, trapped $e^{-}, i^{+}$ \\
$\eta_{i}=0$ & cylindrical & slab-like drift \\
$\eta_{i}=1$ & toroidal & toroidal drift, trapped $e^{-}, i^{+}, \eta_{i}$ mode \\
$\eta_{i}=1$ & cylindrical & slab-like drift, $\eta_{i}$ mode \\
$\eta_{i}=1,2,3,4$ & toroidal, Boltzmann $e^{-}$response & fluid-like drift, trapped $e^{-}, i^{+}, \eta_{i}$ mode \\
$\eta_{i}=4$ & cylindrical, Boltzmann $e^{-}$response & fluid-like drift, $\eta_{i}$ mode \\
\hline
\end{tabular}

Table 1: Summary of simulation runs 


\begin{tabular}{|c|c|}
\hline reference value & parameter \\
\hline$n= \pm 9$ & toroidal mode number \\
\hline$m=( \pm) 5-14$ & poloidal mode number \\
\hline$a R_{0}=0.4$ & aspect ratio \\
\hline$m / M=1 / 100$ & ratio of electron to ion mass \\
\hline$\left\langle T_{e} / T_{i}\right\rangle=1$ & ratio of electron to ion temperature \\
\hline$\left\langle\omega_{e} / \Omega_{i}\right\rangle=10$ & ratio of plasma to ion cyclotron frequency \\
\hline$\lambda_{e} / \rho_{0}=1$ & ratio of Dehye length to ion Larmor radius \\
\hline$\left\langle\rho_{i} / a\right\rangle \sim 0.008$ & ratio of ion Larmor radius to minor radius \\
\hline$\left\langle k_{\theta} \rho_{\mathbf{o}}\right\rangle \sim 0.2$ & perpendicular wavenumber \\
\hline$\epsilon_{n}\left(\equiv \frac{L_{n}}{R_{0}}\right) \sim 0.1$ & toroidicity parameter \\
\hline$\dot{g}\left(\equiv \frac{r q^{\prime}}{a}\right) \sim 1.0$ & shear parameter \\
\hline$\left\langle\rho_{i} / L_{n}\right\rangle \sim 0.025$ & ratio of ion Larmor radius to density scale length \\
\hline$\pi \sim 3$ & ratio of circumference of a circle to its diameter \\
\hline $\begin{array}{l}\frac{\operatorname{mi}}{\Delta_{r}^{2}} \sim 0.03 \text { (in center) } \\
\partial_{r} \sim 004 \text { (in center) }\end{array}$ & $\begin{array}{l}\text { Distance between rational surfaces } \\
\text { Radial grid spacing. }\end{array}$ \\
\hline
\end{tabular}

Table 2: Parameters for simulation runs 


\section{Figure Captions}

1. Toroidal coordinate system.

2. Schematic of $(r, \theta)$ grid for typical case.

3. Eigenmode structure of the potential: (a) Simulation eigenfunction for the $m=$ 2 even mode. (b) Thenretical eigenfunction from shooting code. The solid lines represent the real parts and the dashed lines represent the imaginary parts.

4. Simulation parameters :(a) Initial electron density profile, (b) nonuniform grid, and (c) number of particles per cell. The grid and density profile are plotied versus radial variable; the particles per cell is plotted versus radial cell number. The mode rational surface locations are indicated by the marks above the nonuniform grid ticks.

5. Radial variation of simulation quantities, from top to bottom: (a) safety factor $q$, shear parameter $(\dot{\jmath})$, toroidicity parameter $\epsilon_{n} ;(b)$ ion Larmor radius density $\rho_{i}$, ratio of ion Larmor radius to density scale length $\rho_{i} / L_{n}$, ratio of shear length to density scale length $L_{s} / L_{n}$ all for $\eta_{i}=1 ;$ (c) same as (b) for $\eta_{i}=4$. Each is plotted versus $r ; a$

6. Frequencies and power spectrum obtained from spectral analyzer: (a) Spatially integrated power as a function of $\omega / \Omega_{i}$. (b) Spectral density maxima versus $\omega / \Omega_{i}$ for the $m=7$ mode. (c) $w i \Omega_{i}$ at maximum power as a function of radius for the $m=7$ through $m=11$ modes. The arrows mark the location of the mode rational surfaces $(q=m / n=7.9$ through $11 / 9)$.

i. Interferograms of the potential for the (a) toroidal run and (b) cylindrical run. These are plotted versus $r$ for the $m=12$ mode, both real part (solid line) and imaginary 
(dotted line). (c) Amplitude interferogram of the potential plotted versus $\theta$ for a given value of $r$.

8. Spectral density for kinetic electron simulation, $\eta_{i}=1$ : (a) Contour plot of the spectral density as a function of $(r, w)$. The solid line shows the effective (ensemble averaged, $m \rightarrow n q(r)) \omega_{\tau_{i}}^{*} / \Omega_{i}$. (b) Spatial average of the spectral density plotted versus $\omega / \Omega_{i}$. logarithmic axes, positive $\omega$.

9. Spectral density for kinetic electron simulation, $\eta_{i}=1$ : (a) Contour plot of the spectral density as a function of $(r, \omega)$. The solid line shows the effective (ensemble averaged, $m \rightarrow n q(r)) \omega_{T_{i}} / \Omega_{1}$. (b) Spatial average of the spectral density plotted versus $\omega / \Omega_{i}$.

11. Profiles for kinetic electron simulation, $\eta_{i}=1$ : (a) Electron density and (b) temperature versus $r / a$.

11. Profiles for kinetic electron simulation, $\eta_{i}=1$ : (a) Ion density and (b) temperature versus $r / a$.

12. Ion particle diffusion $\left(D_{i}\right)$, ion and electron heat transport $\left(\chi_{i}\right.$ and $\left.\chi_{e}\right)$ for $\eta_{i}=1$ run with $400 \mathrm{k}$ ions and $400 \mathrm{k}$ drift kinetic electrons.

13. Spectral density for adiabatic electron simulation, $\eta_{i}=1$ : (a) Contour plot of the spectral density versus $(r, \omega)$. (b) Spatial average of the spectral density plotted versus $\omega / \Omega_{i}$, logarithmic axes, positive $\omega$.

14. Profiles for adiabatic electron simulation, $\eta_{i}=1$ : (a) Ion density and (b) temperature versus $r / a$.

15. Spectral density for adiabatic electron simulation, $\eta_{i}=2$ : (a) Contour plot of the spectral density versus $\left(r, w^{\prime}\right)$. Vntice the global mode at a single frequency. The srilid lines represent $\omega^{\bullet}$ for this run. (b) Spatial average of the spectral density plotted versus $\omega / \Omega_{i}$, logarithmic axes, positive $\omega$. 
113. Spectral density for adiabatic electron simulation, $\eta_{i}=3$ : (a) Contour plot of the spectral density versus $(r, \omega)$. Notice the global mode at a single frequency. The solid lines represent $\omega^{*}$ for this run. (b) Spatial average of the spectral density plotted versus $\omega / \Omega_{i}$, logarithmic axes, positive $\omega$.

17. Spectral density for adiabatic electron simulation, $\eta_{i}=4$, toroidal geometry: (a) Contour plot of the spectral density versus $(r, \omega)$. Notice the global mode at a single frequency. The solid lines represent $\omega^{\bullet}$ for this run. (b) Spatial average of the spectral density plotted versus $\omega / \Omega_{i}$, logarithmic axes, positive $\omega$

18. Spectral density for adiabatic electron sim lation, $\eta_{i}=4$, cylindrical geometry: (a) Contour plot of the spectral density vers,,$(r, \omega)$. Notice the separate peaks present for each poloidal mode $m$. The solid nes represent $\omega^{*}$ for this run. (b) Spatial average of the spectral density plotter versus $\omega / \Omega_{i}$, logarithmic axes, positive $\omega$.

19. Growth rate versus $\eta_{i}$ simulation.

21). Representative plots of electrostatic field energy for growing modes in (a) $\eta_{i}=2$, (b) $\eta_{i}=3$, and (c) $\eta_{i}=4$. Notice exponentiation of field energy followed by saturation.

21. Potential contours for the toroidal run at (a) the beginning of linear growth, (b) the end of linear growth with streamer structures, (c) saturation with the start of streamer breakup, (d) saturation after streamer breakup.

22. Profiles for adiabatic electron simulation, $\eta_{i}=4$, toroidal geometry:(a) Ion density and (b) temperature versus $r / a$.

23. Profiles for adiabatic electron simulation, et $a_{i}=4$, cylindrical geometry:(a) Ion density and (b) temperature versus $r / a$.

21. Heat transport: comparison of $\chi_{1}(r)$ versus $r / a$ for cylindrical and toroidal runs. $8110 \mathrm{k}$ ions, adiabatic electrons. $\eta_{1}=4$ 
25. Heat transport $\chi_{2}(r)$ versus $r / a$ for various values of $\eta_{i}$ in toroidal runs. Notice dramatically reduced transport for $\eta_{i}=1$.

26. Comparison of TPC $\eta_{i}=3$ simulation to JT-60, ${ }^{38} \mathrm{TFTR}^{19}$ DIII-D ${ }^{38}$ and JET ${ }^{40}$ experimental heat transport. Simulation used $800 \mathrm{k}$ ions, and adiabatic electrons.

27. Comparison of TPC $\eta_{i}=4$ simulation to JT-60, ${ }^{38}$ TFTR, ${ }^{16}$ DIII-D ${ }^{39}$ and JET ${ }^{40}$ experimental heat transport. Simulation used $800 \mathrm{k}$ ions, and adiabatic electrons. 


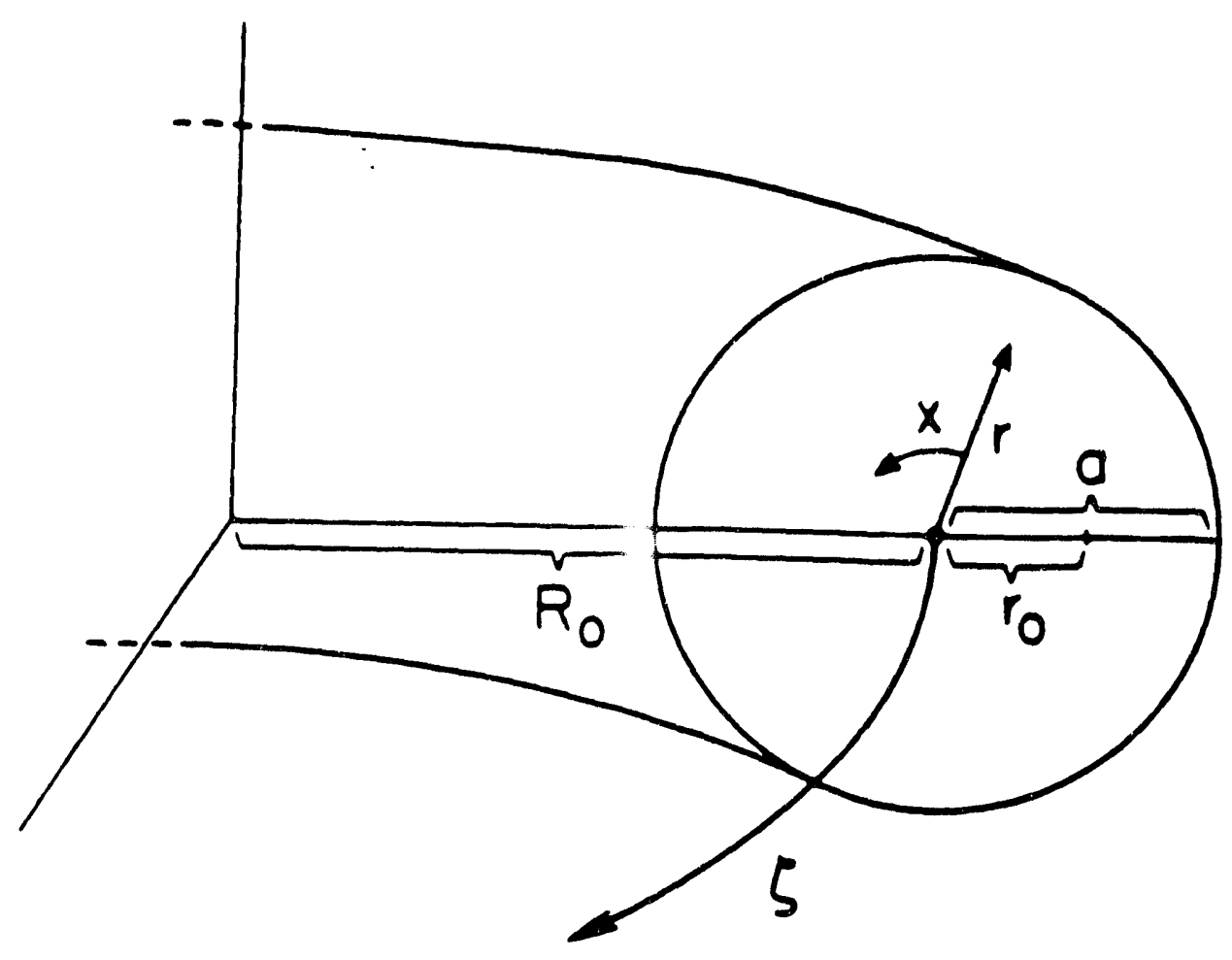

Fig. 1 


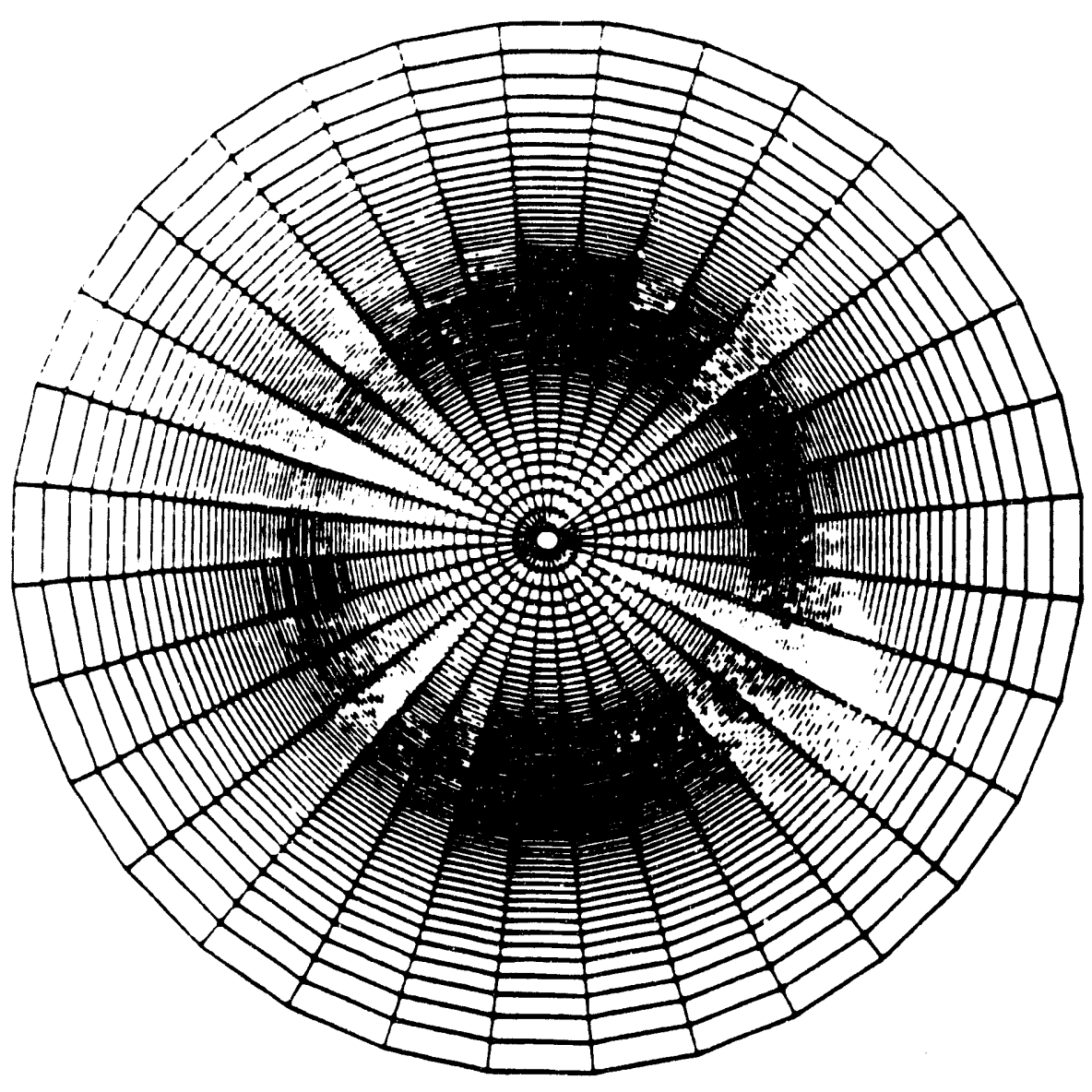

Fig. 2 

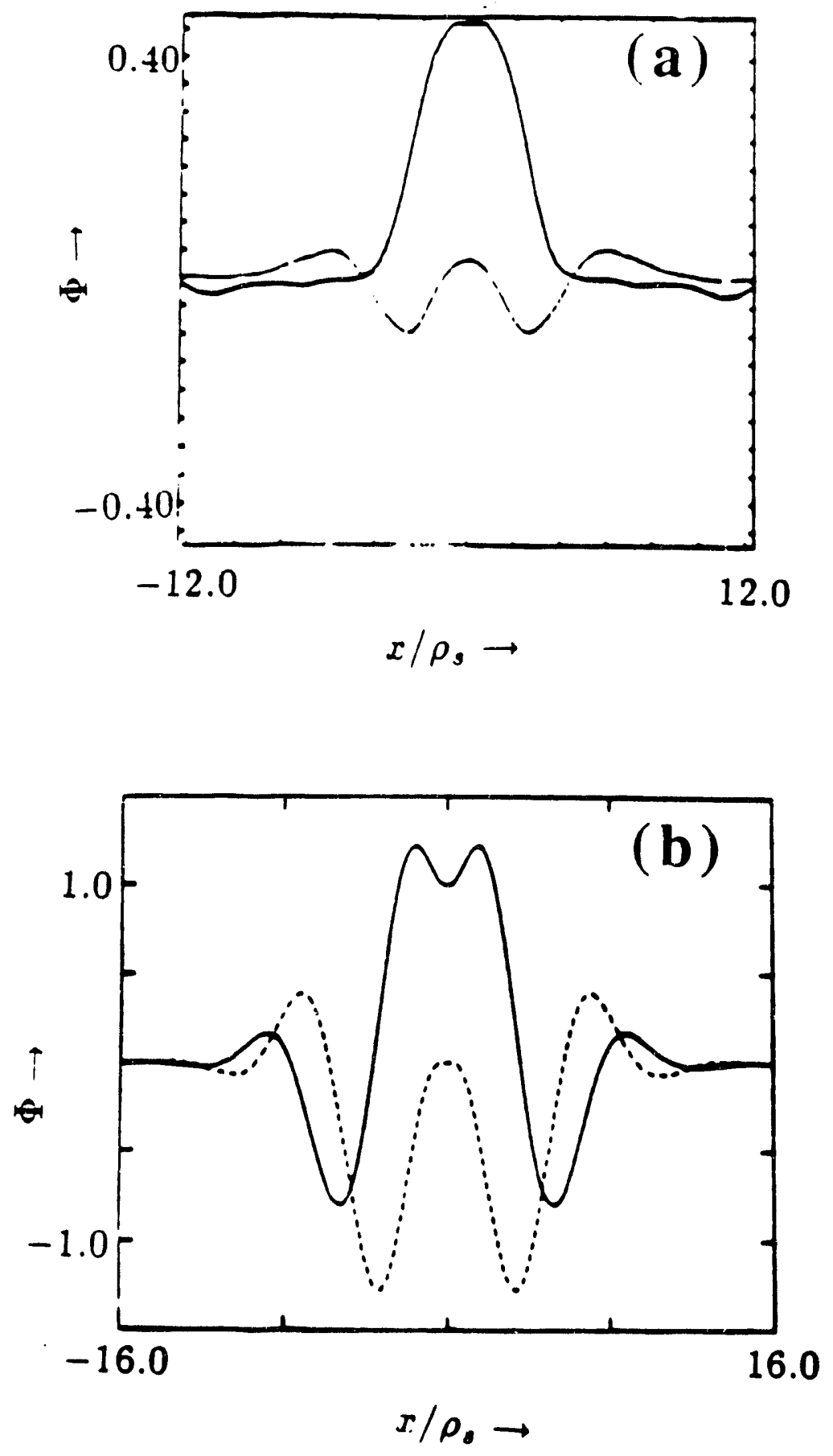

Fig. 3 

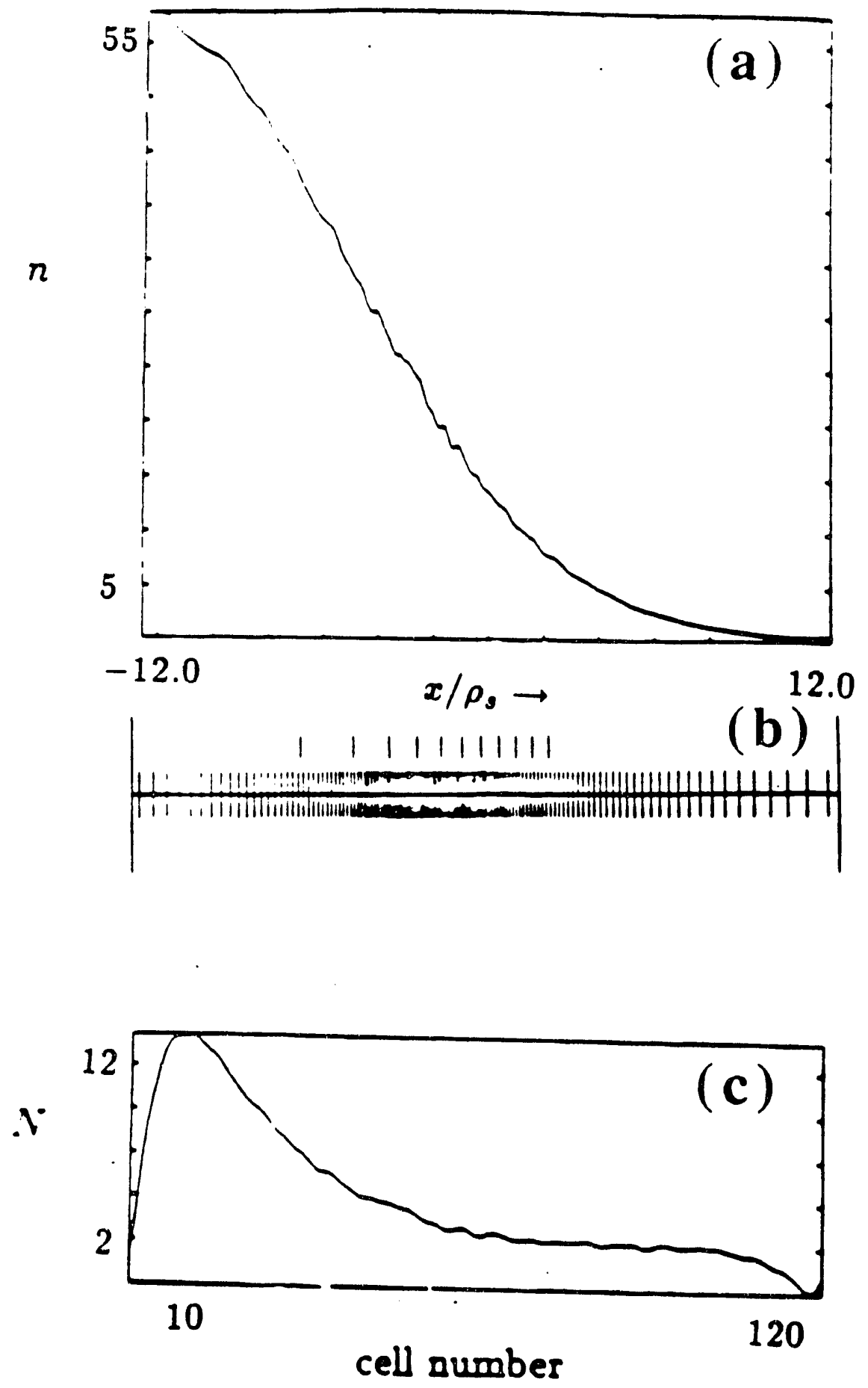

Fig. 4 

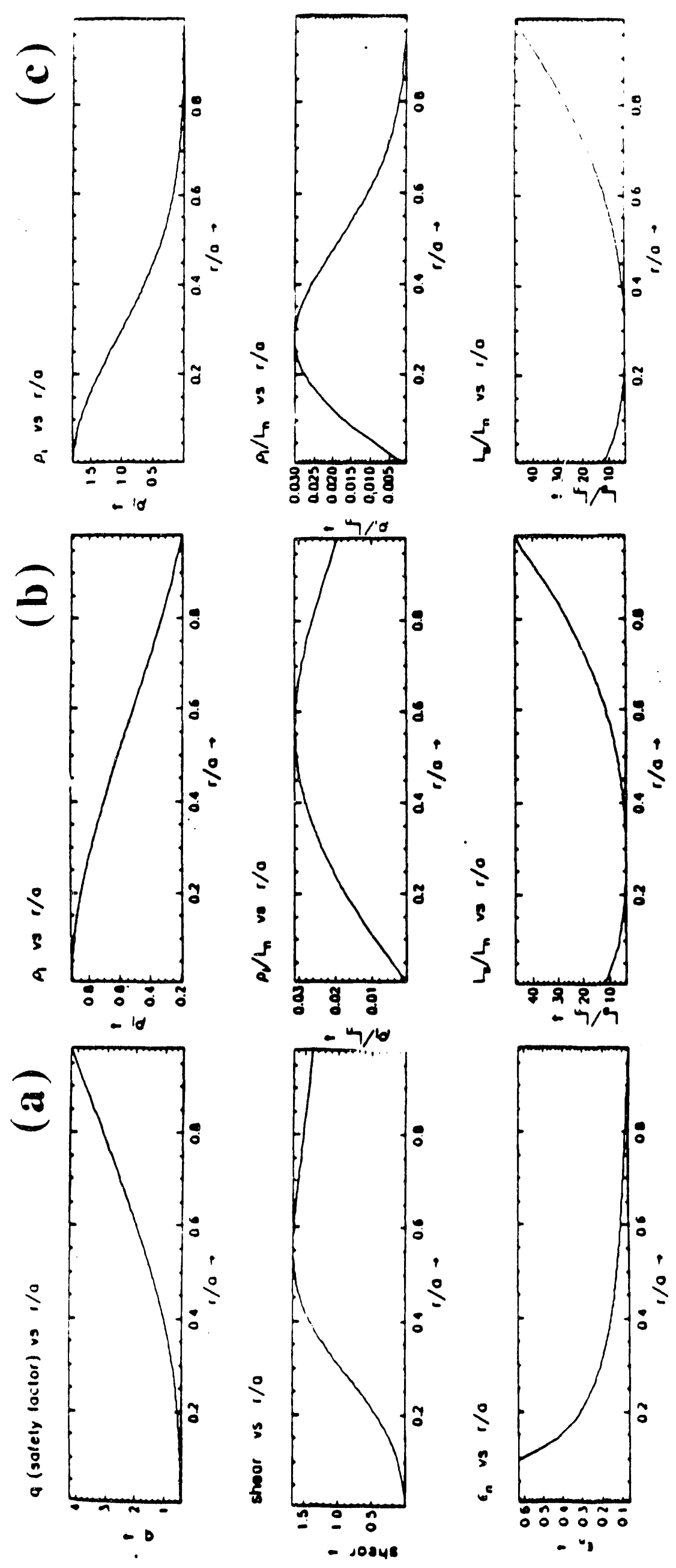

Fig. 5 


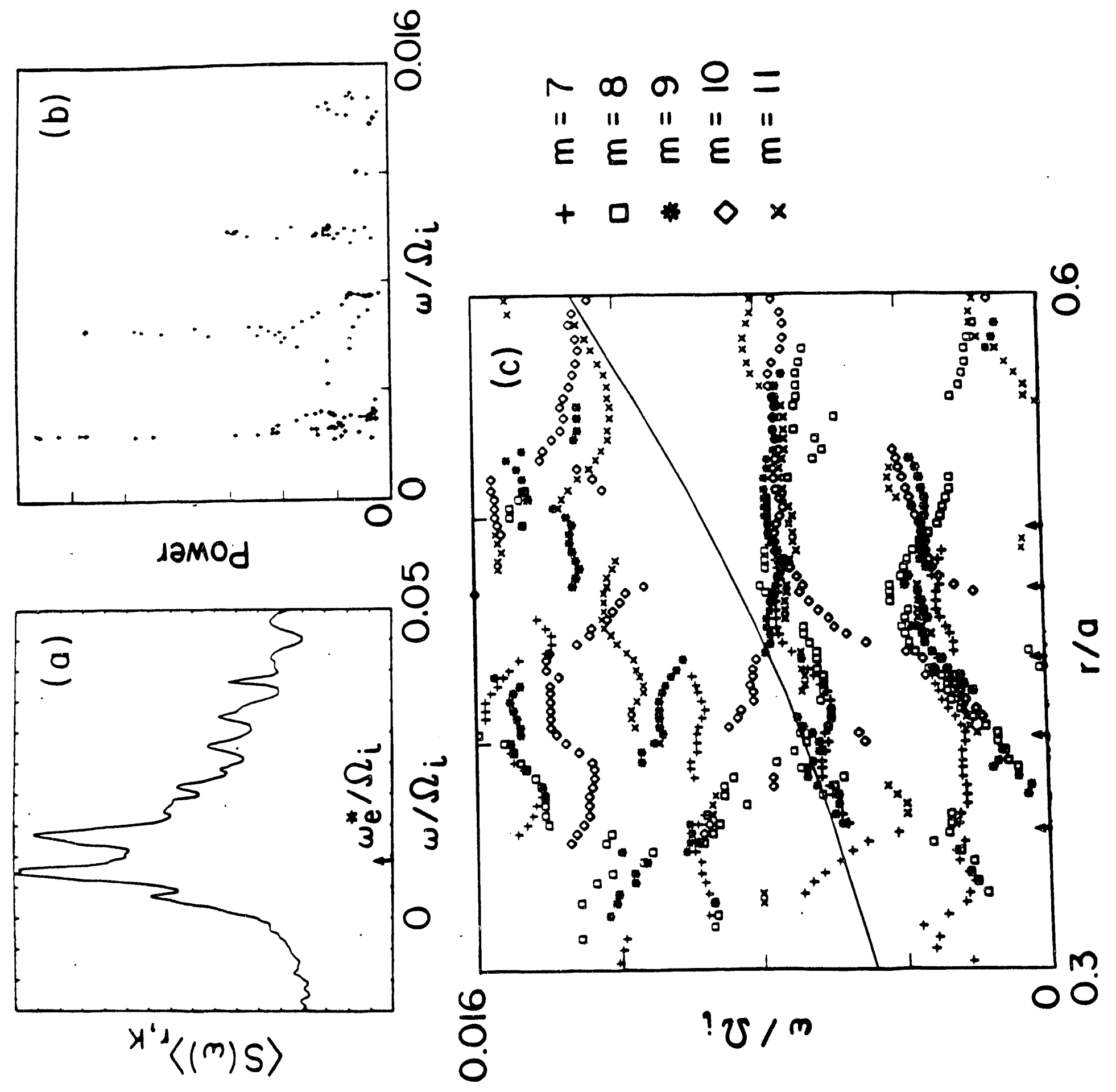

Fig. 6 


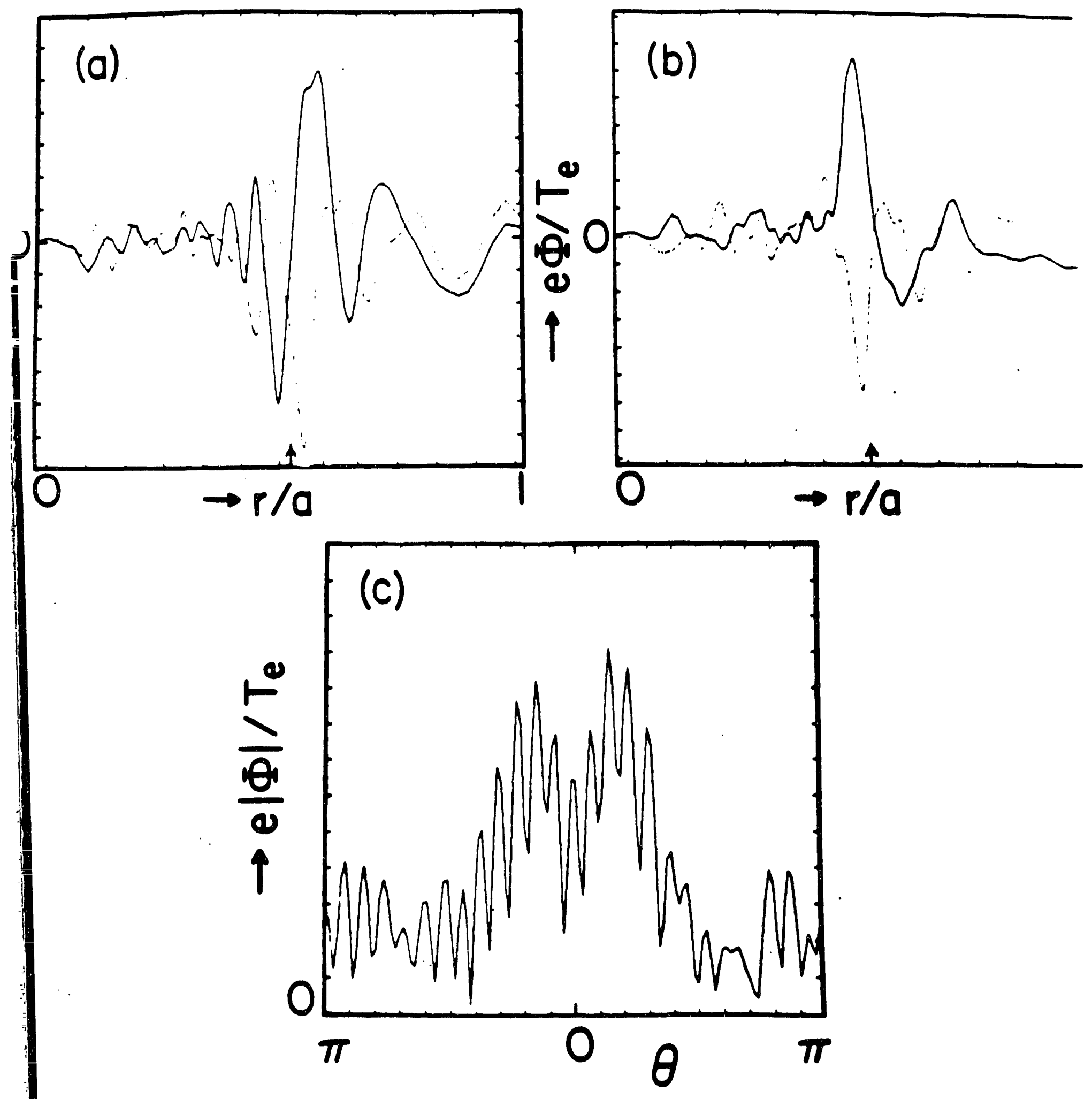

Fig. 7 

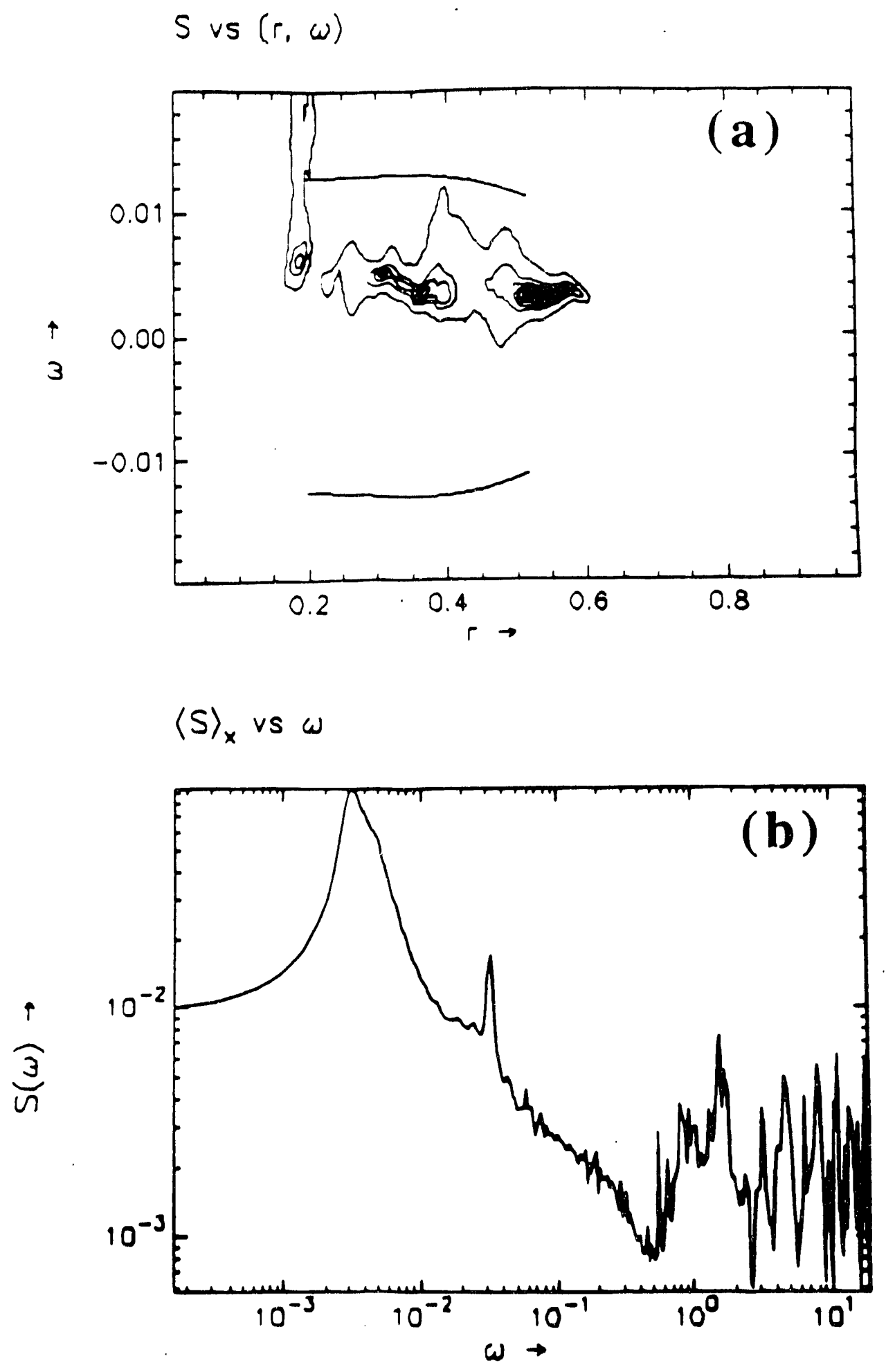

Fig. 8 


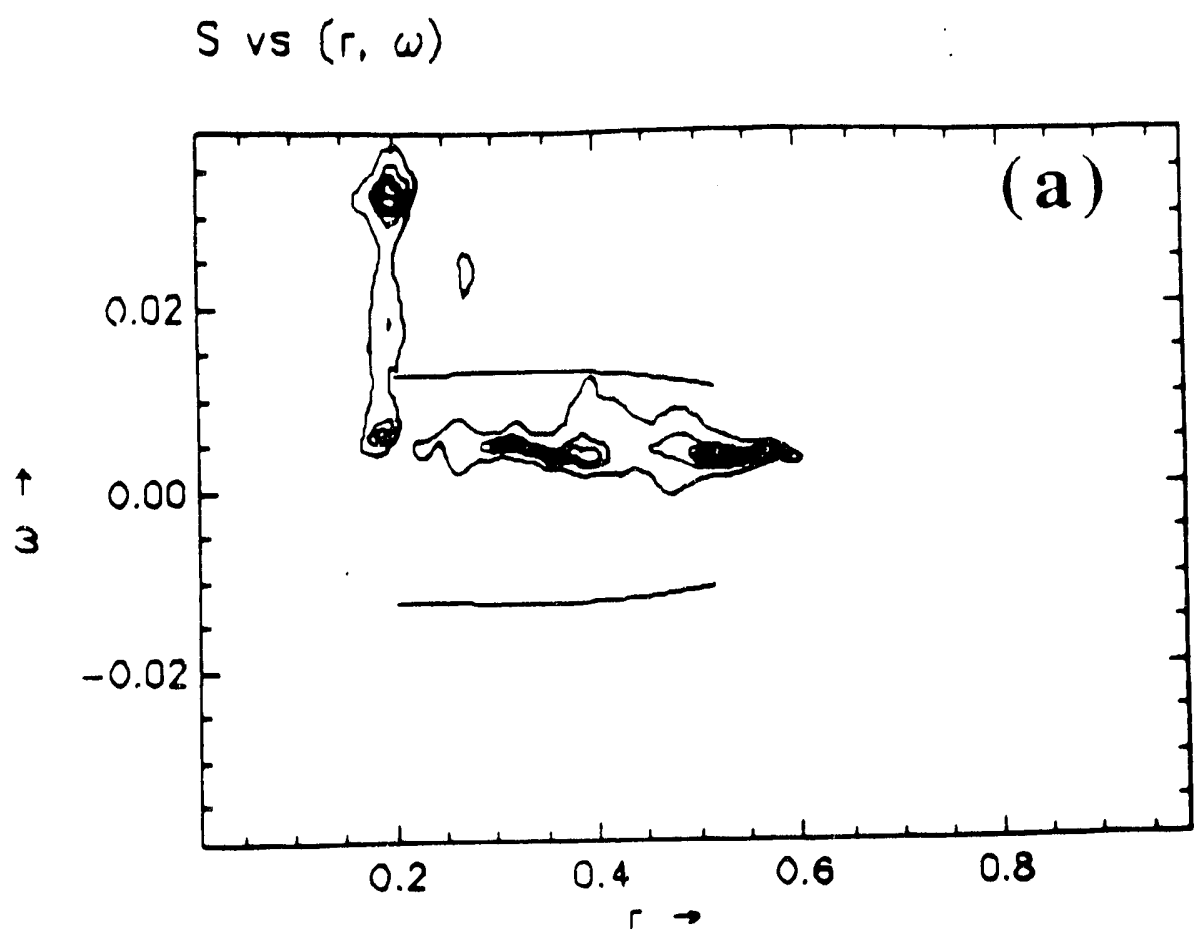

$\langle S\rangle_{x}$ vs $w$

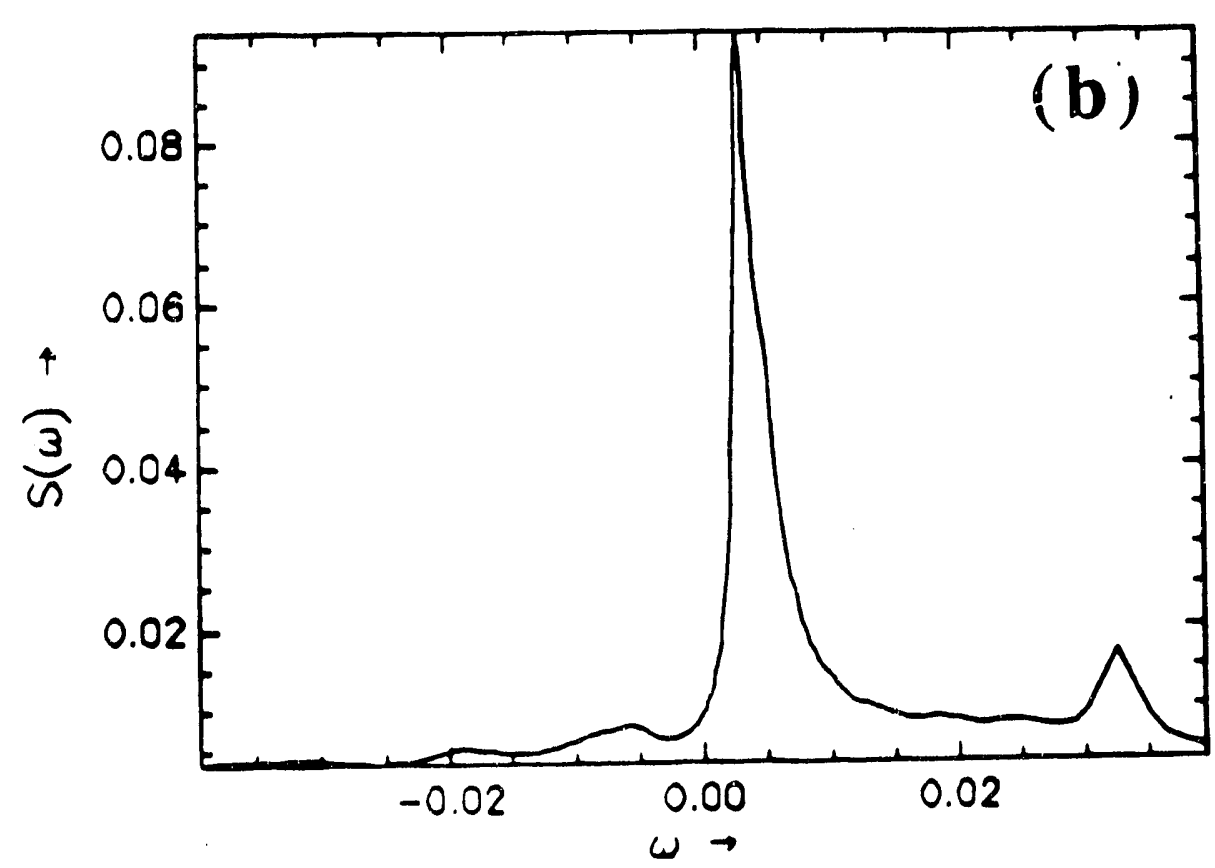

Fig. 9 

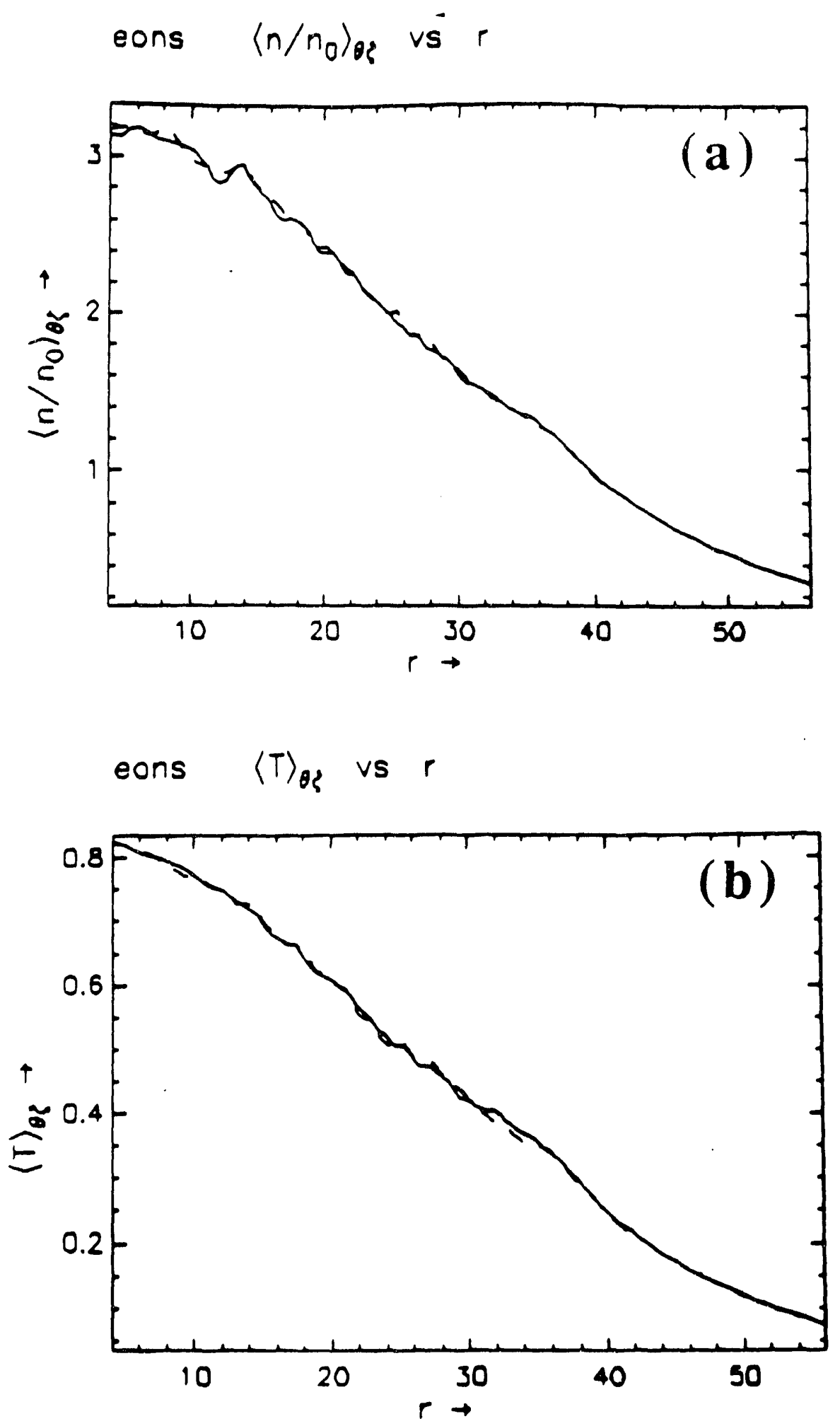

Fig. 10 
ions $\left\langle n / n_{0}\right\rangle_{\theta\}} v s r$

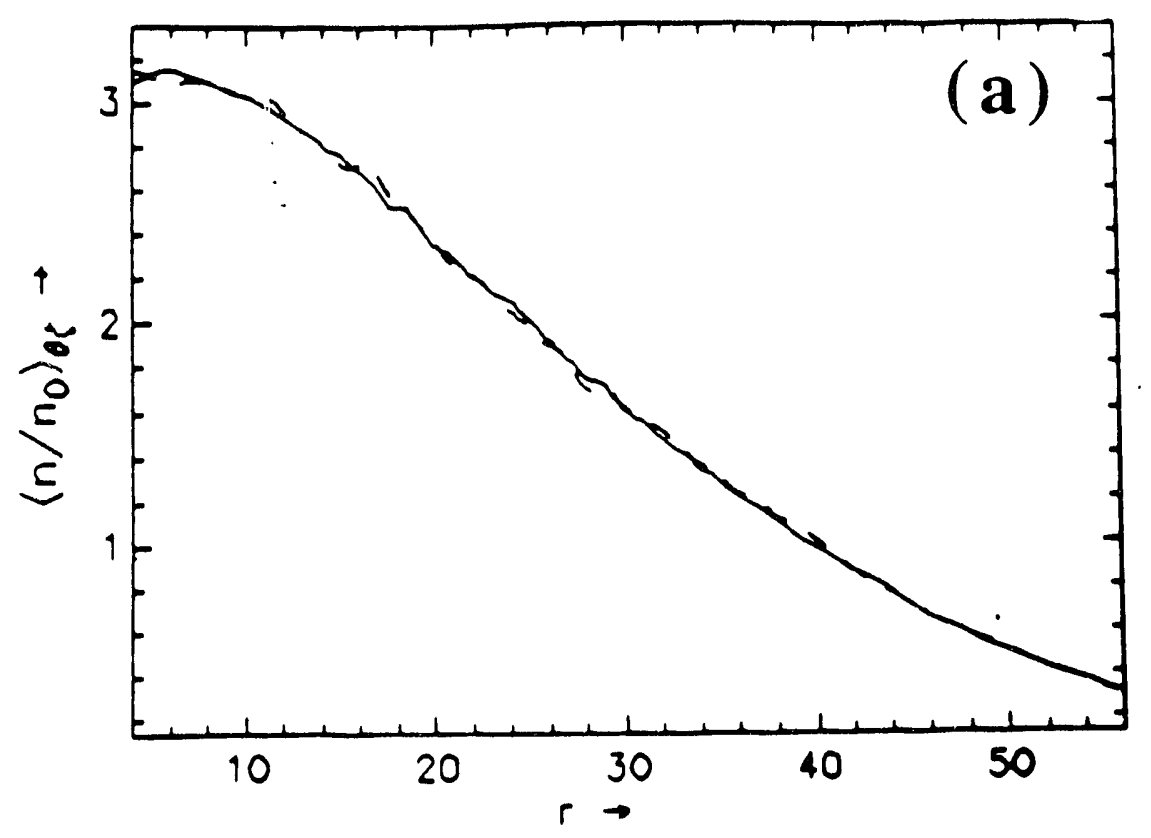

ions $\langle T\rangle_{\theta \zeta}$ vs $r$

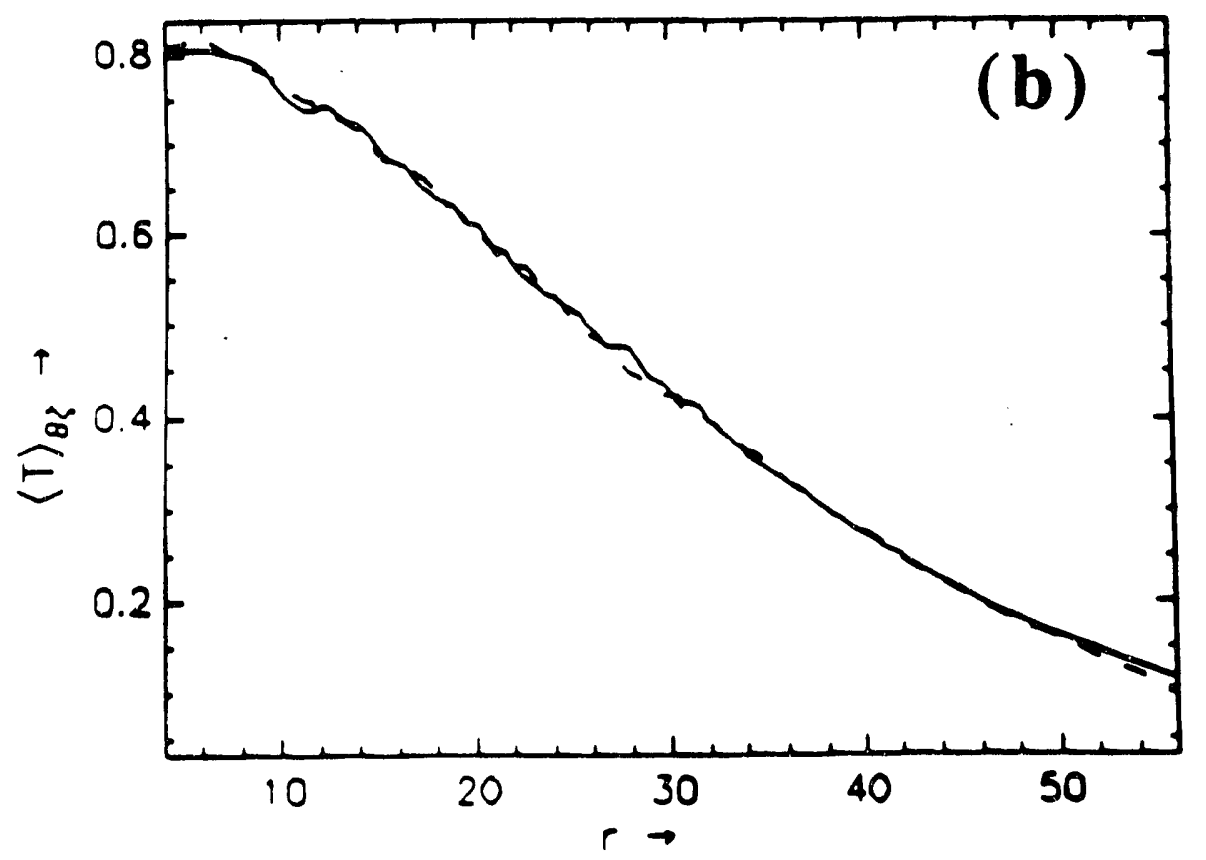

Fig. 11 


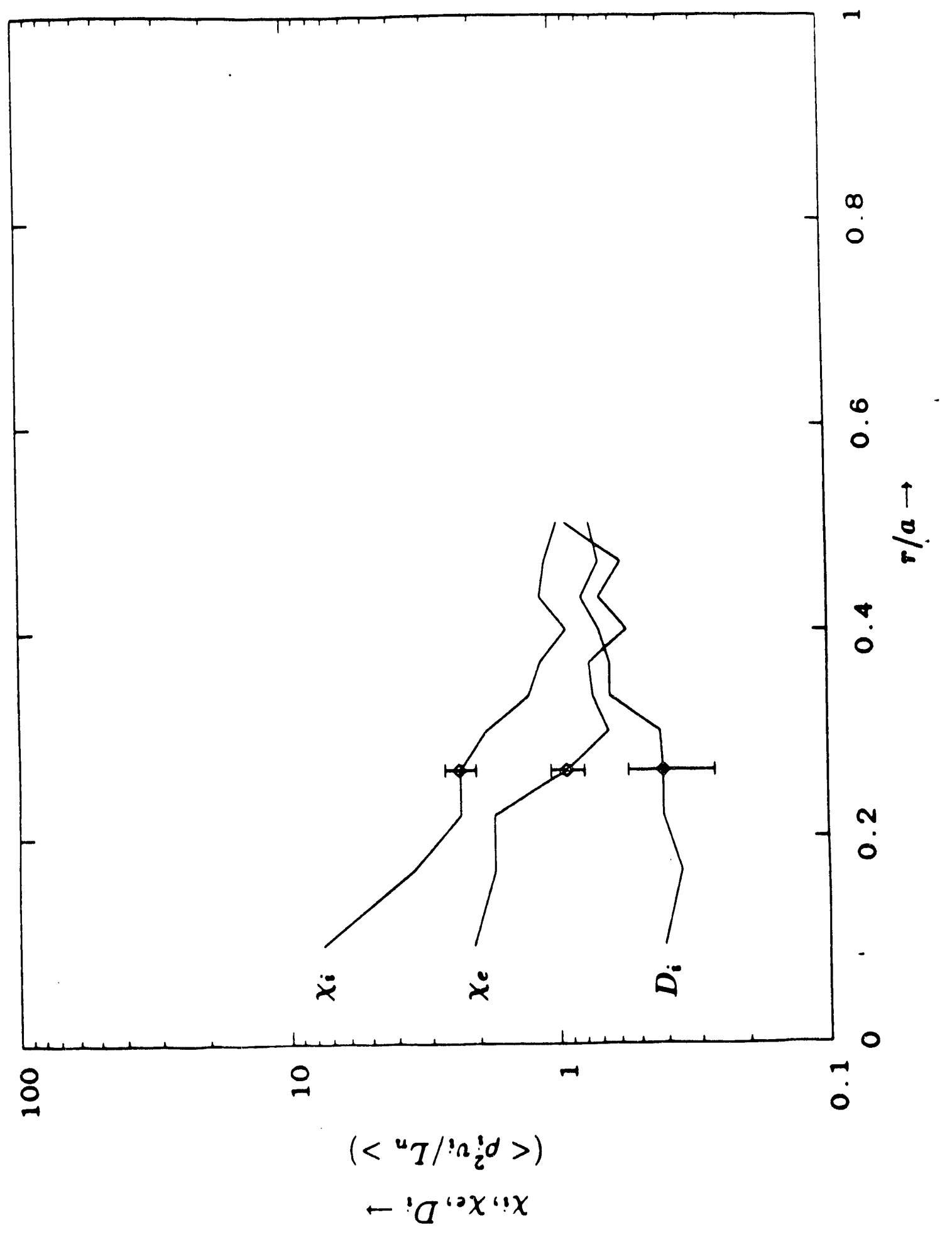

Fig. 12 

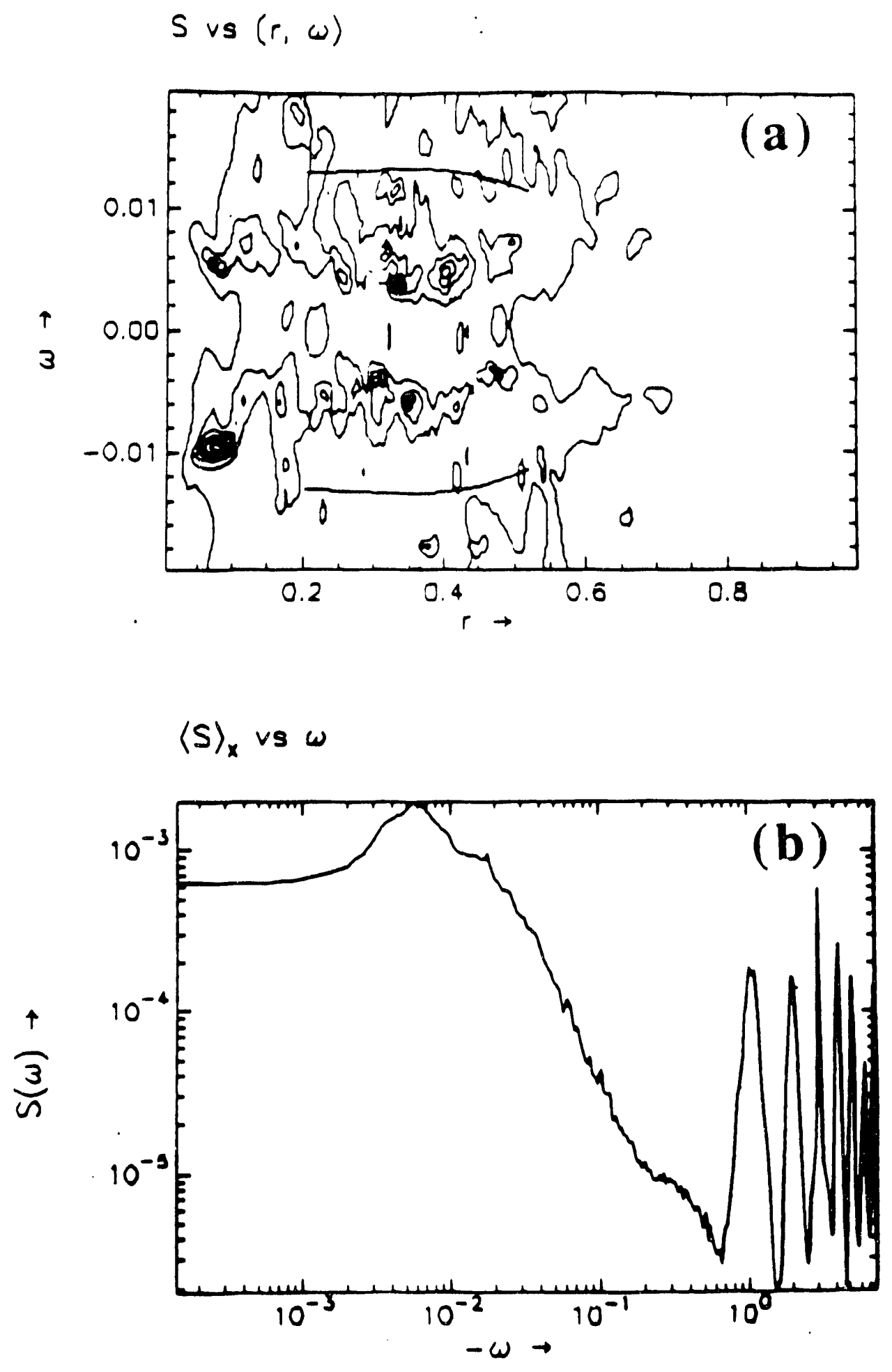

Fig. 13 
ions $\left\langle n / n_{0}\right\rangle_{\theta \zeta}$ vs $r$
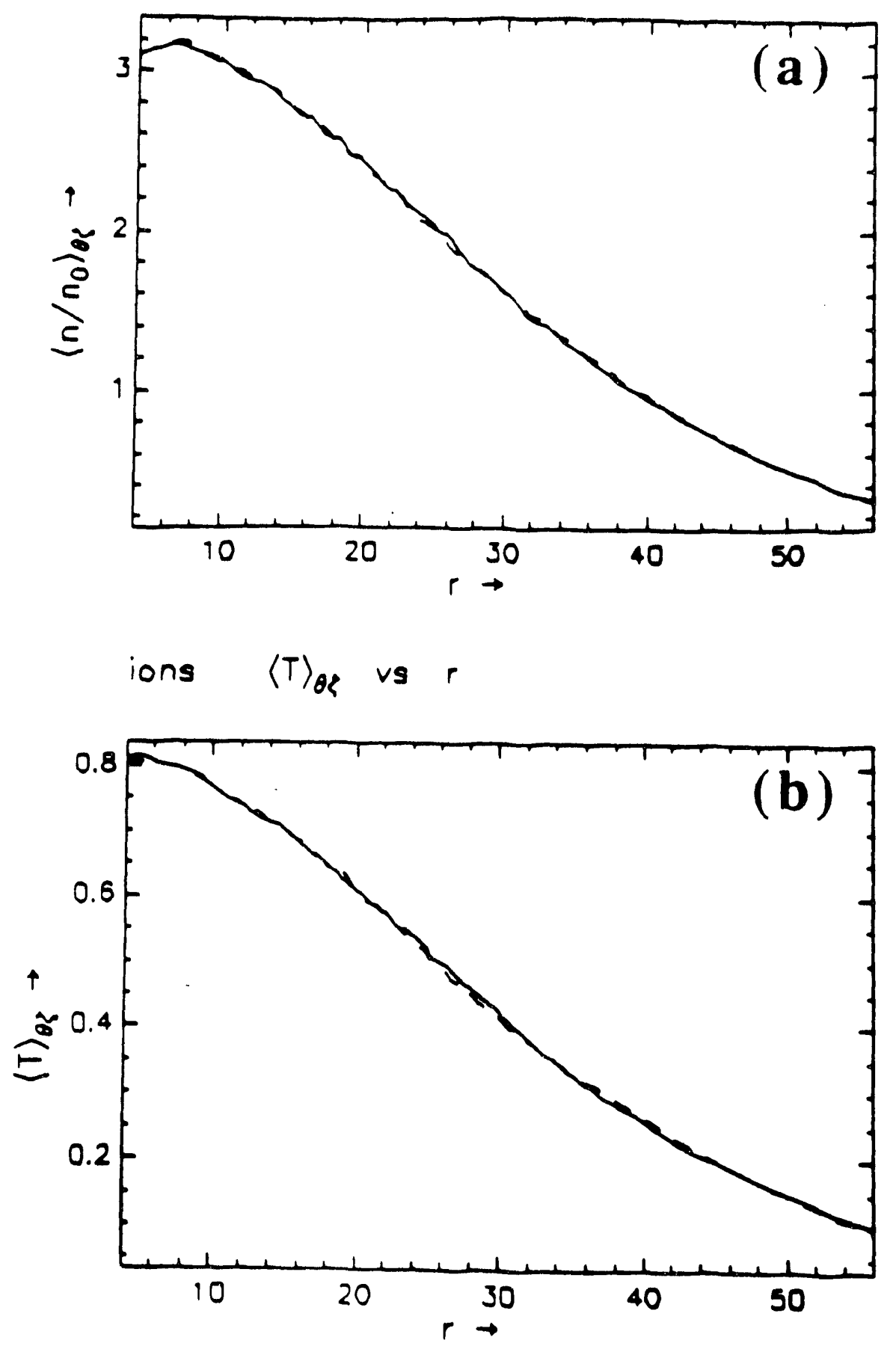

Fig. 14 


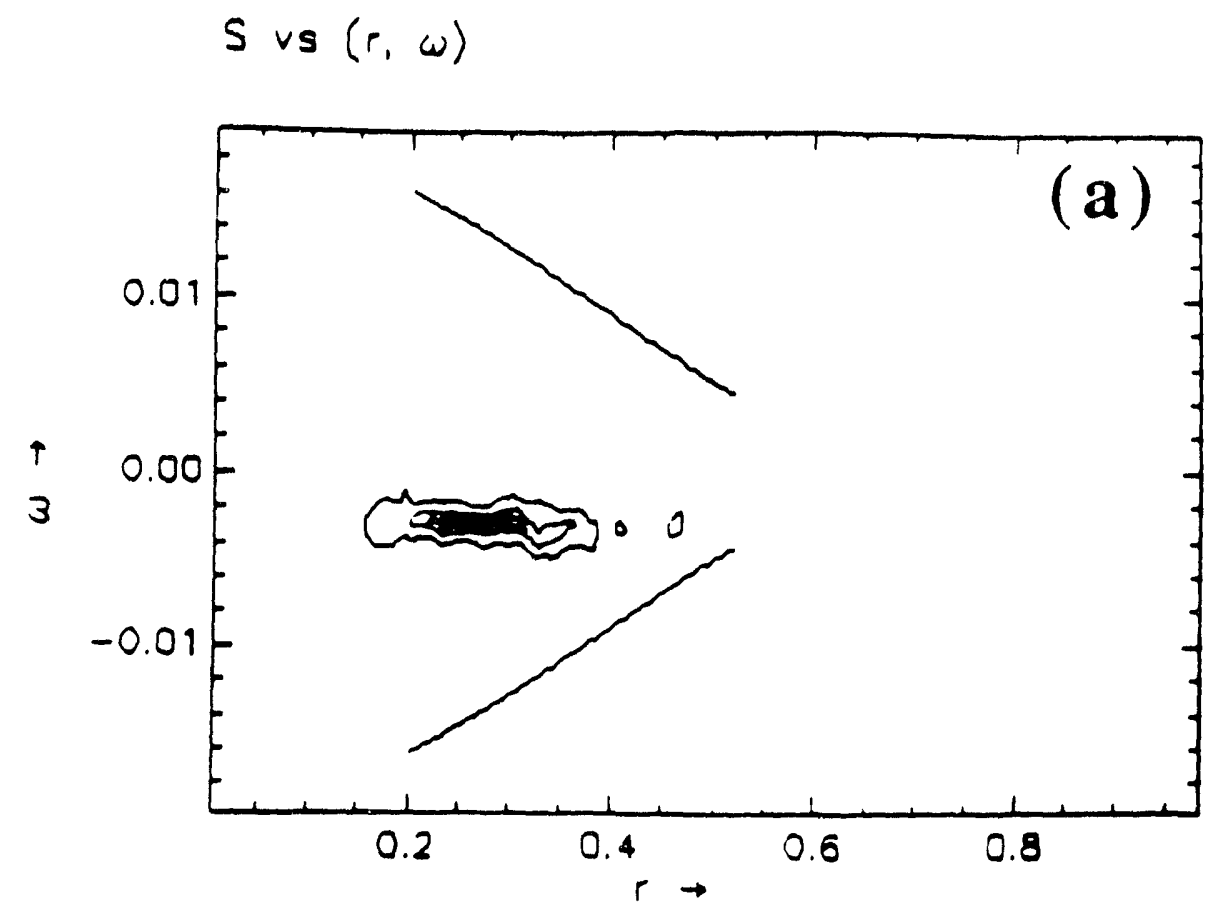

$\langle S\rangle_{x}$ vs $\omega$

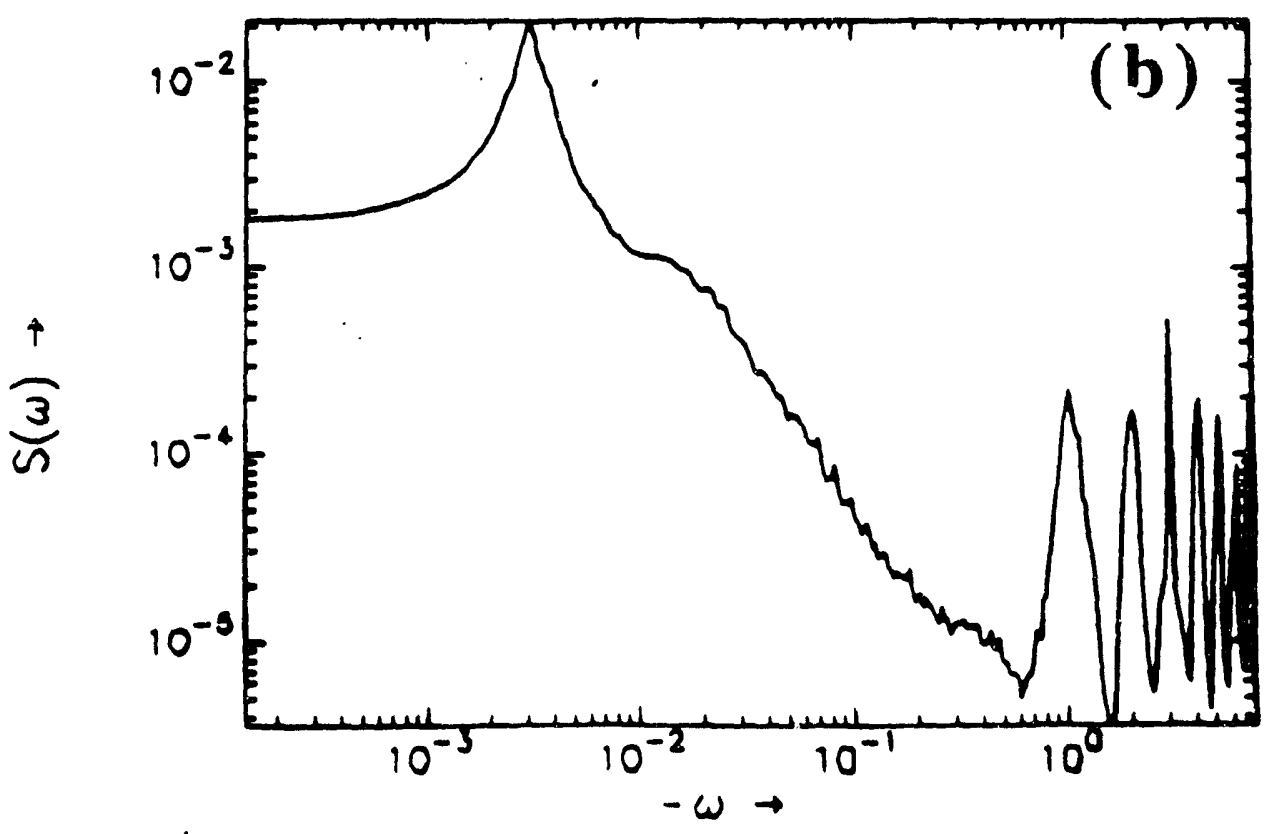

Fig. 15 

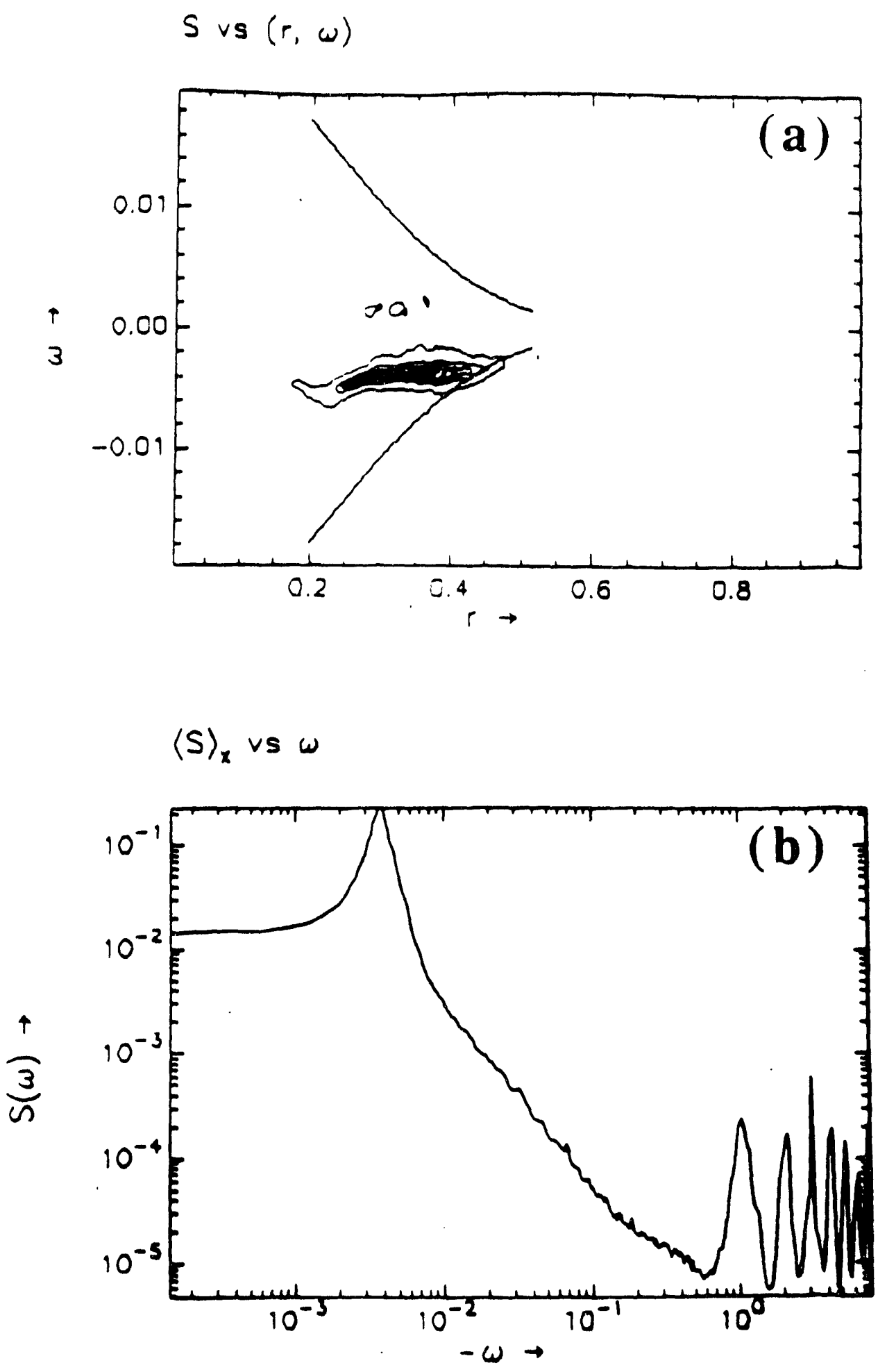

Fig. 16 

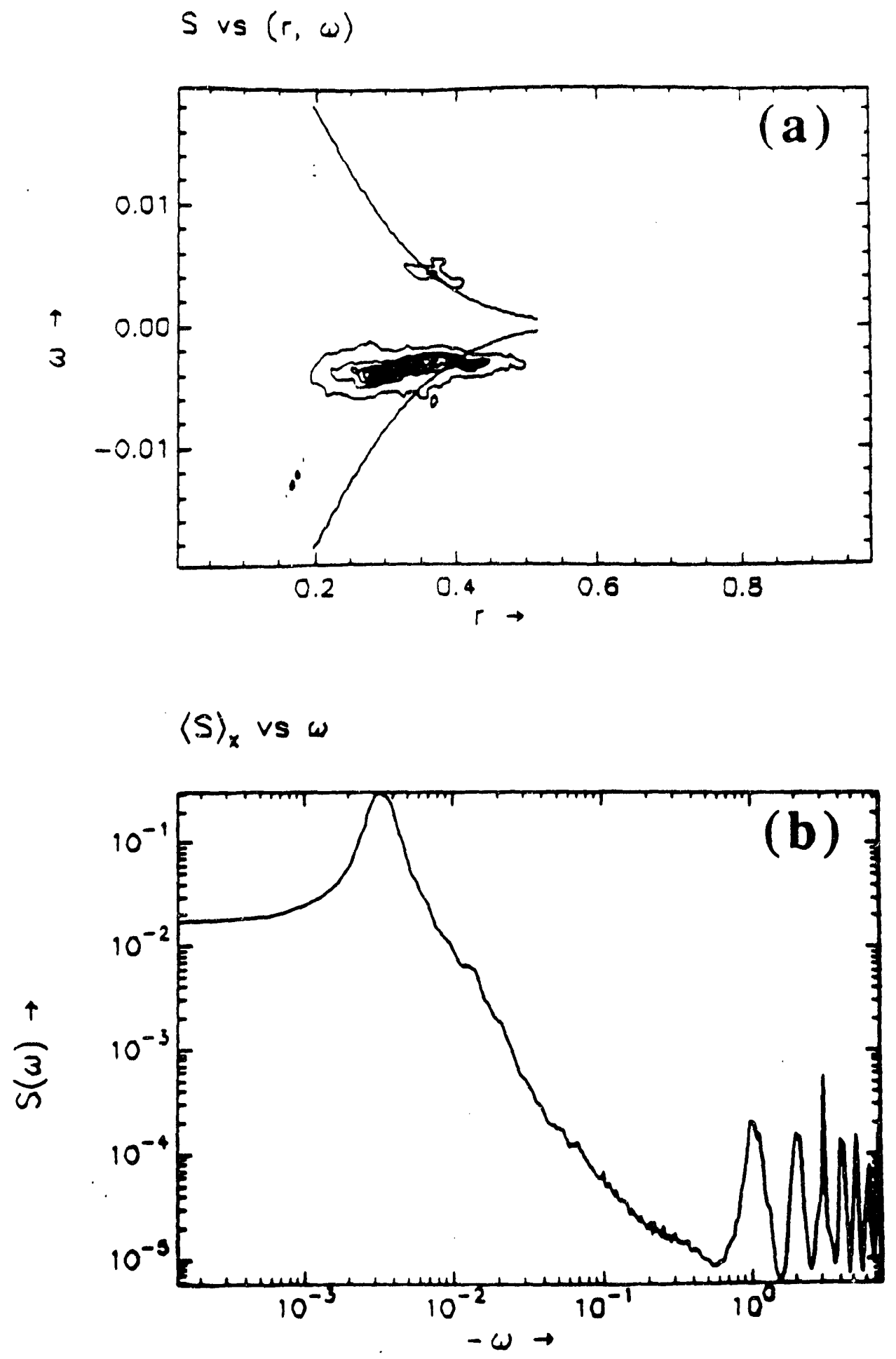

Fig. 1? 

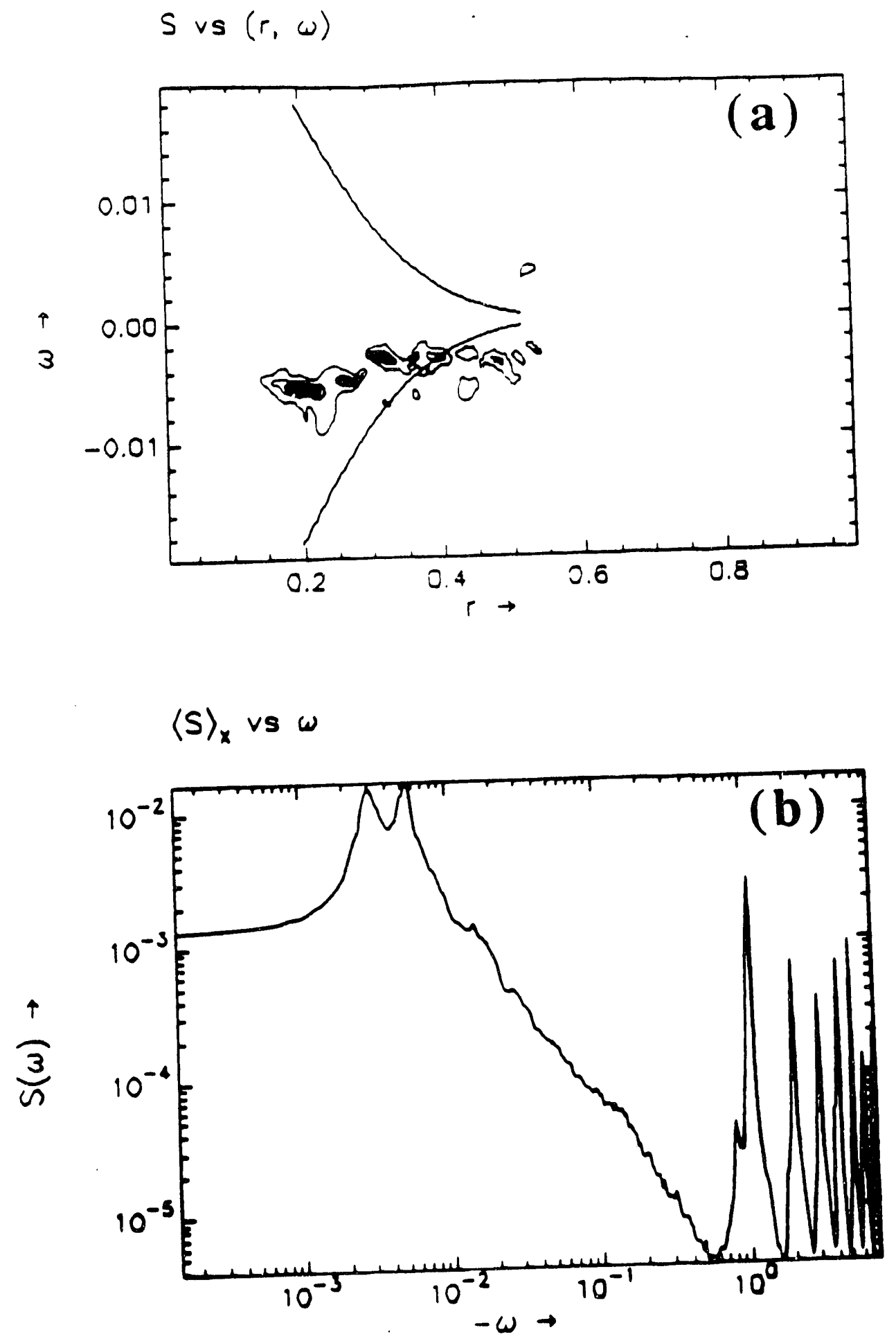

Fig. 18 


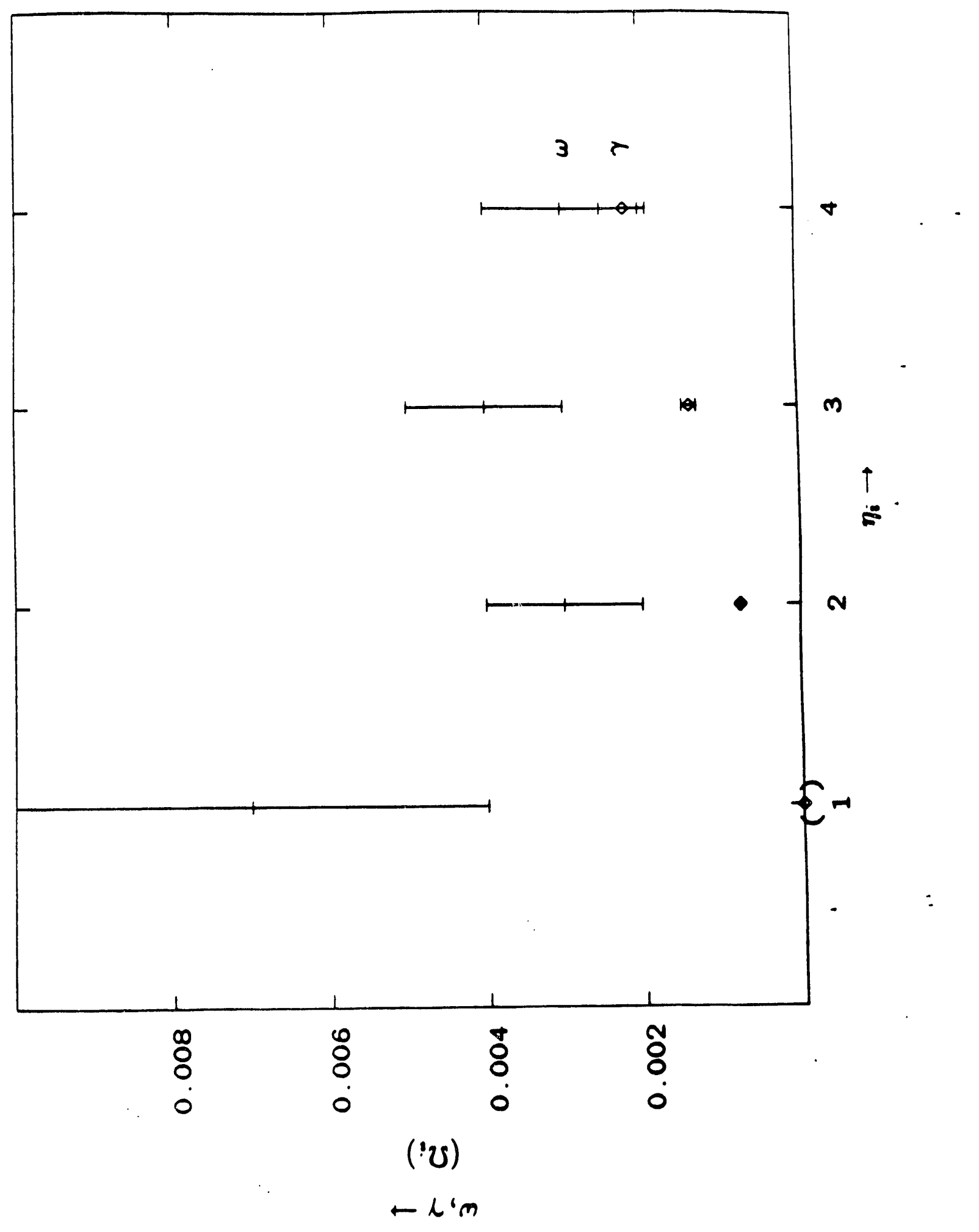

Fig. 19 

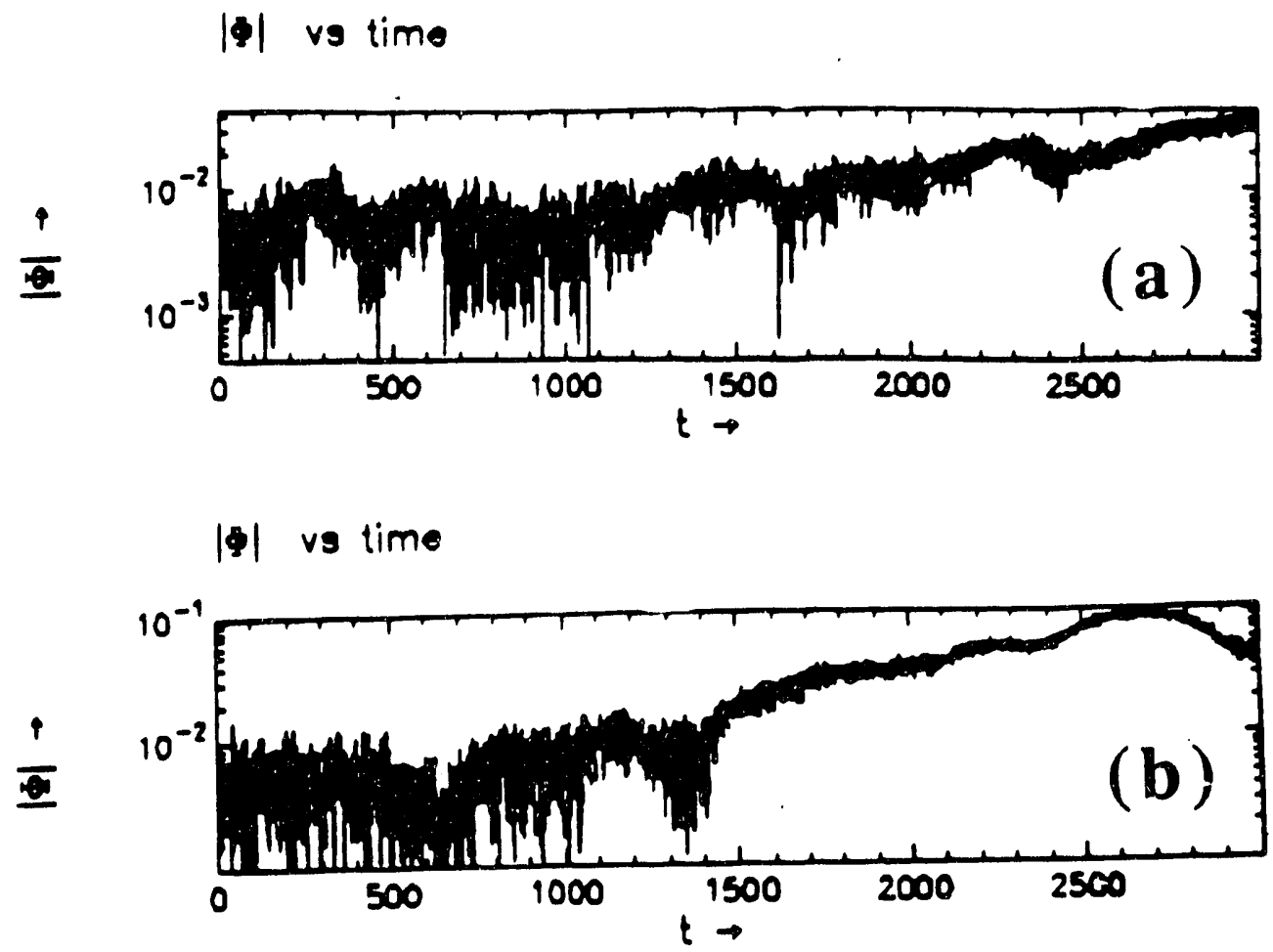

|q | vs time

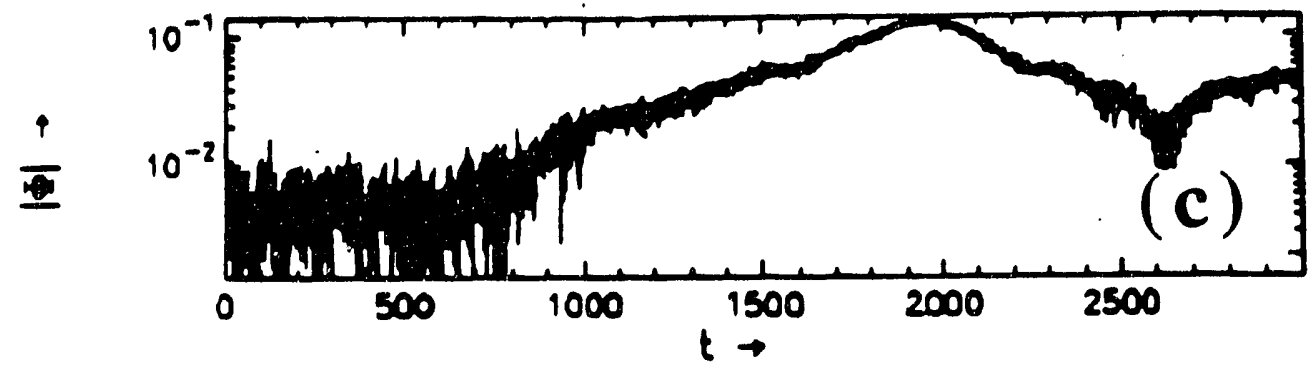

Fig. 20 

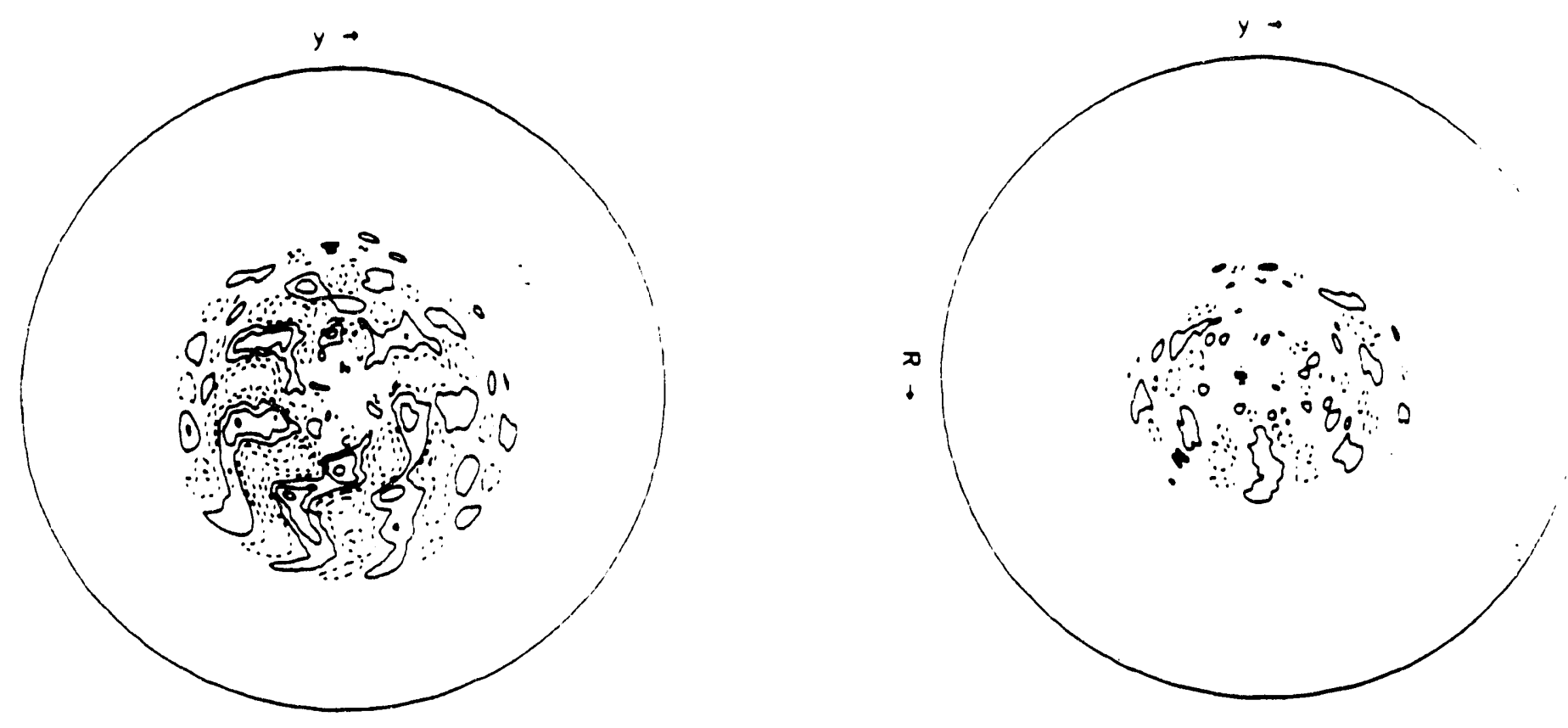

a
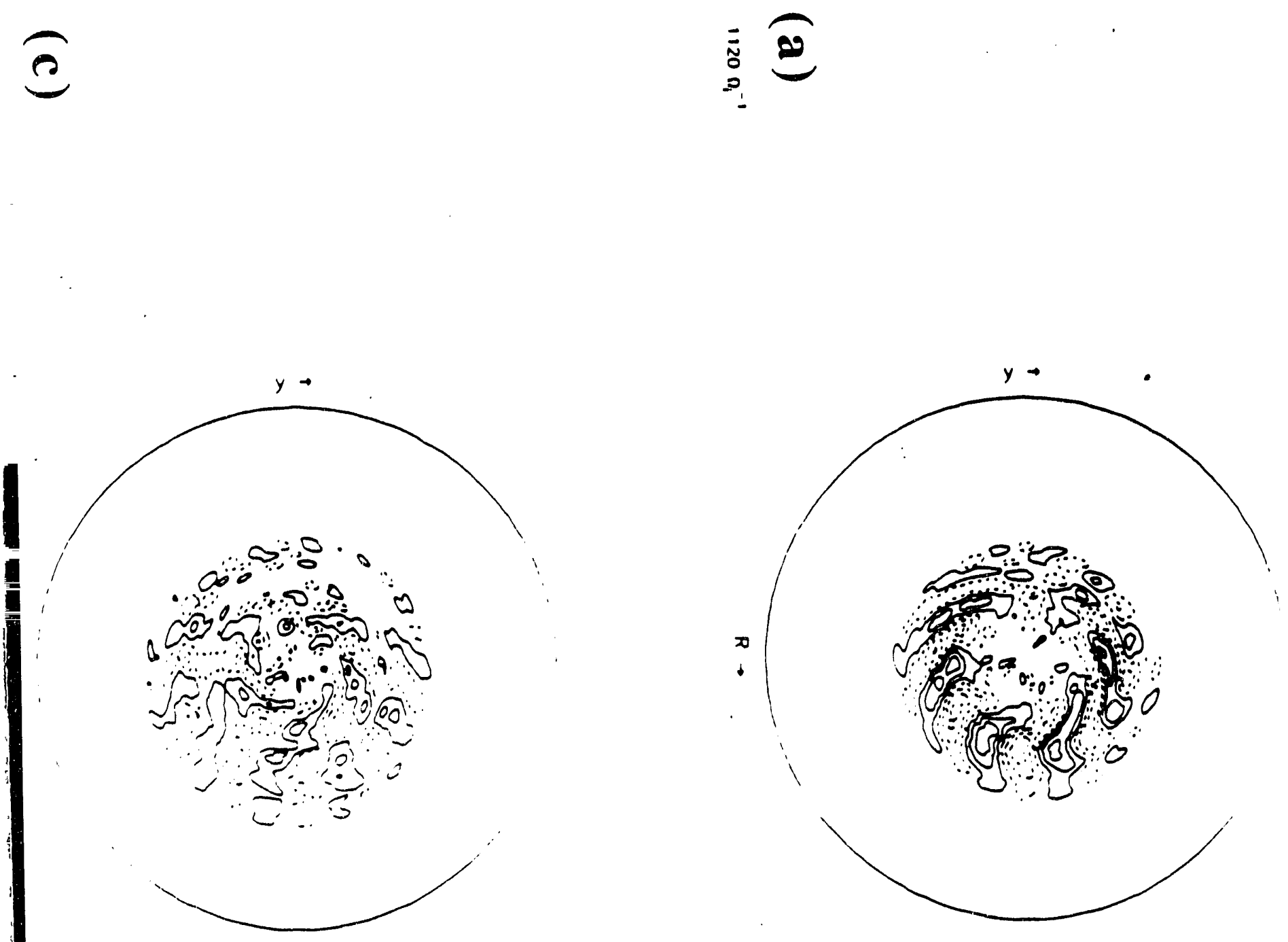

$\hat{\Xi}$

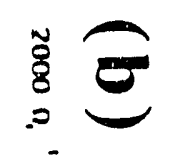

Fig. 21 
ions $\left\langle n / n_{0}\right\rangle_{\text {or }}$ vs. r

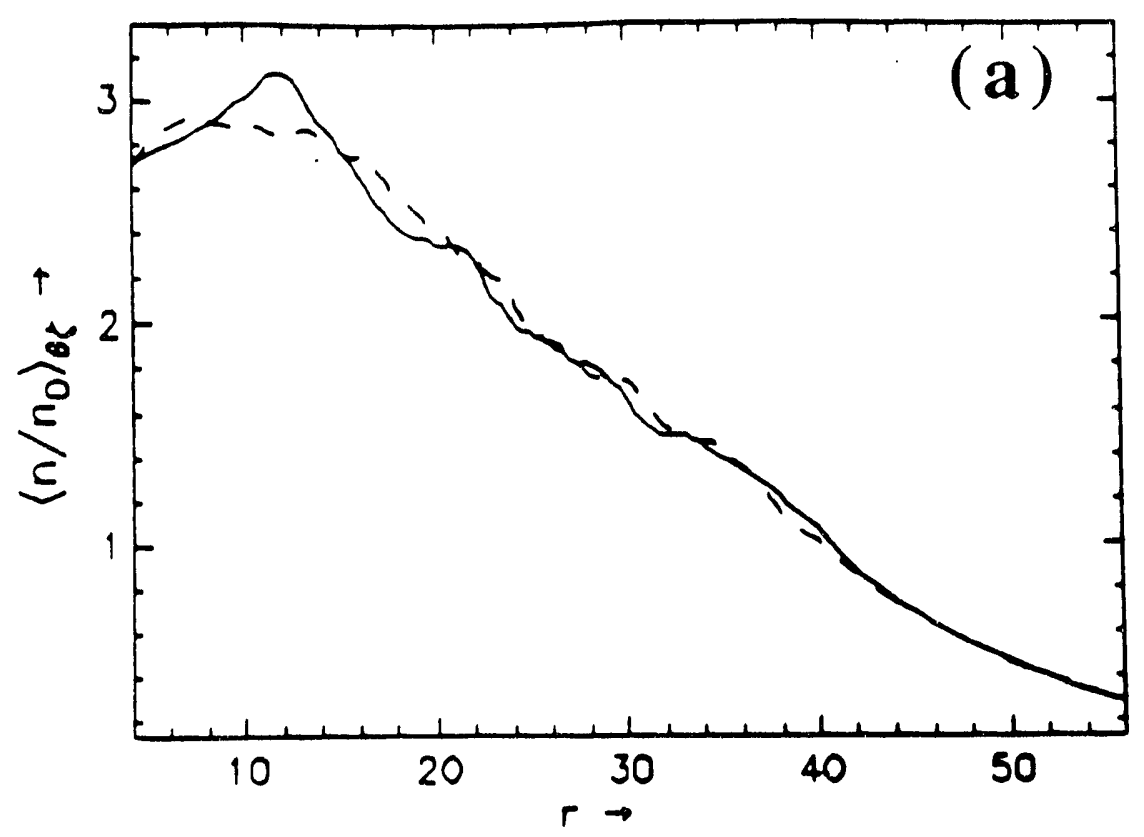

ions $\langle T\rangle_{\text {OS }}$ vS $r$

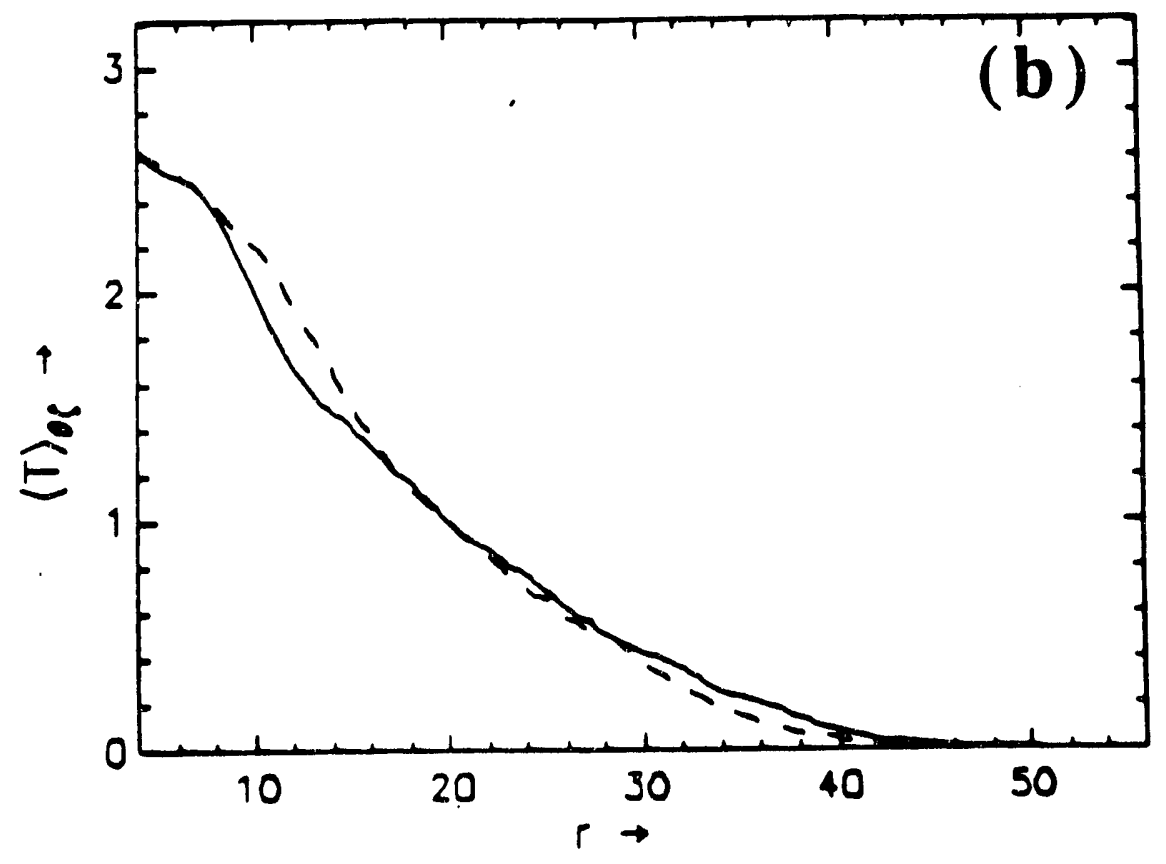

Fig. 2? 


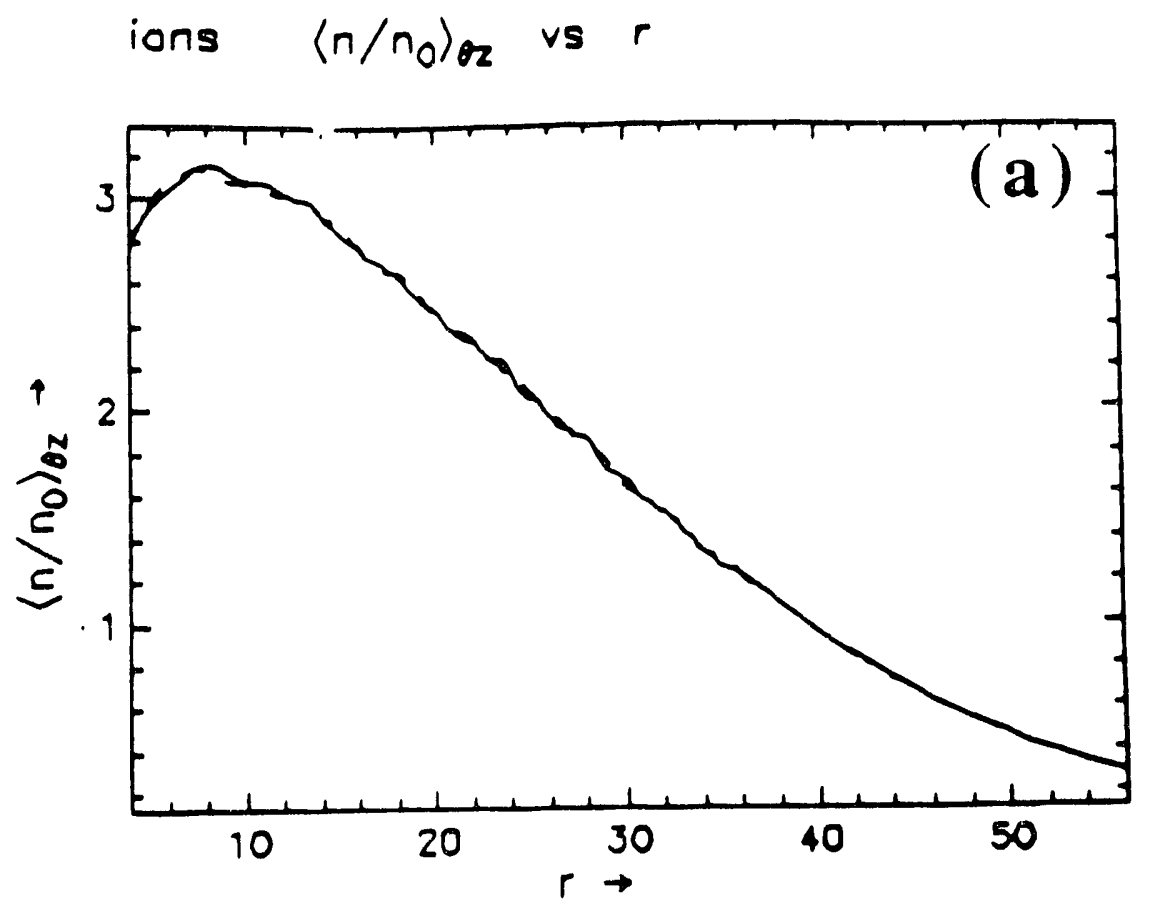

ions $\langle T\rangle_{\text {or }}$ vs $r$

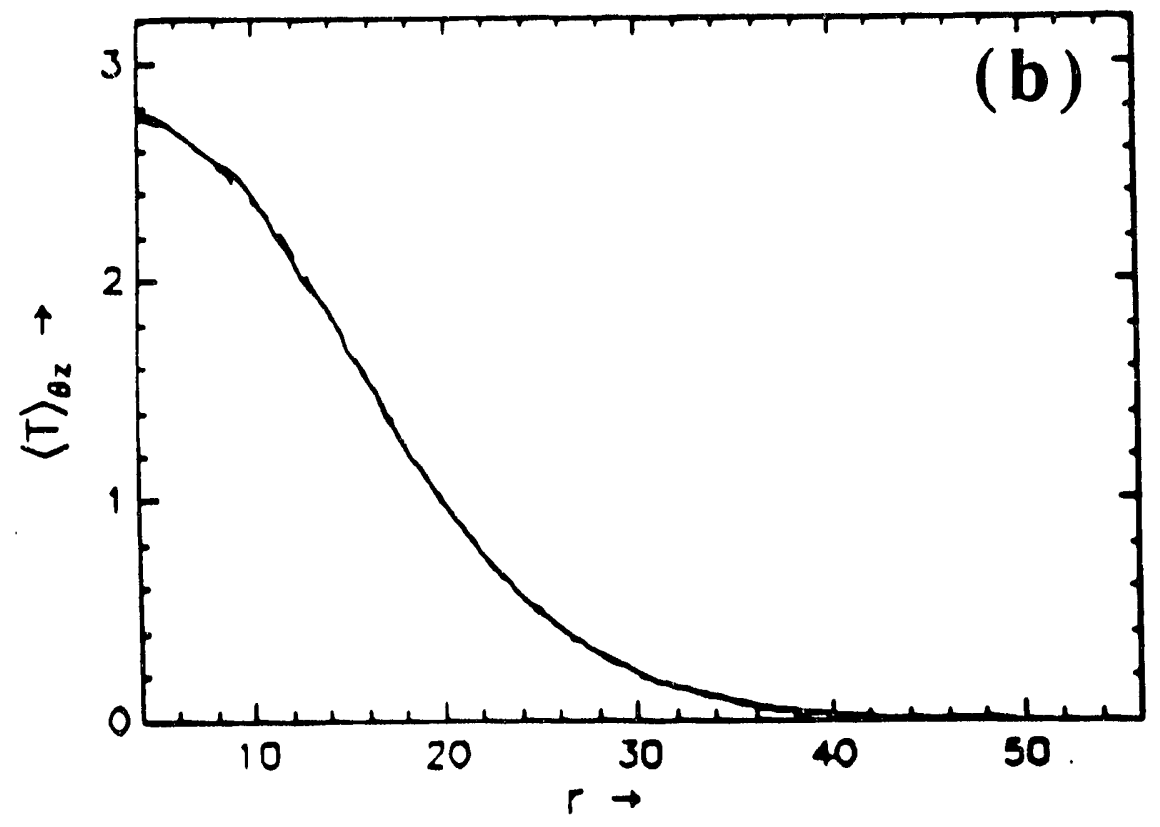

Fig. 23 


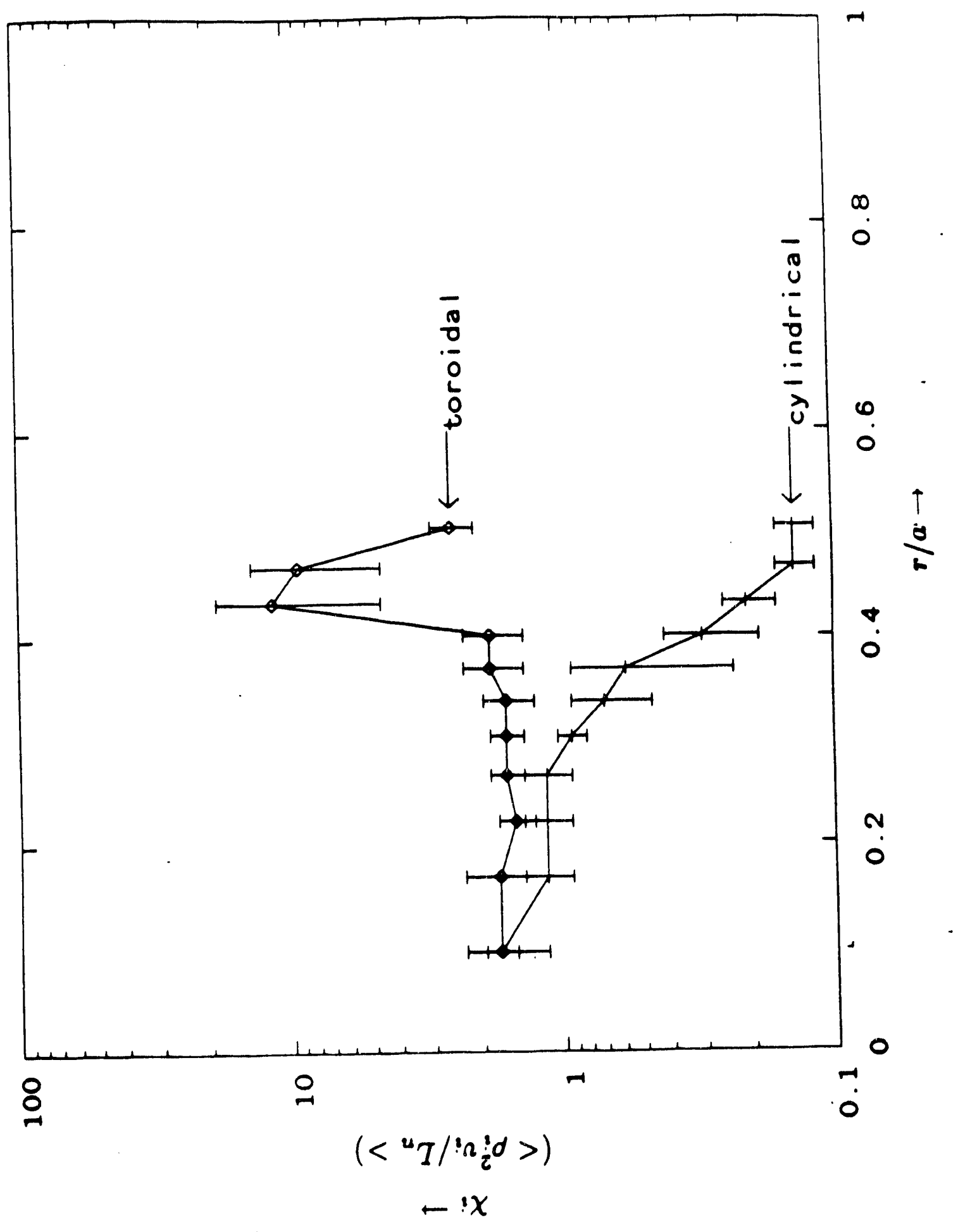

Fig. 24 


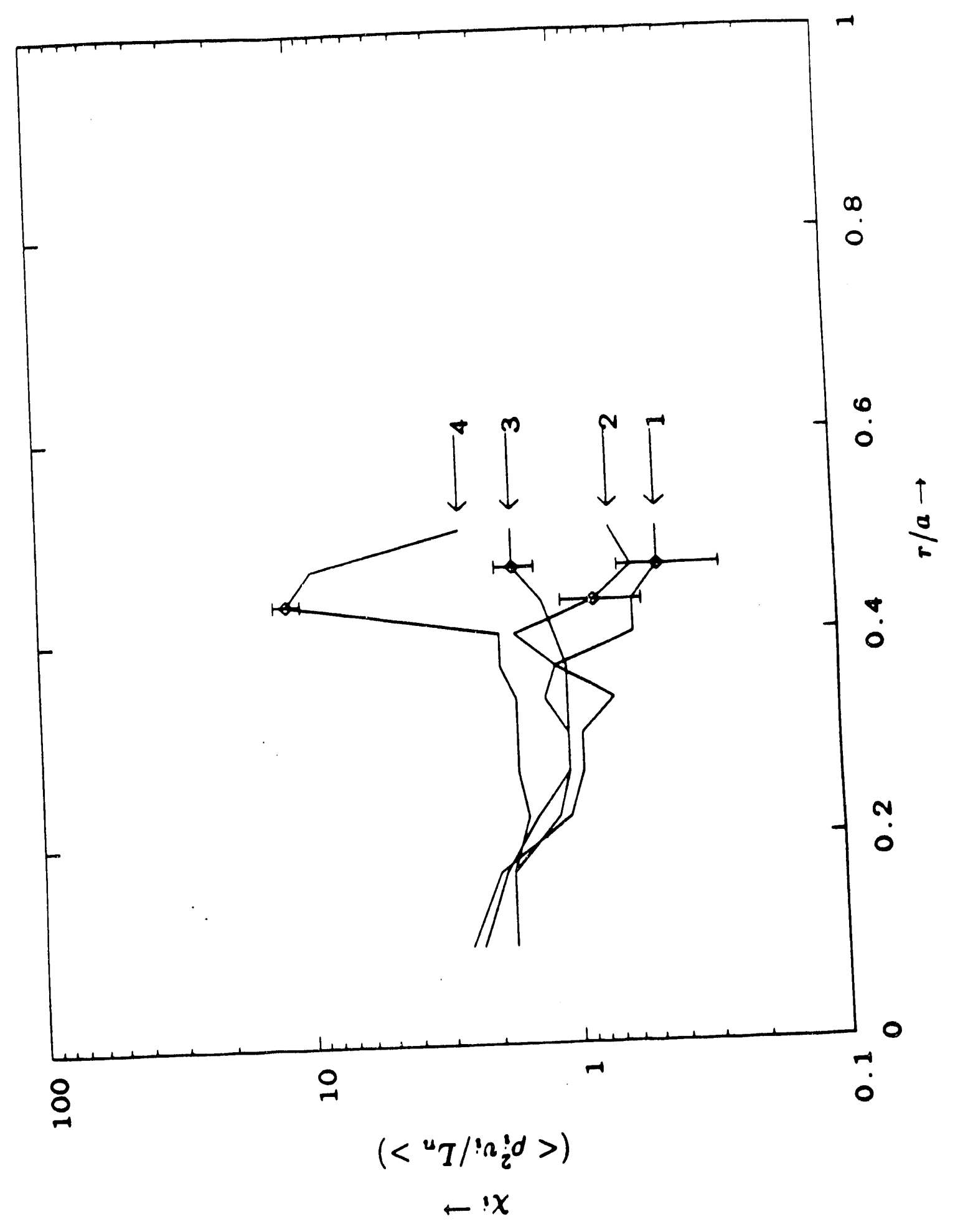

Fig. 25 


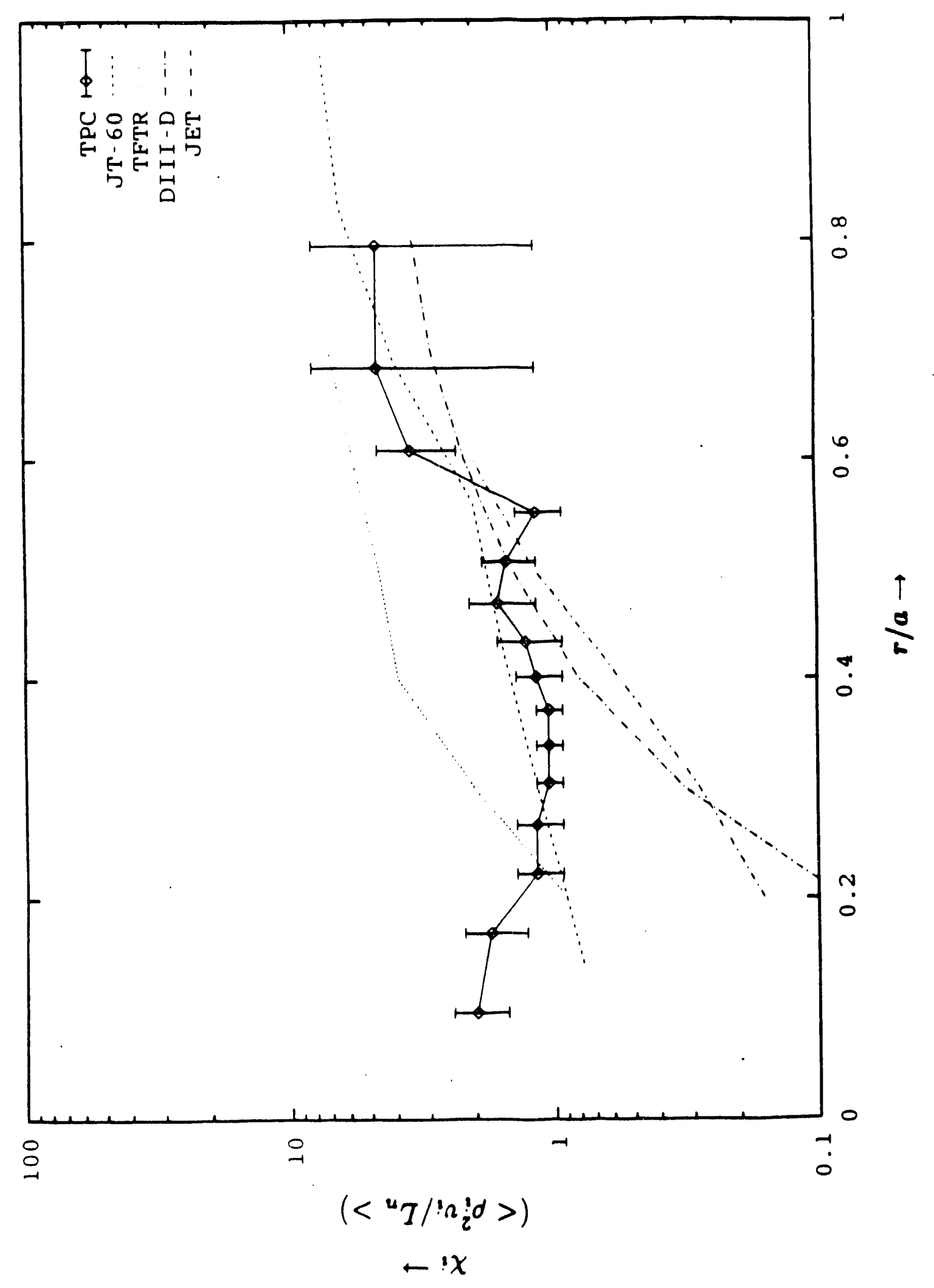

Fig. 26 


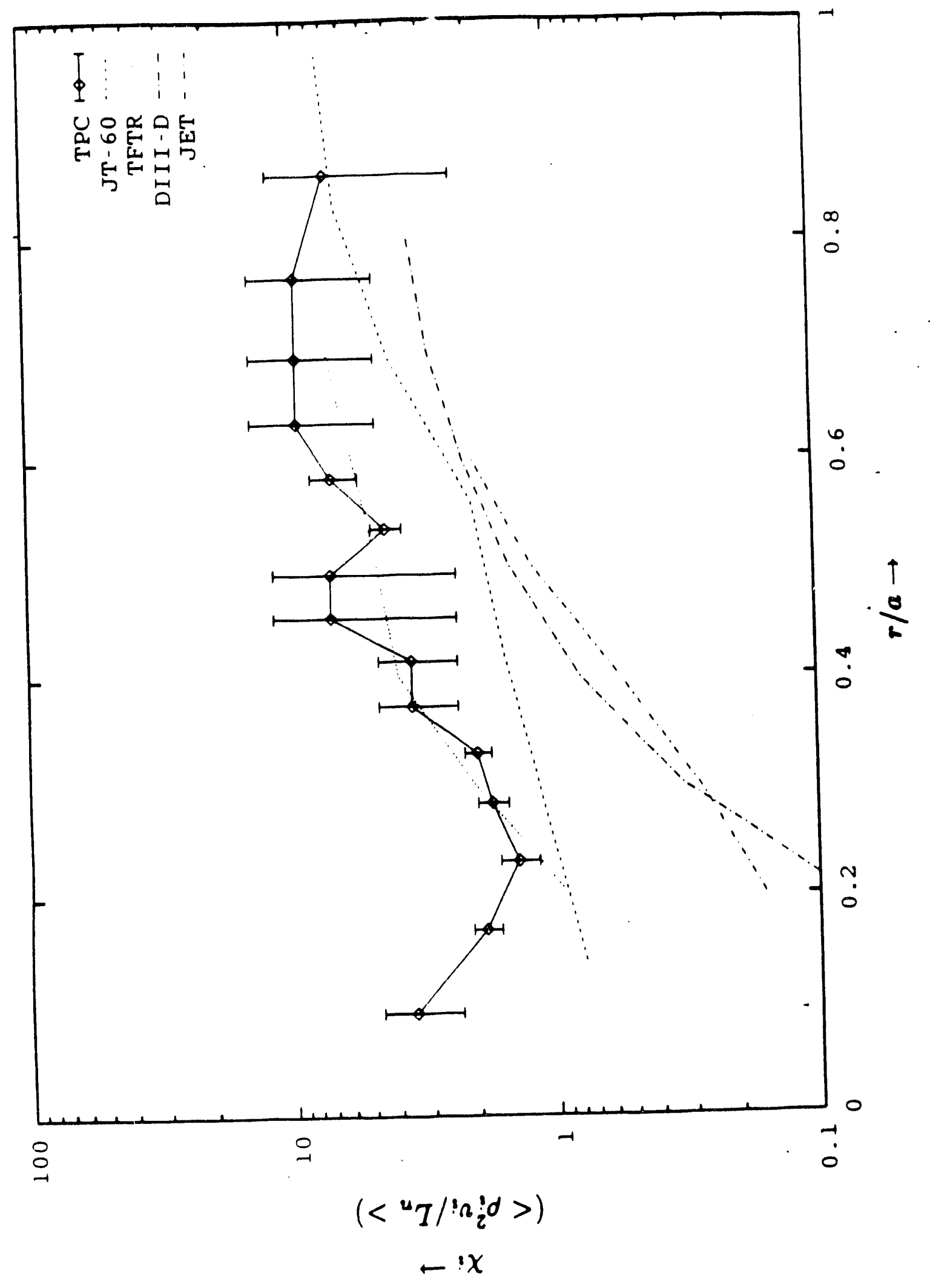

Fig. 2- 

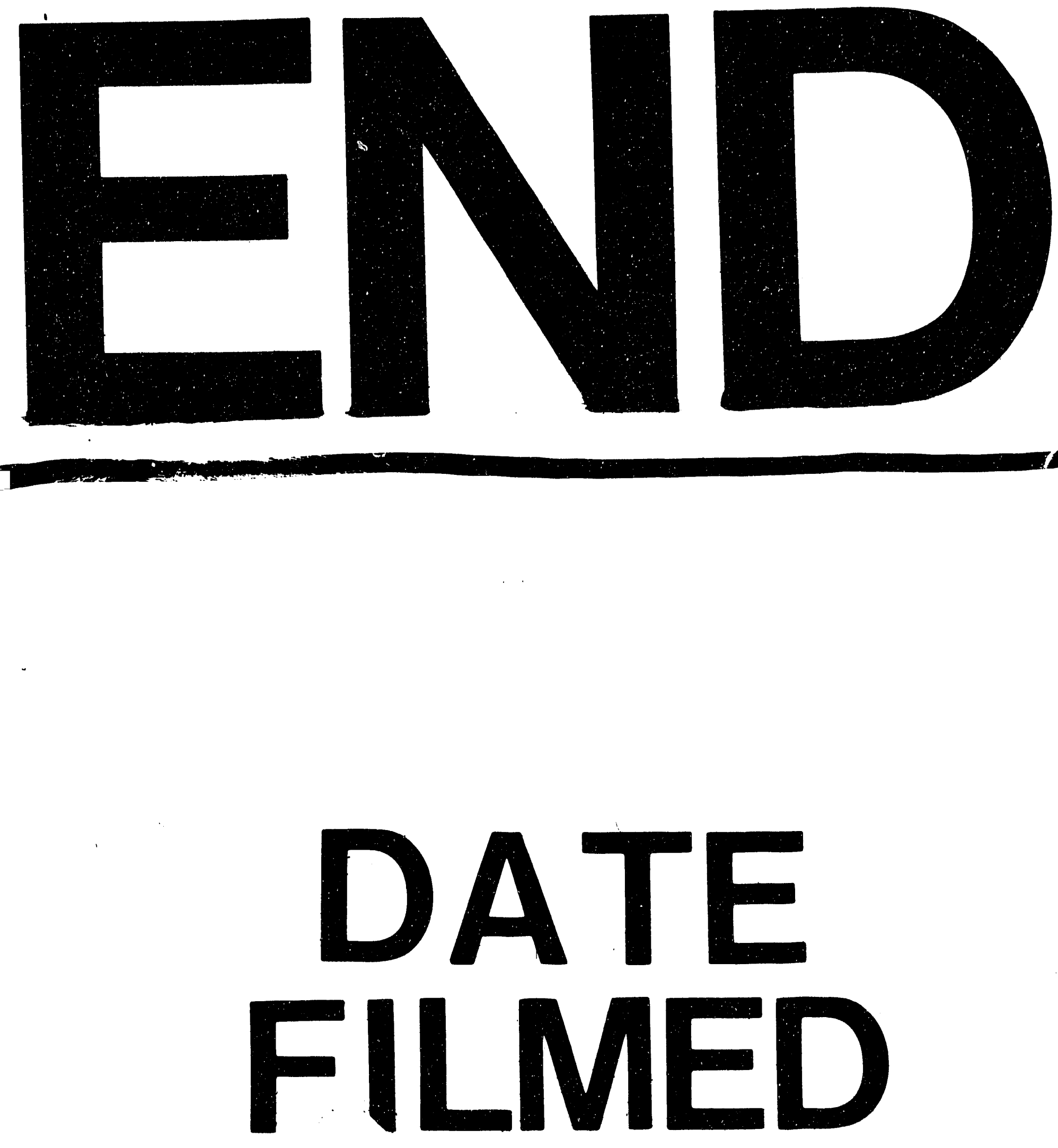

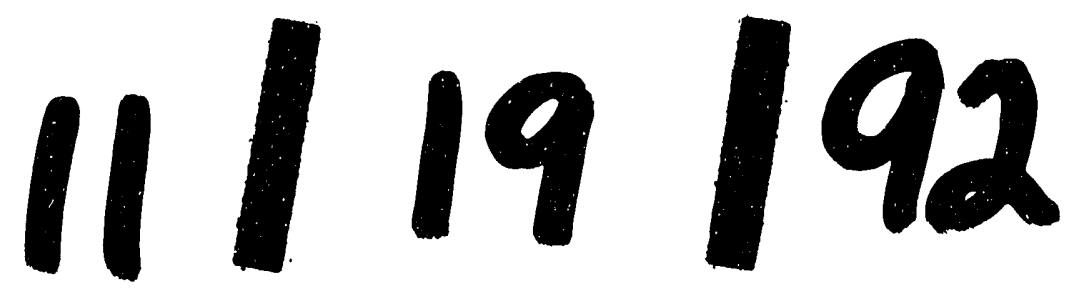


